\title{
ON THE KORTEWEG-DE VRIES EQUATION: FREQUENCIES AND INITIAL VALUE PROBLEM
}

\author{
D. Bättig, T. Kappeler And B. Mityagin
}

The Korteweg-de Vries equation (KdV)

$$
\partial_{t} v(x, t)+\partial_{x}^{3} v(x, t)-3 \partial_{x} v(x, t)^{2}=0 \quad\left(x \in S^{1}, t \in \mathbb{R}\right)
$$

is a completely integrable system with phase space $L^{2}\left(S^{1}\right)$. Although the Hamiltonian $\mathcal{H}(q):=\int_{S^{1}}\left(\frac{1}{2}\left(\partial_{x} q(x)\right)^{2}+q(x)^{3}\right) d x$ is defined only on the dense subspace $H^{1}\left(S^{1}\right)$, we prove that the frequencies $\omega_{j}=\frac{\partial \mathcal{H}}{\partial J_{j}}$ can be defined on the whole space $L^{2}\left(S^{1}\right)$, where $\left(J_{j}\right)_{j \geq 1}$ denote the action variables which are globally defined on $L^{2}\left(S^{1}\right)$. These frequencies are real analytic functionals and can be used to analyze Bourgain's weak solutions of $K d V$ with initial data in $L^{2}\left(S^{1}\right)$. The same method can be used for any equation in the $K d V$-hierarchy.

\section{Introduction and summary of the results.}

It is well known that the Korteweg-de Vries equation $(K d V)$ on the circle

$$
\partial_{t} v(x, t)=-\partial_{x}^{3} v(x, t)+6 v(x, t) \partial_{x} v(x, t)
$$

can be viewed as a completely integrable Hamiltonian system of infinite dimension. We choose as its phase space $L^{2}\left(S^{1}\right)=L^{2}\left(S^{1} ; \mathbb{R}\right)$ where $S^{1}$ is the circle of length 1 . The Poisson structure is the one proposed by Gardner,

$$
\{F, G\}=\int_{S^{1}} \frac{\partial F}{\partial q(x)} \frac{d}{d x} \frac{\partial G}{\partial q(x)} d x
$$

where $F, G$ are $C^{1}$ - functionals on $L^{2}\left(S^{1}\right)$, and $\frac{\partial F}{\partial q(x)}$ denotes the $L^{2}$-gradient of the functional $F$. The Hamiltonian $\mathcal{H}$ corresponding to $K d V$ is then given by

$$
\mathcal{H}(q):=\int_{S^{1}}\left(\frac{1}{2}\left(\partial_{x} q\right)^{2}+q^{3}\right) d x
$$


Note that the Poisson structure (1.2) is degenerate and admits the average as a Casimir function

$$
[q]=\int_{S^{1}} q(x) d x .
$$

Moreover, the Poisson structure is regular and induces a trivial foliation whose leaves are given by

$$
L_{c}^{2}\left(S^{1}\right)=\left\{q \in L^{2}\left(S^{1}\right) ;[q]=c\right\} .
$$

Consider the leaf $L_{0}^{2}\left(S^{1}\right)$ and denote by $\omega_{G}$ the symplectic structure on $L_{0}^{2}\left(S^{1}\right)$ induced by the Poisson structure (1.2).

In previous work $[\mathbf{K a}],[\mathbf{B B G K}]$ the phase space $\left(L_{0}^{2}\left(S^{1}\right), \omega_{G}\right)$ was analyzed and it was proved that $K d V$ admits globally defined (generalized) action-angle variables. Recall that for a finite dimensional, completely integrable Hamiltonian system action-angle variables linearize the flow and, therefore, solutions can be found by quadrature. When trying to apply this procedure to find $L^{2}$-solutions of $K d V$ one notices that the Hamiltonian $\mathcal{H}$ is only defined on the dense subspace $H^{1}\left(S^{1}\right)$ and so are the frequencies $\omega_{j}=\frac{\partial \mathcal{H}}{\partial J_{j}}$, given by the partial derivatives of $\mathcal{H}$ with respect to the action variables $J_{j}$. In Section 3, using auxilary results proved in Section 2, we show the following main result of this paper:

Theorem 1. Let $q_{0} \in L_{0}^{2}\left(S^{1}\right)$. Then there exists a neighborhood $U_{q_{0}}$ of $q_{0}$ in $L_{0}^{2}\left(S^{1} ; \mathbb{C}\right)$ so that the frequencies are defined and analytic on $U_{q_{0}}$. Moreover, they satisfy, uniformly on $U_{q_{0}}$,

$$
\omega_{j}=(2 \pi j)^{3}+o(1)
$$

Our starting point of the proof of Theorem 1 is the Its-Matveev formula which, at least for finite gap potentials, provides a formula for the frequencies $\omega_{j}(j \geq 1)$ in terms of periods of Abelian differentials of the second kind on the hyperelliptic Riemann surface $\Sigma_{q}$, associated to the periodic spectrum of the Schrödinger operator $-\frac{d^{2}}{d x^{2}}+q$ where $q$ is the initial data of (1.1) (cf. e.g. [DKN], [FFM], [MT1, 2]). Using Riemann bilinear relations (cf e.g. $[\mathbf{E M}])$ one sees that the frequencies $\omega_{j}(j \geq 1)$ can be expressed in terms of the zeroes of a conveniently normalized basis of holomorphic differentials on $\Sigma_{q}$. Our strategy for the proof of Theorem 1 is to show that for elements $q$ merely in $L^{2}\left(S^{1}\right)$, such a basis of holomorphic differentials still exists with the property that their zeroes are real analytic functions of $q$ and that these zeroes can be rather precisely located (cf. Section 2 and Appendix).

As an application of Theorem 1 we investigate the initial value problem for $\mathrm{KdV}$ on the circle. Denote by $\Omega: L_{0}^{2}\left(S^{1}\right) \rightarrow \ell_{1 / 2}^{2}\left(\mathbb{R}^{2}\right)$ the symplectomorphism 
constructed in $[\mathrm{BBGK}]$ and by $\left(J_{j}, \alpha_{j}\right)_{j \geq 1}$ the symplectic polar coordinates in $\ell_{1 / 2}^{2}\left(\mathbb{R}^{2}\right)$,

$$
\Omega(q)=(x, y)=\left(x_{j}, y_{j}\right)_{j \geq 1}=\left(\sqrt{2 J_{j}} \cos \alpha_{j}, \sqrt{2 J_{j}} \sin \alpha_{j}\right)_{j \geq 1} .
$$

For $q$ in $L^{2}\left(S^{1}\right)$, introduce $p=q-[q] \in L_{0}^{2}\left(S^{1}\right)$ and define

$$
(x(t), y(t))=\left(\sqrt{2 J_{j}} \cos \left(\omega_{j}(q) t+\alpha_{j}\right), \sqrt{2 J_{j}} \sin \left(\omega_{j}(q) t+\alpha_{j}\right)\right)_{j \geq 1}
$$

where

$$
\omega_{j}(q)=\omega_{j}(p)+12[q] \pi j .
$$

Introduce the solution operator $\mathcal{S}^{(1)}$ of $K d V, \mathcal{S}^{(1)}: L^{2}\left(S^{1}\right) \rightarrow C\left(\mathbb{R} ; L^{2}\left(S^{1}\right)\right)$ where

$$
\mathcal{S}^{(1)}(q)(t)=[q]+\Omega^{-1}(x(t), y(t)) .
$$

Recently, Bourgain [Bo1] has found weak solutions of KdV, which can be analyzed further by using Theorem 1 :

Theorem 2. Let $c \in \mathbb{R}$. Then

(i) $\mathcal{S}^{(1)}(q)$ coincides with the weak solution $\mathcal{S}_{B}(q)$ constructed by Bourgain for any initial data $q$ in $L_{c}^{2}\left(S^{1}\right)$.

(ii) Given $q_{1}, q_{2}$ in $L_{c}^{2}\left(S^{1}\right)$, there exists $M>0$ so that for any $t \in \mathbb{R}$

$$
\left\|\left(\mathcal{S}^{(1)}\left(q_{1}\right)\right)(\cdot, t)-\left(\mathcal{S}^{(1)}\left(q_{2}\right)\right)(\cdot, t)\right\|_{L^{2}\left(S^{1}\right)} \leq M(1+|t|)\left\|q_{1}-q_{2}\right\|_{L^{2}\left(S^{1}\right)} .
$$

(iii) For any $0<T<\infty \quad \mathcal{S}^{(1)}: L_{c}^{2}\left(S^{1}\right) \rightarrow C\left([-T, T] ; L_{c}^{2}\left(S^{1}\right)\right)$ is real analytic.

Remark 1. The estimate (1.9) strengthens a result of Bourgain [Bo1], Theorem 5. Instead of $M(1+|t|)$, Bourgain obtains $e^{C|t|}$ where $C>0$ is some constant depending on $q_{1}$ and $q_{2}$.

Remark 2. Recently, we have learnt that property (iii) has also been obtained by Zhang $[\mathbf{Z h}]$ using methods very different from ours.

Remark 3. McKean-Trubowitz [MT1] have constructed classical solutions of (1.1), using theta functions. However, with their approach, one has to fix an isospectral set of potentials in advance and therefore, properties of well posedness cannot be proved.

Remark 4. Note that $\mathcal{S}^{(1)}$ is not real analytic on all of $L^{2}\left(S^{1}\right)$ : for generic initial data $q$ with $\operatorname{Im}[q] \neq 0$ one sees from (1.6) and (1.7) that $K d V$ is ill posed. 
Similar results as the ones presented for $K d V$ hold for any of the equations in the $K d V$ hierarchy. As an example we consider $K d V_{2}$, i.e., the fifth order equation in the hierarchy.

$$
\partial_{t} v=\frac{1}{4} \partial_{x}^{5} v-\frac{5}{2} v \partial_{x}^{3} v-5 \partial_{x} v \partial_{x}^{2} v+\frac{15}{2} v^{2} \partial_{x} v
$$

In Section 4 we analyze the frequencies corresponding to the Hamiltonian of $K d V_{2}$ and use the results to construct weak solutions $\mathcal{S}^{(2)}(q)$ of (1.10) for initial data in $H_{c_{1}, c_{3}}^{1}\left(S^{1}\right):=\left\{q \in H^{1}\left(S^{1}\right) \mid[q]=c_{3} ; \frac{5}{8} \int_{S^{1}} q(x)^{2} d x=c_{1}\right\}$, with $c_{1}, c_{3} \in \mathbb{R}$

$$
\mathcal{S}^{(2)}: H_{c_{1}, c_{3}}^{1}\left(S^{1}\right) \rightarrow C\left(\mathbb{R}, H_{c_{1}, c_{3}}^{1}\left(S^{1}\right)\right)
$$

and prove that $\mathcal{S}^{(2)}$ has properties similar to those of $\mathcal{S}^{(1)}$.

We point out that, according to [Bo2], Bourgain's method cannot be used to obtain weak solutions of (1.10) for initial data in $H_{c}^{1}\left(S^{1}\right)$. Results similar to the ones presented in this paper hold most likely also for the defocusing nonlinear Schrödinger equation $\frac{1}{i} \partial_{t} \psi=\partial_{x}^{2} \psi-|\psi|^{2} \psi$. However we have not verified the details.

This paper is related to $[\mathbf{B K M}]$ where we present further applications of the existence of action-angle variables for $K d V$. Among other things, we prove that in a neighborhood in $H^{1}\left(S^{1}\right)$ of the elliptic fix point $q \equiv 0$, the Hamiltonian $\mathcal{H}$ admits a convergent Birkhoff normal form.

The notation in this paper is standard and coincides with the one used in $[\mathrm{Ka}]$ and $[\mathrm{BBGK}]$.

Acknowledgement. It is a pleasure to thank J. Bourgain, G. Forest, J.C. Guillot, A. Kostyuchenko, D. McLaughlin and J. Moser for helpful discussions. T. Kappeler would like to thank IHES and Université ParisNord and B. Mityagin the Center for Research in Mathematics, Guanajuato, Mexico for the kind hospitality.

\section{Auxilary results.}

In this section we prove auxilary results needed in the following section to show that the frequencies $\omega_{j}$ can be defined on the whole space $L_{0}^{2}\left(S^{1}\right)$.

Given a potential $q$ in $L_{0}^{2}\left(S^{1} ; \mathbb{C}\right)$, denote by $\left(\lambda_{j}=\lambda_{j}(q)\right)_{j \geq 0}$ the union of the periodic and antiperiodic spectrum of $-\frac{d^{2}}{d x^{2}}+q$ (with multiplicities). The eigenvalues are ordered so that $\operatorname{Re} \lambda_{0} \leq \operatorname{Re} \lambda_{1} \leq \operatorname{Re} \lambda_{3} \leq \ldots$ and, if $\operatorname{Re} \lambda_{n}=\operatorname{Re} \lambda_{n+1}$, then $\operatorname{Im} \lambda_{n} \leq \operatorname{Im} \lambda_{n+1}$.

Introduce $\tau_{j}=\left(\lambda_{2 j}+\lambda_{2 j-1}\right) / 2, \gamma_{j}=\left(\lambda_{2 j}-\lambda_{2 j-1}\right)$ and the gap interval $I_{j}=\left\{t \lambda_{2 j}+(1-t) \lambda_{2 j-1} ; 0 \leq t \leq 1\right\} \subseteq \mathbb{C}$. Note that $\tau_{j}$, but not $\gamma_{j}$ are real analytic functions on $L_{0}^{2}\left(S^{1}\right)$. Denote by $\Delta(\lambda)=\Delta(\lambda, q)$ the discriminant

$$
\Delta(\lambda):=y_{1}(1, \lambda)+\frac{d}{d x} y_{2}(1, \lambda)
$$


where $y_{1}(x, \lambda)$ and $y_{2}(x, \lambda)$ denote the fundamental solutions for $-\frac{d^{2}}{d x^{2}}+q$. Introduce $\sqrt{\Delta(\mu)^{2}-4}$ with the sign determined so that, in the case where $q$ is real valued, $\sqrt{\Delta(\mu)^{2}-4}>0$ for $\mu \in\left(-\infty, \lambda_{0}\right)$.

Theorem 2.1. Let $q_{0}$ be in $L_{0}^{2}\left(S^{1}\right)$. Then there exists a (sufficiently small) neighborhood $U_{q_{0}}$ of $q_{0}$ in $L_{0}^{2}\left(S^{1} ; \mathbb{C}\right)$ with the following properties:

For any $j \geq 1$ and $q \in U_{q_{0}}$ there exists a unique sequence

$\left(\mu_{k}^{(j)}\right)_{k \in \mathbb{N} \backslash\{j\}}$ satisfying uniformly for $j \geq 1, k \in \mathbb{N} \backslash\{j\}$

and $q$ in $U_{q_{0}}$,

$$
\left|\mu_{k}^{(j)}-\tau_{k}\right| \leq\left|\gamma_{k}\right|^{2} O\left(\frac{1}{k}\right)
$$

so that, for all $k$ in $\mathbb{N} \backslash\{j\}$,

$$
\int_{\lambda_{2 k-1}}^{\lambda_{2 k}} \frac{\varphi_{j}(\lambda) d \lambda}{\sqrt{\Delta(\lambda)^{2}-4}}=0
$$

where $\varphi_{j}(\lambda)$ is the entire function

$$
\varphi_{j}(\lambda)=\frac{1}{j^{2} \pi^{2}} \prod_{k \neq j} \frac{\mu_{k}^{(j)}-\lambda}{k^{2} \pi^{2}} .
$$

Moreover, the $\mu_{k}^{(j)}$ 's are analytic functions on $U_{q_{0}}$. In case where $q$ is real valued, the $\mu_{k}^{(j)}$ are real valued and satisfy

$$
\lambda_{2 k-1} \leq \mu_{k}^{(j)} \leq \lambda_{2 k} \quad(\forall j \neq \ell) .
$$

Rephrasing the above results, Theorem 2.1 states that there exist 1-forms $\Omega_{j}(\lambda)=\frac{\varphi_{j}(\lambda) d \lambda}{\sqrt{\Delta(\lambda)^{2}-4}}$ in the Hilbert space of quadratically integrable holomorphic 1-forms on the (open) Riemann surface $y=\sqrt{\Delta(\lambda)^{2}-4}$ which satisfy (2.2) and that the zeroes of these 1-forms $\Omega_{j}(\lambda)$ depend analytically on $q$. Actually, the $\Omega_{j}^{\prime}$ s are a basis in the space of these 1 -forms. If $q \in U_{q_{0}}$ is real valued, the existence of such a basis of 1 -forms $\Omega_{j}(\lambda)$ with a product representation (2.3) has been proved by [MT2] (cf also [FKT]). A proof of Theorem 2.1 is provided in the Appendix.

The rest of this section is devoted to a refinement of the asymptotics (2.1). First note that, in the case where $\gamma_{k}=0, \mu_{k}^{(j)}=\tau_{k}$. In case $\gamma_{k} \neq 0$, we make a change of coordinates in $(2.2), \lambda(t)=\tau_{k}+\frac{\gamma_{k}}{2} t$, which leads to

$$
\int_{-1}^{1} \frac{\varphi_{j}(\lambda(t)) \frac{\gamma_{k}}{2} d t}{\sqrt{\Delta(\lambda(t))^{2}-4}}=0 .
$$


It is convenient to introduce

$$
\begin{aligned}
& \Delta_{\alpha}(t)=\left(\lambda_{2 \alpha}-\lambda(t)\right)\left(\lambda_{2 \alpha-1}-\lambda(t)\right) \quad(\alpha \geq 1) \\
& \Delta_{0}(t)=\left(\lambda(t)-\lambda_{0}\right) .
\end{aligned}
$$

Notice that

$$
\Delta_{\alpha}(t)=\left(\tau_{\alpha}+\frac{\gamma_{\alpha}}{2}-\lambda(t)\right)\left(\tau_{\alpha}-\frac{\gamma_{\alpha}}{2}-\lambda(t)\right)=\left(\tau_{\alpha}-\lambda(t)\right)^{2}-\left(\frac{\gamma_{\alpha}}{2}\right)^{2} .
$$

Therefore, with $\tau_{k}-\lambda(t)=\tau_{k}-\tau_{k}-\frac{\gamma_{k}}{2} t$,

$$
-\Delta_{k}(t)=\left(\lambda_{2 k}-\lambda(t)\right)\left(\lambda(t)-\lambda_{2 k-1}\right)=\left(\frac{\gamma_{k}}{2}\right)^{2}\left(1-t^{2}\right)
$$

and

$$
\begin{aligned}
& \Delta(\lambda(t))^{2}-4=4\left(\lambda_{0}-\lambda(t)\right) \prod_{\alpha \geq 1} \frac{\left(\lambda_{2 \alpha}-\lambda(t)\right)\left(\lambda_{2 \alpha-1}-\lambda(t)\right)}{\left(\alpha^{2} \pi^{2}\right)^{2}} \\
&= 4 \Delta_{0}(t)\left(\frac{\gamma_{k}}{2}\right)^{2} \frac{\left(1-t^{2}\right)}{k^{4} \pi^{4}} \prod_{\alpha \neq k} \frac{\Delta_{\alpha}(t)}{\left(\alpha^{2} \pi^{2}\right)^{2}} \\
&= 4 \Delta_{0}(t)\left(\frac{\gamma_{k}}{2}\right)^{2} \frac{1-t^{2}}{k^{4} \pi^{4}} \frac{\Delta_{j}(t)}{\left(j^{2} \pi^{2}\right)^{2}} \prod_{\alpha \in \mathbb{N} \backslash\{j, k\}} \frac{\left(\tau_{\alpha}-\lambda(t)\right)^{2}}{\left(\alpha^{2} \pi^{2}\right)^{2}} \\
& \cdot \prod_{\alpha \in \mathbb{N} \backslash\{j, k\}}\left(1-\frac{\left(\frac{\gamma_{\alpha}}{2}\right)^{2}}{\left(\tau_{\alpha}-\lambda(t)\right)^{2}}\right) .
\end{aligned}
$$

Further we introduce

$$
\xi_{\alpha}^{(j)}:=\mu_{\alpha}^{(j)}-\tau_{\alpha}
$$

This leads to

$$
\begin{aligned}
\varphi_{j}(\lambda(t)) & =\frac{1}{j^{2} \pi^{2}} \prod_{\alpha \neq j} \frac{\tau_{\alpha}-\lambda(t)+\xi_{\alpha}^{(j)}}{\alpha^{2} \pi^{2}} \\
& =\frac{1}{j^{2} \pi^{2}} \frac{\xi_{k}^{(j)}-\frac{\gamma_{k}}{2} t}{k^{2} \pi^{2}} \prod_{\alpha \in \mathbb{N} \backslash\{j, k\}} \frac{\tau_{\alpha}-\lambda(t)}{\alpha^{2} \pi^{2}}\left(1+\frac{\xi_{\alpha}^{(j)}}{\tau_{\alpha}-\lambda(t)}\right) .
\end{aligned}
$$

Combining (2.6) and (2.8) we obtain

$$
\frac{\varphi_{j}(\lambda(t))}{\sqrt{\Delta(\lambda(t))^{2}-4}}=\frac{\xi_{k}^{(j)}-\frac{\gamma_{k}}{2} t}{2 \frac{\gamma_{k}}{2} \sqrt{\Delta_{0}(t)} \sqrt{\Delta_{j}(t)}} \frac{1}{\sqrt{1-t^{2}}} \prod_{\alpha \in \mathbb{N} \backslash\{j, k\}}\left(1+\frac{\xi_{\alpha}^{(j)}}{\tau_{\alpha}-\lambda(t)}\right)
$$




$$
\prod_{\alpha \in \mathbb{N} \backslash\{j, k\}}\left(1-\frac{\left(\frac{\gamma_{\alpha}}{2}\right)^{2}}{\left(\tau_{\alpha}-\lambda(t)\right)^{2}}\right)^{-1 / 2} .
$$

The Equation (2.4) then takes the form

$$
\begin{gathered}
\xi_{k}^{(j)} \int_{-1}^{1} \frac{d t}{\sqrt{\Delta_{0}(t)} \sqrt{\Delta_{j}(t)} \sqrt{1-t^{2}}} \\
\cdot \prod_{\alpha \in \mathbb{N} \backslash\{j, k\}}\left(1+\frac{\xi_{\alpha}^{(j)}}{\tau_{\alpha}-\lambda(t)}\right)\left(1-\frac{\left(\frac{\gamma_{\alpha}}{2}\right)^{2}}{\left(\tau_{\alpha}-\lambda(t)\right)^{2}}\right)^{-1 / 2} \\
=\frac{\gamma_{k}}{2} \int_{-1}^{1} \frac{t d t}{\sqrt{\Delta_{0}(t)} \sqrt{\Delta_{j}(t)} \sqrt{1-t^{2}}} \\
\cdot \prod_{\alpha \in \mathbb{N} \backslash\{j, k\}}\left(1+\frac{\xi_{\alpha}^{(j)}}{\tau_{\alpha}-\lambda(t)}\right)\left(1-\frac{\left(\frac{\gamma_{\alpha}}{2}\right)^{2}}{\left(\tau_{\alpha}-\lambda(t)\right)^{2}}\right)^{-1 / 2} .
\end{gathered}
$$

Now introduce

$$
A_{j k}(t) \equiv A_{j k}(t, q):=\prod_{\alpha \in \mathbb{N} \backslash\{j, k\}}\left(1+\frac{\xi_{\alpha}^{(j)}}{\tau_{\alpha}-\lambda(t)}\right)
$$

and

$$
B_{j k}(t) \equiv B_{j k}(t, q):=\prod_{\alpha \in \mathbb{N} \backslash\{j, k\}}\left(1-\frac{\left(\frac{\gamma_{\alpha}}{2}\right)^{2}}{\left(\tau_{\alpha}-\lambda(t)\right)^{2}}\right)^{-1 / 2} .
$$

After multiplying left and right hand side of (2.10) with the $t$-independent nonvanishing quantity

$$
\frac{\sqrt{\Delta_{0}(0)} \sqrt{\Delta_{j}(0)}}{A_{j k}(0) B_{j k}(0)}
$$

the Equation (2.10) reads

$$
\xi_{k}^{(j)}=\frac{\gamma_{k}}{2} \frac{\int_{-1}^{1} \frac{t d t}{\sqrt{1-t^{2}}} \sqrt{\frac{\Delta_{0}(0)}{\Delta_{0}(t)}} \sqrt{\frac{\Delta_{j}(0)}{\Delta_{j}(t)}} \frac{A_{j k}(t)}{A_{j k}(0)} \frac{B_{j k}(t)}{B_{j k}(0)}}{\int_{-1}^{1} \frac{d t}{\sqrt{1-t^{2}}} \sqrt{\frac{\Delta_{0}(0)}{\Delta_{0}(t)}} \sqrt{\frac{\Delta_{j}(0)}{\Delta_{j}(t)}} \frac{A_{j k}(t)}{A_{j k}(0)} \frac{B_{j k}(t)}{B_{j k}(0)}} .
$$

It will turn out that for $k \geq N$, with $N$ sufficiently large, the denominator in (2.11) is different from 0 . Thus the quotient in (2.11) is well defined at least for k sufficiently large. To simplify (2.11), we write

$$
C_{j k}(t) \equiv C_{j k}(t, q):=\sqrt{\frac{\Delta_{0}(0)}{\Delta_{0}(t)}} \sqrt{\frac{\Delta_{j}(0)}{\Delta_{j}(t)}} \frac{A_{j k}(t)}{A_{j k}(0)} \frac{B_{j k}(t)}{B_{j k}(0)}
$$


and obtain

$$
\xi_{k}^{(j)}=\frac{\gamma_{k}}{2} \frac{\int_{0}^{1} \frac{t d t}{\sqrt{1-t^{2}}}\left(C_{j k}(t)-C_{j k}(-t)\right)}{\int_{0}^{1} \frac{d t}{\sqrt{1-t^{2}}}\left(C_{j k}(t)+C_{j k}(-t)\right)} .
$$

To obtain asymptotics for $\xi_{k}^{(j)}$ we estimate

$$
\sqrt{\frac{\Delta_{j}(0)}{\Delta_{j}( \pm t)}}-1, \sqrt{\frac{\Delta_{0}(0)}{\Delta_{0}( \pm t)}}-1, \frac{A_{j k}( \pm t)}{A_{j k}(0)}-1 \text { and } \frac{B_{j k}( \pm t)}{B_{j k}(0)}-1 .
$$

Recall that for $q \in L_{0}^{2}\left(S^{1}\right), \sum_{k}\left|\gamma_{k}\right|^{2}<\infty$.

Lemma 2.2. Let $q_{0} \in L_{0}^{2}\left(S^{1}\right)$. Then there exist a neighborhood $U_{q_{0}}$ of $q_{0}$ in $L_{0}^{2}\left(S^{1} ; \mathbb{C}\right)$ and $C>0$, independent of $j$ and $k$, so that, for $q \in U_{q_{0}}$ and $j \neq k$,

$$
\sup _{0 \leq t \leq 1}\left|\frac{A_{j k}( \pm t)}{A_{j k}(0)}-1\right| \leq C \frac{\left|\gamma_{k}\right|}{k^{3}}\left(\sum_{|\alpha-k| \leq \frac{k}{2}}\left|\gamma_{\alpha}\right|^{2}+\frac{1}{k}\right)
$$

Proof. Notice that

$$
\sup _{0 \leq t \leq 1}\left|\frac{A_{j k}( \pm t)}{A_{j k}(0)}-1\right| \leq \sup _{|t| \leq 1}\left|\frac{\frac{\partial}{\partial t} A_{j k}(t)}{A_{j k}(0)}\right| .
$$

Recall that $A_{j k}(t)=\prod_{\alpha \in \mathbb{N} \backslash\{j, k\}}\left(1+\frac{\xi_{\alpha}^{(j)}}{\tau_{\alpha}-\lambda(t)}\right)$ and $\lambda(t)=\tau_{k}+\frac{\gamma_{k}}{2} t$ and therefore,

$$
\frac{1}{A_{j k}(0)} \frac{\partial}{\partial t} A_{j k}(t)=\frac{A_{j k}(t)}{A_{j k}(0)}\left(\sum_{\alpha \neq j, k} \frac{1}{\left(1+\frac{\xi_{\alpha}^{(j)}}{\tau_{\alpha}-\lambda(t)}\right)} \frac{(-1) \xi_{\alpha}^{(j)}}{\left(\tau_{\alpha}-\lambda(t)\right)^{2}}\left(-\frac{\gamma_{k}}{2}\right)\right)
$$

This leads to,

$$
\begin{aligned}
\left|\frac{1}{A_{j k}(0)} \frac{\partial}{\partial t} A_{j k}(t)\right| \leq & \widehat{C}\left|\gamma_{k}\right|\left(\sum_{|\alpha-k| \geq \frac{k}{2}} \frac{\left|\xi_{\alpha}^{(j)}\right|}{\left|\tau_{\alpha}-\lambda(t)\right|^{2}}\right) \\
& +\widehat{C}\left|\gamma_{k}\right|\left(\sum_{|\alpha-k| \leq \frac{k}{2}} \frac{\left|\xi_{\alpha}^{(j)}\right|}{\left|\tau_{\alpha}-\lambda(t)\right|^{2}}\right) .
\end{aligned}
$$

Notice that, for any $\alpha$ with $|\alpha-k| \geq \frac{k}{2}$,

$$
\frac{1}{\left|\tau_{\alpha}-\lambda(t)\right|^{2}} \leq \frac{C}{\left(\alpha^{2}-k^{2}\right)^{2}}=O\left(\frac{1}{k^{4}}\right)
$$


Therefore,

$$
\sum_{|\alpha-k| \geq \frac{k}{2}} \frac{\left|\xi_{\alpha}^{(j)}\right|}{\left|\tau_{\alpha}-\lambda(t)\right|^{2}} \leq \frac{C}{k^{4}} \sum_{\alpha}\left|\xi_{\alpha}^{(j)}\right|=O\left(\frac{1}{k^{4}}\right) .
$$

For $\alpha$ with $|\alpha-k| \leq \frac{k}{2}$ we use the estimate $\xi_{\alpha}^{(j)}=\gamma_{\alpha}^{2} O\left(\frac{1}{k}\right)$ to conclude that

$$
\sum_{|\alpha-k| \leq \frac{k}{2}} \frac{\left|\xi_{\alpha}^{(j)}\right|}{\left|\tau_{\alpha}-\lambda(t)\right|^{2}} \leq \frac{C}{k^{3}} \sum_{|\alpha-k| \leq \frac{k}{2}}\left|\gamma_{\alpha}\right|^{2}
$$

Combining (2.14) - (2.16) yields (2.13).

Lemma 2.3. Let $q_{0} \in L_{0}^{2}\left(S^{1}\right)$. Then there exist a neighborhood $U_{q_{0}}$ of $q_{0}$ in $L_{0}^{2}\left(S^{1} ; \mathbb{C}\right)$ and $C>0$, independent of $j$ and $k$, so that for $q \in U_{q_{0}}$ and $j \neq k$,

$$
\sup _{0 \leq t \leq 1}\left|\frac{B_{j k}( \pm t)}{B_{j k}(0)}-1\right| \leq \frac{C\left|\gamma_{k}\right|}{k^{3}}\left(\sum_{|\alpha-k| \leq \frac{k}{2}}\left|\gamma_{\alpha}\right|^{2}+\frac{1}{k^{3}}\right)
$$

Proof. Notice that

$$
\sup _{0 \leq t \leq 1}\left|\frac{B_{j k}( \pm t)}{B_{j k}(0)}-1\right| \leq \sup _{|t| \leq 1}\left|\frac{\frac{\partial}{\partial t} B_{j k}(t)}{B_{j k}(0)}\right| .
$$

Recall that $B_{j k}(t)=\prod_{\alpha \neq j, k}\left(1-\frac{\left(\frac{\gamma_{\alpha}}{2}\right)^{2}}{\left(\tau_{\alpha}-\lambda(t)\right)^{2}}\right)^{-1 / 2}$ to conclude that

$$
\frac{\partial}{\partial t} B_{j k}(t)=B_{j k}(t)\left\{\sum_{\alpha \in \mathbb{N} \backslash\{j, k\}} \frac{-1 / 2}{\left(1-\frac{\left(\frac{\gamma_{\alpha}}{2}\right)^{2}}{\left(\tau_{\alpha}-\lambda(t)\right)^{2}}\right)} \cdot \frac{2\left(\frac{\gamma_{\alpha}}{2}\right)^{2}}{\left(\tau_{\alpha}-\lambda(t)\right)^{3}}\left(\frac{-\gamma_{k}}{2}\right)\right\} .
$$

This leads to

$$
\begin{aligned}
\frac{1}{\left|B_{j k}(0)\right|}\left|\frac{\partial}{\partial t} B_{j k}(t)\right| & \leq \frac{\left|B_{j k}(t)\right|}{\left|B_{j k}(0)\right|}\left\{\sum_{\alpha \in \mathbb{N} \backslash\{j, k\}} C\left|\gamma_{k}\right| \frac{1}{\left|\tau_{\alpha}-\lambda(t)\right|^{3}}\left|\gamma_{\alpha}\right|^{2}\right\} \\
& \leq C\left|\gamma_{k}\right|\left(\sum_{|\alpha-k| \geq \frac{k}{2}} \frac{\left|\gamma_{\alpha}\right|^{2}}{\left|\tau_{\alpha}-\lambda(t)\right|^{3}}+\sum_{|\alpha-k| \leq \frac{k}{2}} \frac{\left|\gamma_{\alpha}\right|^{2}}{\left|\tau_{\alpha}-\lambda(t)\right|^{3}}\right)
\end{aligned}
$$




$$
\leq C\left|\gamma_{k}\right|\left(\frac{C}{k^{6}}+\frac{1}{k^{3}} \sum_{|\alpha-k| \leq \frac{k}{2}}\left|\gamma_{\alpha}\right|^{2}\right)
$$

Lemma 2.4. Let $q_{0} \in L_{0}^{2}\left(S^{1}\right)$. Then there exist a neighborhood $U_{q_{0}}$ in $L_{0}^{2}\left(S^{1} ; \mathbb{C}\right)$ and $C>0$, independent of $k$, such that for any $q$ in $U_{q_{0}}$

$$
\sup _{|t| \leq 1}\left|\left(\frac{\tau_{k}-\lambda_{0}}{\lambda(t)-\lambda_{0}}\right)^{1 / 2}-\left(1-\frac{\gamma_{k}}{4} \frac{1}{\tau_{k}-\lambda_{0}} t\right)\right| \leq C \frac{\left|\gamma_{k}\right|^{2}}{k^{4}}
$$

and

$$
\sup _{0 \leq t \leq 1}\left|\left(\frac{\tau_{k}-\lambda_{0}}{\lambda( \pm t)-\lambda_{0}}\right)^{1 / 2}-1\right| \leq C \frac{\left|\gamma_{k}\right|}{k^{2}}
$$

Proof. Notice that

$$
\begin{gathered}
\frac{d}{d t}\left(\frac{\tau_{k}-\lambda_{0}}{\lambda(t)-\lambda_{0}}\right)^{1 / 2}=\left(\frac{\tau_{k}-\lambda_{0}}{\lambda(t)-\lambda_{0}}\right)^{1 / 2} \frac{(-1 / 2)}{\lambda(t)-\lambda_{0}} \frac{\gamma_{k}}{2}, \\
\frac{d^{2}}{d t^{2}}\left(\frac{\tau_{k}-\lambda_{0}}{\lambda(t)-\lambda_{0}}\right)^{1 / 2}=\left(\frac{\tau_{k}-\lambda_{0}}{\lambda(t)-\lambda_{0}}\right)^{1 / 2} \frac{3 / 4}{\left(\lambda(t)-\lambda_{0}\right)^{2}}\left(\frac{\gamma_{k}}{2}\right)^{2}, \\
\sup _{0 \leq t \leq 1}\left|\left(\frac{\tau_{k}-\lambda_{0}}{\lambda( \pm t)-\lambda_{0}}\right)^{1 / 2}-1\right| \leq \sup _{|t| \leq 1}\left|\frac{d}{d t}\left(\frac{\tau_{k}-\lambda_{0}}{\lambda(t)-\lambda_{0}}\right)^{1 / 2}\right| \leq C \frac{1}{k^{2}}\left|\gamma_{k}\right| .
\end{gathered}
$$

This proves (2.19). Taylor's formula and (2.20) lead to (2.18).

The difference $\sqrt{\frac{\Delta_{j}(0)}{\Delta_{j}( \pm t)}}-1$ needs to be treated with more care as

$$
\sup _{|t| \leq 1}\left|\sqrt{\frac{\Delta_{j}(0)}{\Delta_{j}( \pm t)}}-1\right| \leq \frac{C\left|\gamma_{k}\right|}{\left|\tau_{k}-\tau_{j}\right|}=\left|\gamma_{k}\right| O\left(\frac{1}{k}\right)
$$

Lemma 2.5. Let $q_{0} \in L_{0}^{2}\left(S^{1}\right)$. Then there exist a neighborhood $U_{q_{0}}$ of $q_{0}$ in $L_{0}^{2}\left(S^{1} ; \mathbb{C}\right)$ and $C>0$, independent of $j$ and $k$, such that for any $q$ in $U_{q_{0}}$ and $j \neq k$,

$$
\left|\sqrt{\frac{\Delta_{j}(0)}{\Delta_{j}(t)}}-\left(1+\frac{1}{2} \frac{\gamma_{k}\left(\tau_{j}-\tau_{k}\right)}{\Delta_{j}(0)} t+\left(\frac{-1}{8} \frac{\gamma_{k}^{2}}{\Delta_{j}(0)}+\frac{3}{8} \frac{\gamma_{k}^{2}\left(\tau_{j}-\tau_{k}\right)^{2}}{\Delta_{j}(0)^{2}}\right) t^{2}\right)\right|
$$


$\leq C \frac{\left|\gamma_{k}\right|^{3}}{\left(\tau_{k}-\tau_{j}\right)^{3}}$

Proof. Recall that

$\Delta_{j}(t)=\left(\tau_{j}-\lambda(t)\right)^{2}-\left(\frac{\gamma_{j}}{2}\right)^{2}=\left(\tau_{j}-\tau_{k}\right)^{2}-\left(\frac{\gamma_{k}}{2}\right)^{2}-2 \frac{\gamma_{k}}{2}\left(\tau_{j}-\tau_{k}\right) t+\left(\frac{\gamma_{k}}{2}\right)^{2} t^{2}$

and therefore

$$
\begin{aligned}
& \left.\left.\left(\frac{\Delta_{j}(0)}{\Delta_{j}(t)}\right)=\left(\frac{1}{1+\frac{\gamma_{k}\left(\tau_{k}-\tau_{j}\right) t+\left(\frac{\gamma_{k}}{2}\right)^{2} t^{2}}{\Delta_{j}(0)}}\right)^{1 / 2}\right)^{3 / 2}\left(-\frac{1}{2}\right) \frac{\gamma_{k}\left(\tau_{k}-\tau_{j}\right)+2\left(\frac{\gamma_{k}}{2}\right)^{2} t}{\Delta_{j}(0)}\right\}\left.\right|_{t=0} t \\
& =1+\left\{\left(\frac{1}{1+\frac{\gamma_{k}\left(\tau_{k}-\tau_{j}\right) t+\left(\frac{\gamma_{k}}{2}\right)^{2} t^{2}}{\Delta_{j}(0)}}\right)^{5 / 2}\right)^{2}\left(-\frac{1}{2}\right)\left(-\frac{3}{2}\right)\left(\frac{\gamma_{k}\left(\tau_{k}-\tau_{j}\right)+2\left(\frac{\gamma_{k}}{2}\right)^{2} t}{\Delta_{j}(0)}\right)^{2} \\
& \left.\quad+\left\{\left(\frac{1}{1+\frac{\gamma_{k}\left(\tau_{k}-\tau_{j}\right) t+\left(\frac{\gamma_{k}}{2}\right)^{2} t^{2}}{\Delta_{j}(0)}}\right)^{3 / 2}\right)^{1}\left(-\frac{1}{2}\right) \frac{2\left(\frac{\gamma_{k}}{2}\right)^{2}}{\Delta_{j}(0)}\right\}\left.\right|_{t=0} ^{\frac{t^{2}}{2}+\frac{\gamma_{k}^{3}}{\left(\tau_{k}-\tau_{j}\right)^{3}} O(1)} \\
& \quad+\left(\frac{\gamma_{k}\left(\tau_{k}-\tau_{j}\right) t+\left(\frac{\gamma_{k}}{2}\right)^{2} t^{2}}{\Delta_{j}(0)}\right)^{2}
\end{aligned}
$$

which leads to (2.21).

Lemma 2.6. Let $q_{0} \in L_{0}^{2}\left(S^{1}\right)$. Then there exist a neighborhood $U_{q_{0}}$ of $q_{0}$ in $L_{0}^{2}\left(S^{1} ; \mathbb{C}\right)$ and $C>0$, independent of $j$ and $k$, such that for any $q$ in $U_{q_{0}}$, and $j \neq k$,

$$
\begin{aligned}
& \left|\frac{\gamma_{k}}{2} \frac{\int_{0}^{1} \frac{t d t}{\sqrt{1-t^{2}}}\left(\sqrt{\frac{\Delta_{j}(0)}{\Delta_{j}(t)}}-\sqrt{\frac{\Delta_{j}(0)}{\Delta_{j}(-t)}}\right)}{\int_{0}^{1} \frac{d t}{\sqrt{1-t^{2}}}\left(\sqrt{\frac{\Delta_{j}(0)}{\Delta_{j}(t)}}+\sqrt{\frac{\Delta_{j}(0)}{\Delta_{j}(-t)}}\right)}-\frac{1}{8} \frac{\left(-\gamma_{k}^{2}\right)}{\tau_{k}-\tau_{j}}\right| \\
& \leq \frac{C}{\left(\tau_{k}-\tau_{j}\right)^{3}}\left|\gamma_{k}\right|^{2}\left(\left|\gamma_{j}\right|^{2}+\left|\gamma_{k}\right|\right) .
\end{aligned}
$$

Proof. Using Lemma 2.5, we obtain

$$
\begin{aligned}
& \int_{0}^{1} \frac{t d t}{\sqrt{1-t^{2}}}\left(\sqrt{\frac{\Delta_{j}(0)}{\Delta_{j}(t)}}-\sqrt{\frac{\Delta_{j}(0)}{\Delta_{j}(-t)}}\right) \\
& =\frac{\gamma_{k}\left(\tau_{j}-\tau_{k}\right)}{\Delta_{j}(0)} \int_{0}^{1} \frac{t^{2} d t}{\sqrt{1-t^{2}}}+\frac{\gamma_{k}^{3}}{\left(\tau_{k}-\tau_{j}\right)^{3}} O(1)
\end{aligned}
$$


and

$$
\begin{aligned}
& \int_{0}^{1} \frac{d t}{\sqrt{1-t^{2}}}\left(\sqrt{\frac{\Delta_{j}(0)}{\Delta_{j}(t)}}+\sqrt{\frac{\Delta_{j}(0)}{\Delta_{j}(-t)}}\right) \\
& =2 \int_{0}^{1} \frac{d t}{\sqrt{1-t^{2}}}+2\left(\frac{-1}{8} \frac{\gamma_{k}^{2}}{\Delta_{j}(0)}+\frac{3}{8} \frac{\gamma_{k}^{2}\left(\tau_{j}-\tau_{k}\right)^{2}}{\Delta_{j}(0)^{2}}\right) \int_{0}^{1} \frac{t^{2} d t}{\sqrt{1-t^{2}}} \\
& +\frac{\gamma_{k}^{3}}{\left(\tau_{k}-\tau_{j}\right)^{3}} O(1) .
\end{aligned}
$$

Notice that

$$
\int_{0}^{1} \frac{d t}{\sqrt{1-t^{2}}}=\frac{\pi}{2} ; \int_{0}^{1} \frac{t^{2} d t}{\sqrt{1-t^{2}}}=\frac{\pi}{4}
$$

Therefore

$$
\begin{aligned}
& \frac{\gamma_{k}}{2} \frac{\int_{0}^{1} \frac{t d t}{\sqrt{1-t^{2}}}\left(\sqrt{\frac{\Delta_{j}(0)}{\Delta_{j}(t)}}-\sqrt{\frac{\Delta_{j}(0)}{\Delta_{j}(-t)}}\right)}{\int_{0}^{1} \frac{d t}{\sqrt{1-t^{2}}}\left(\sqrt{\frac{\Delta_{j}(0)}{\Delta_{j}(t)}}+\sqrt{\frac{\Delta_{j}(0)}{\Delta_{j}(-t)}}\right)} \\
& =\frac{\gamma_{k}}{2} \frac{\frac{\gamma_{k}\left(\tau_{j}-\tau_{k}\right)}{\Delta_{j}(0)} \frac{\pi}{4}\left(1+\frac{\gamma_{k}^{2}}{\left(\tau_{k}-\tau_{j}\right)^{3}} O(1)\right)}{\pi\left(1-\left(\frac{1}{16} \frac{\gamma_{k}^{2}}{\Delta_{j}(0)}-\frac{3}{16} \frac{\gamma_{k}^{2}\left(\tau_{j}-\tau_{k}\right)^{2}}{\Delta_{j}(0)^{2}}\right)+\frac{\gamma_{k}^{3}}{\left(\tau_{k}-\tau_{j}\right)^{3}} O(1)\right)} \\
& =\frac{\gamma_{k}^{2}}{8} \frac{\tau_{j}-\tau_{k}}{\Delta_{j}(0)}+\frac{\gamma_{k}^{3}}{\left(\tau_{k}-\tau_{j}\right)^{3}} O(1) .
\end{aligned}
$$

Recall that $\Delta_{j}(0)=\left(\tau_{j}-\tau_{k}\right)^{2}-\left(\frac{\gamma_{j}}{2}\right)^{2}$. Therefore

$$
\frac{\tau_{j}-\tau_{k}}{\Delta_{j}(0)}=\frac{1}{\left(\tau_{j}-\tau_{k}\right)} \frac{1}{1-\frac{\left(\gamma_{j} / 2\right)^{2}}{\left(\tau_{j}-\tau_{k}\right)^{2}}}=\frac{1}{\tau_{j}-\tau_{k}}+\frac{\left(\gamma_{j} / 2\right)^{2}}{\left(\tau_{j}-\tau_{k}\right)^{3}} O(1) .
$$

Combining (2.25) and (2.26) we obtain

$$
\frac{\gamma_{k}}{2} \frac{\int_{0}^{1} \frac{t d t}{\sqrt{1-t^{2}}}\left(\sqrt{\frac{\Delta_{j}(0)}{\Delta_{j}(t)}}-\sqrt{\frac{\Delta_{j}(0)}{\Delta_{j}(-t)}}\right)}{\int_{0}^{1} \frac{d t}{\sqrt{1-t^{2}}}\left(\sqrt{\frac{\Delta_{j}(0)}{\Delta_{j}(t)}}+\sqrt{\frac{\Delta_{j}(0)}{\Delta_{j}(-t)}}\right)}=-\frac{\gamma_{k}^{2}}{8} \frac{1}{\tau_{k}-\tau_{j}}+\gamma_{k}^{2} \frac{\left(\gamma_{k}+\gamma_{j}^{2}\right)}{\left(\tau_{k}-\tau_{j}\right)^{3}} O(1) .
$$

Theorem 2.7. Let $q_{0} \in L_{0}^{2}\left(S^{1}\right)$. Then there exists a neighborhood $U_{q_{0}}$ of $q_{0}$ in $L_{0}^{2}\left(S^{1} ; \mathbb{C}\right), k_{0} \geq 1$ and $C>0$ such that $\forall q \in U_{q_{0}}, \forall j$ and $k \geq k_{0}$ with $k \neq j$,

$$
\left|\xi_{k}^{(j)}-\left(-\frac{1}{2} \frac{\left(\gamma_{k} / 2\right)^{2}}{\tau_{k}-\tau_{j}}-\frac{\gamma_{k}^{2}}{16} \frac{1}{\tau_{k}-\lambda_{0}}\right)\right|
$$




$$
\leq \frac{C\left|\gamma_{k}\right|^{2}}{k^{3}}\left(\left|\gamma_{k}\right|+\left|\gamma_{j}\right|^{2}+\sum_{|\alpha-k| \leq \frac{k}{2}}\left|\gamma_{\alpha}\right|^{2}+O\left(\frac{1}{k}\right)\right)
$$

Proof. Recall that

$$
\begin{aligned}
& \xi_{k}^{(j)}\left(\int_{0}^{1} \frac{d t}{\sqrt{1-t^{2}}}\left(C_{j k}(t)+C_{j k}(-t)\right)\right) \\
& =\frac{\gamma_{k}}{2} \int_{0}^{1} \frac{t d t}{\sqrt{1-t^{2}}}\left(C_{j k}(t)-C_{j k}(-t)\right) .
\end{aligned}
$$

Write

$$
\begin{aligned}
C_{j k}(t)= & \sqrt{\frac{\Delta_{j}(0)}{\Delta_{j}(t)}} \\
& +\sqrt{\frac{\Delta_{j}(0)}{\Delta_{j}(t)}}\left(\sqrt{\frac{\Delta_{0}(0)}{\Delta_{0}(t)}}-1\right) \\
& +\sqrt{\frac{\Delta_{j}(0)}{\Delta_{j}(t)}} \sqrt{\frac{\Delta_{0}(0)}{\Delta_{0}(t)}}\left(\frac{A_{j k}(t)}{A_{j k}(0)}-1\right) \\
& +\sqrt{\frac{\Delta_{j}(0)}{\Delta_{j}(t)}} \sqrt{\frac{\Delta_{0}(0)}{\Delta_{0}(t)}} \frac{A_{j k}(t)}{A_{j k}(0)}\left(\frac{B_{j k}(t)}{B_{j k}(0)}-1\right) .
\end{aligned}
$$

Notice that, by Lemma 2.5

$$
\begin{aligned}
\sqrt{\frac{\Delta_{j}(0)}{\Delta_{j}(t)}}\left(\sqrt{\frac{\Delta_{0}(0)}{\Delta_{0}(t)}}-1\right) \\
=\left(1+\frac{1}{2} \frac{\gamma_{k}\left(\tau_{j}-\tau_{k}\right)}{\Delta_{j}(0)} t+\left(-\frac{1}{8} \frac{\gamma_{k}^{2}}{\Delta_{j}(0)}+\frac{3}{8} \frac{\gamma_{k}^{2}\left(\tau_{j}-\tau_{k}\right)^{2}}{\Delta_{j}(0)^{2}}\right) t^{2}+O\left(\frac{\left|\gamma_{k}\right|^{3}}{k^{3}}\right)\right) \\
\quad \cdot\left(-\frac{\gamma_{k}}{4} \frac{1}{\tau_{k}-\lambda_{0}} t+O\left(\frac{\left|\gamma_{k}\right|^{2}}{k^{4}}\right)\right) \\
=-\frac{\gamma_{k}}{4} \frac{1}{\tau_{k}-\lambda_{0}} t+\frac{1}{2} \frac{\gamma_{k}\left(\tau_{j}-\tau_{k}\right)}{\Delta_{j}(0)}\left(-\frac{\gamma_{k}}{4}\right) \frac{1}{\tau_{k}-\lambda_{0}} t^{2}+O\left(\frac{\left|\gamma_{k}\right|^{2}}{k^{4}}\right) .
\end{aligned}
$$

Therefore

$$
\begin{gathered}
\frac{\gamma_{k}}{2} \int_{0}^{1} \frac{t d t}{\sqrt{1-t^{2}}}\left\{\left(\sqrt{\frac{\Delta_{j}(0)}{\Delta_{j}(t)}}\left(\sqrt{\frac{\Delta_{0}(0)}{\Delta_{0}(t)}}-1\right)\right.\right. \\
\left.\left.-\sqrt{\frac{\Delta_{j}(0)}{\Delta_{j}(-t)}}\left(\sqrt{\frac{\Delta_{0}(0)}{\Delta_{0}(-t)}}-1\right)\right)\right\}
\end{gathered}
$$




$$
\begin{aligned}
& =\frac{\gamma_{k}}{2} \int_{0}^{1} \frac{t^{2} d t}{\sqrt{1-t^{2}}} 2\left(-\frac{\gamma_{k}}{4} \frac{1}{\tau_{k}-\lambda_{0}}\right)+0+O\left(\frac{\left|\gamma_{k}\right|^{3}}{k^{4}}\right) \\
& =-\frac{\gamma_{k}^{2}}{16} \frac{\pi}{\tau_{k}-\lambda_{0}}+O\left(\frac{\gamma_{k}^{3}}{k^{4}}\right)
\end{aligned}
$$

and

$$
\begin{aligned}
& \int_{0}^{1} \frac{d t}{\sqrt{1-t^{2}}}\left\{\sqrt{\frac{\Delta_{j}(0)}{\Delta_{j}(t)}}\left(\sqrt{\frac{\Delta_{0}(0)}{\Delta_{0}(t)}}-1\right)+\sqrt{\frac{\Delta_{j}(0)}{\Delta_{j}(-t)}}\left(\sqrt{\frac{\Delta_{j}(0)}{\Delta_{j}(-t)}}-1\right)\right\} \\
& =+\frac{\gamma_{k}^{2}}{4} \frac{\left(\tau_{k}-\tau_{j}\right)}{\Delta_{j}(0)} \frac{1}{\left(\tau_{k}-\lambda_{0}\right)} \int_{0}^{1} \frac{t^{2} d t}{\sqrt{1-t^{2}}}+O\left(\frac{\left|\gamma_{k}\right|^{2}}{k^{4}}\right) .
\end{aligned}
$$

By Lemma 2.2, we conclude

$$
\begin{aligned}
& \mid \frac{\gamma_{k}}{2} \int_{0}^{1} \frac{t d t}{\sqrt{1-t^{2}}}\left\{\sqrt{\frac{\Delta_{j}(0)}{\Delta_{j}(t)}} \sqrt{\frac{\Delta_{0}(0)}{\Delta_{0}(t)}}\left(\frac{A_{j k}(t)}{A_{j k}(0)}-1\right)\right. \\
& \left.\quad-\sqrt{\frac{\Delta_{j}(0)}{\Delta_{j}(-t)}} \sqrt{\frac{\Delta_{0}(0)}{\Delta_{0}(-t)}}\left(\frac{A_{j k}(-t)}{A_{j k}(0)}-1\right)\right\} \mid \\
& \leq C \frac{\left|\gamma_{k}\right|^{2}}{k^{3}}\left(\sum_{|\alpha-k| \leq \frac{k}{2}}\left|\gamma_{\alpha}\right|^{2}+O\left(\frac{1}{k}\right)\right)
\end{aligned}
$$

and

$$
\begin{aligned}
& \mid \int_{0}^{1} \frac{d t}{\sqrt{1-t^{2}}}\left\{\sqrt{\frac{\Delta_{j}(0)}{\Delta_{j}(t)}} \sqrt{\frac{\Delta_{0}(0)}{\Delta_{0}(t)}}\left(\frac{A_{j k}(t)}{A_{j k}(0)}-1\right)\right. \\
& \left.\quad+\sqrt{\frac{\Delta_{j}(0)}{\Delta_{j}(-t)}} \sqrt{\frac{\Delta_{0}(0)}{\Delta_{0}(-t)}}\left(\frac{A_{j k}(-t)}{A_{j k}(0)}-1\right)\right\} \mid \\
& \leq C \frac{\left|\gamma_{k}\right|}{k^{3}}\left(\sum_{|\alpha-k| \leq \frac{k}{2}}\left|\gamma_{\alpha}\right|^{2}+O\left(\frac{1}{k}\right)\right) .
\end{aligned}
$$

Finally, using Lemma 2.3, we obtain

$$
\begin{aligned}
& \mid \frac{\gamma_{k}}{2} \int_{0}^{1} \frac{t d t}{\sqrt{1-t^{2}}}\left\{\sqrt{\frac{\Delta_{j}(0)}{\Delta_{j}(t)}} \sqrt{\frac{\Delta_{0}(0)}{\Delta_{0}(t)}} \frac{A_{j k}(t)}{A_{j k}(0)}\left(\frac{B_{j k}(t)}{B_{j k}(0)}-1\right)\right. \\
& \left.\quad-\sqrt{\frac{\Delta_{j}(0)}{\Delta_{j}(-t)}} \sqrt{\frac{\Delta_{0}(0)}{\Delta_{0}(-t)}} \frac{A_{j k}(-t)}{A_{j k}(0)}\left(\frac{B_{j k}(-t)}{B_{j k}(0)}-1\right)\right\} \mid
\end{aligned}
$$




$$
\leq C \frac{\left|\gamma_{k}\right|^{2}}{k^{3}}\left(\sum_{|\alpha-k| \leq \frac{k}{2}}\left|\gamma_{\alpha}\right|^{2}+O\left(\frac{1}{k^{3}}\right)\right)
$$

and

$$
\begin{aligned}
& \mid \int_{0}^{1} \frac{d t}{\sqrt{1-t^{2}}}\left\{\sqrt{\frac{\Delta_{j}(0)}{\Delta_{j}(t)}} \sqrt{\frac{\Delta_{0}(0)}{\Delta_{0}(t)}} \frac{A_{j k}(t)}{A_{j k}(0)}\left(\frac{B_{j k}(t)}{B_{j k}(0)}-1\right)\right. \\
& \left.\quad+\sqrt{\frac{\Delta_{j}(0)}{\Delta_{j}(-t)}} \sqrt{\frac{\Delta_{0}(0)}{\Delta_{0}(-t)}} \frac{A_{j k}(-t)}{A_{j k}(0)}\left(\frac{B_{j k}(-t)}{B_{j k}(0)}-1\right)\right\} \mid \\
& \leq C \frac{\left|\gamma_{k}\right|}{k^{3}}\left(\sum_{|\alpha-k| \leq \frac{k}{2}}\left|\gamma_{\alpha}\right|^{2}+O\left(\frac{1}{k^{3}}\right)\right) .
\end{aligned}
$$

We combine the above estimates to conclude

$$
\begin{aligned}
& \frac{\gamma_{k}}{2} \int_{0}^{1} \frac{t d t}{\sqrt{1-t^{2}}}\left(C_{j k}(t)-C_{j k}(-t)\right) \\
& =\frac{\gamma_{k}}{2} \int_{0}^{1} \frac{t d t}{\sqrt{1-t^{2}}}\left(\sqrt{\frac{\Delta_{j}(0)}{\Delta_{j}(t)}}-\sqrt{\frac{\Delta_{j}(0)}{\Delta_{j}(-t)}}\right) \\
& \quad-\frac{\gamma_{k}^{2}}{16} \frac{\pi}{\tau_{k}-\lambda_{0}}+\left|\gamma_{k}\right|^{2} O\left(\frac{1}{k^{3}}\right)\left(\sum_{|\alpha-k| \leq \frac{k}{2}}\left|\gamma_{\alpha}\right|^{2}+O\left(\frac{1}{k}\right)\right)
\end{aligned}
$$

and

$$
\begin{aligned}
& \int_{0}^{1} \frac{d t}{\sqrt{1-t^{2}}}\left(C_{j k}(t)+C_{j k}(-t)\right) \\
& =\int_{0}^{1} \frac{d t}{\sqrt{1-t^{2}}}\left(\sqrt{\frac{\Delta_{j}(0)}{\Delta_{j}(t)}}+\sqrt{\frac{\Delta_{j}(0)}{\Delta_{j}(-t)}}\right)+O\left(\frac{\left|\gamma_{k}\right|^{2}}{k^{2}}\right) \\
& \quad+O\left(\frac{\gamma_{k}}{k^{3}}\right)\left(\sum_{|\alpha-k| \leq k / 2}\left|\gamma_{\alpha}\right|^{2}+O\left(\frac{1}{k}\right)\right) \\
& =\pi\left(1-\left(\frac{1}{16} \frac{\gamma_{k}^{2}}{\Delta_{j}(0)}-\frac{3}{16} \frac{\gamma_{k}^{2}\left(\tau_{j}-\tau_{k}\right)^{2}}{\Delta_{j}(0)^{2}}\right)\right)+\frac{\gamma_{k}^{3}}{k^{3}} O(1)+O\left(\frac{\gamma_{k}^{2}}{k^{2}}\right) \\
& \quad+O\left(\frac{\gamma_{k}}{k^{3}}\right)\left(\sum_{|\alpha-k| \leq k / 2}\left|\gamma_{\alpha}\right|^{2}+O\left(\frac{1}{k}\right)\right) .
\end{aligned}
$$


Therefore, there exists $k_{0} \geq 1$ and a neighborhood $U_{q_{0}}$ of $q_{0}$ in $L_{0}^{2}\left(S^{1} ; \mathbb{C}\right)$ such that for $q$ in $U_{q_{0}}$, for any $k \geq k_{0}$ and $j \geq 1, \int_{0}^{1} \frac{d t}{\sqrt{1-t^{2}}}\left(C_{j k}(t)+C_{j k}(-t)\right) \neq 0$ and its inverse satisfies the estimate

$$
\begin{aligned}
\left(\int_{0}^{1} \frac{d t}{\sqrt{1-t^{2}}}\left(C_{j k}(t)+C_{j k}(-t)\right)\right)^{-1} \\
=\left(\int_{0}^{1} \frac{d t}{\sqrt{1-t^{2}}}\left(\sqrt{\frac{\Delta_{j}(0)}{\Delta_{j}(t)}}+\sqrt{\frac{\Delta_{j}(0)}{\Delta_{j}(-t)}}\right)\right)^{-1} \\
\\
\quad \cdot\left(1+O\left(\frac{\left|\gamma_{k}\right|^{2}}{k^{2}}\right)+O\left(\frac{\gamma_{k}}{k^{3}}\right)\left(\sum_{|\alpha-k| \leq k / 2}\left|\gamma_{\alpha}\right|^{2}+O\left(\frac{1}{k}\right)\right)\right) .
\end{aligned}
$$

Combining (2.34) and (2.36)

$$
\begin{aligned}
& \frac{\frac{\gamma_{k}}{2} \int_{0}^{1} \frac{t d t}{\sqrt{1-t^{2}}}\left(C_{j k}(t)-C_{j k}(-t)\right)}{\int_{0}^{1} \frac{d t}{\sqrt{1-t^{2}}}\left(C_{j k}(t)+C_{j k}(-t)\right)} \\
& =\frac{\frac{\gamma_{k}}{2} \int_{0}^{1} \frac{t d t}{\sqrt{1-t^{2}}}\left(\sqrt{\frac{\Delta_{j}(0)}{\Delta_{j}(t)}}-\sqrt{\frac{\Delta_{j}(0)}{\Delta_{j}(-t)}}\right)-\frac{\gamma_{k}^{2}}{16} \frac{\pi}{\tau_{k}-\lambda_{0}}+O\left(\frac{\left|\gamma_{k}\right|^{2}}{k^{3}}\right)\left(\sum_{|\alpha-k| \leq \frac{k}{2}}\left|\gamma_{\alpha}\right|^{2}+O\left(\frac{1}{k}\right)\right)}{\int_{0}^{1} \frac{d t}{\sqrt{1-t^{2}}}\left(\sqrt{\frac{\Delta_{j}(0)}{\Delta_{j}(t)}}+\sqrt{\frac{\Delta_{j}(0)}{\Delta_{j}(-t)}}\right)\left(1+O\left(\frac{\left|\gamma_{k}\right|^{2}}{k^{2}}\right)\right)} \\
& =\left\{-\frac{1}{8} \frac{\gamma_{k}^{2}}{\tau_{k}-\tau_{j}}-\frac{1}{16} \frac{\gamma_{k}^{2}}{\tau_{k}-\lambda_{0}}+\frac{\left|\gamma_{k}\right|^{2}}{k^{3}}\left(O\left(\left|\gamma_{k}\right|\right)+O\left(\left|\gamma_{j}\right|^{2}\right)\right)\right. \\
& \left.\quad+O\left(\frac{\left|\gamma_{k}\right|^{2}}{k^{3}}\right)\left(\sum_{|\alpha-k| \leq \frac{k}{2}}\left|\gamma_{\alpha}\right|^{2}+O\left(\frac{1}{k}\right)\right)\right\}\left\{1+O\left(\frac{\left|\gamma_{k}\right|^{2}}{k^{2}}\right)\right\} \\
& =-\frac{1}{8} \frac{\gamma_{k}^{2}}{\tau_{k}-\tau_{j}}-\frac{1}{16} \frac{\gamma_{k}^{2}}{\tau_{k}-\lambda_{0}}+O\left(\frac{\left|\gamma_{k}\right|^{2}}{k^{3}}\right)\left\{\left.\gamma_{k}|+| \gamma_{j}\right|^{2}+\sum_{|\alpha-k| \leq \frac{k}{2}}\left|\gamma_{\alpha}\right|^{2}\right\} \\
& \quad+\frac{\gamma_{k}^{4}}{\tau_{k}-\tau_{j}} O\left(\frac{1}{k^{2}}\right)+\gamma_{k}^{2} O\left(\frac{1}{k^{4}}\right) .
\end{aligned}
$$

\section{Frequencies of $\mathrm{KdV}$.}

The aim of this section is to prove Theorem 1, as stated in the introduction and to apply it to the initial value problem of $\mathrm{KdV}$. In the case where $q_{0} \in H_{0}^{1}\left(S^{1}\right)$, the frequencies $\omega_{j}=\frac{\partial \mathcal{H}}{\partial J_{j}}$ are well defined and can be obtained, 
using Riemann bilinear relations, from the expansion at infinity of the $L_{2}-$ integrable holomorphic differentials $\Omega_{j}=\frac{\varphi_{j}(\lambda) d \lambda}{\sqrt{\Delta(\lambda)^{2}-4}}$ constructed in Theorem 2.1. More explicitly, $\omega_{j}$ is given by (cf. e.g. $[\mathbf{E M}],[\mathbf{B K M}]$ )

$$
\omega_{j}=8 \pi\left(\int_{\Gamma_{j}} \frac{\varphi_{j}(\mu) d \mu}{\sqrt{\Delta(\mu)^{2}-4}}\right)^{-1}\left(\tau_{j}-\sum_{k \in \mathbb{N} \backslash\{j\}}\left(\mu_{k}^{(j)}-\tau_{k}\right)+\frac{\lambda_{0}}{2}\right)
$$

where $\Gamma_{j}$ is a counterclockwise oriented circle with center $\tau_{j}$ and sufficiently small radius $\rho_{j}>\left|\gamma_{j}\right| / 2$ so that all eigenvalues $\left(\lambda_{k}\right)_{k \in \mathbb{N} \backslash\{2 j, 2 j-1\}}$ lie outside $\Gamma_{j}$.

Due to Theorem 2.1, the right hand side of formula (3.1) is well defined even if $q_{0} \in L_{0}^{2}\left(S^{1}\right)$ and can be extended to an analytic function in a sufficiently small complex neighborhood $U_{q_{0}}$ of $q_{0}$ in $L_{0}^{2}\left(S^{1} ; \mathbb{C}\right)$. For $q$ in $U_{q_{0}}$ we therefore define the frequency $\omega_{j}$ by formula (3.1). In view of Theorem 2.1 we obtain the following

Theorem 3.1. Let $q_{0} \in L_{0}^{2}\left(S^{1}\right)$. Then there exists a neighborhood $U_{q_{0}}$ of $q_{0}$ in $L_{0}^{2}\left(S^{1} ; \mathbb{C}\right)$ so that, for any $j \geq 1$, the frequency $\omega_{j}$, given by $(3.1)$, is well defined and analytic.

It remains to prove the asymptotics for $\omega_{j}$ as $j \rightarrow \infty$ as stated in Theorem 1. We start by analyzing $\int_{\Gamma_{j}} \frac{\varphi_{j}(\mu) d \mu}{\sqrt{\Delta(u)^{2}-4}}$. Note that in the case where $\gamma_{j}=0$ we have by the residue theorem, taking into account the determination of the root,

$$
\int_{\Gamma_{j}} \frac{\varphi_{j}(\mu) d \mu}{\sqrt{\Delta(\mu)^{2}-4}}=\left.2 \pi \frac{\varphi_{j}\left(\tau_{j}\right)}{\sqrt{\frac{\Delta(\mu)^{2}-4}{\left(\tau_{j}-\mu\right)\left(\mu-\tau_{j}\right)}}}\right|_{\mu=\tau_{j}} .
$$

In the case where $\gamma_{j} \neq 0$, introduce $\mu(t)=\tau_{j}+\frac{\gamma_{j}}{2} t(-1 \leq t \leq 1)$ and

$$
\psi_{j}(t):=\frac{\varphi_{j}(\mu(t))}{\sqrt{\frac{\Delta(\mu(t))^{2}-4}{\left(\lambda_{2 j}-\mu(t)\right)\left(\mu(t)-\lambda_{2 j-1}\right)}}} .
$$

Then, with $\left(\lambda_{2 j}-\mu(t)\right)\left(\mu(t)-\lambda_{2 j-1}\right)=\frac{\gamma_{j}}{2}(1-t) \frac{\gamma_{j}}{2}(t+1)=\left(\frac{\gamma_{j}}{2}\right)^{2}\left(1-t^{2}\right)$

$$
\begin{aligned}
\int_{\Gamma_{j}} \frac{\varphi_{j}(\mu) d \mu}{\sqrt{\Delta(\mu)^{2}-4}} & =2 \int_{-1}^{1} \psi_{j}(t) \frac{\frac{\gamma_{j}}{2} d t}{\frac{\gamma_{j}}{2} \sqrt{1-t^{2}}} \\
& =2 \int_{0}^{1}\left(\psi_{j}(t)+\psi_{j}(-t)\right) \frac{d t}{\sqrt{1-t^{2}}} .
\end{aligned}
$$


Summarizing, we obtain

$$
\int_{\Gamma_{j}} \frac{\varphi_{j}(\mu) d \mu}{\sqrt{\Delta(\mu)^{2}-4}}= \begin{cases}2 \pi \psi_{j}(0) & \text { for } \gamma_{j}=0 \\ 2 \int_{0}^{1}\left(\psi_{j}(t)+\psi_{j}(-t)\right) \frac{d t}{\sqrt{1-t^{2}}} & \text { for } \gamma_{j} \neq 0\end{cases}
$$

The function $\psi_{j}(t)$ has a product representation

$$
\left(\prod_{k \neq j} \frac{\mu_{k}^{(j)}-\mu(t)}{k^{2} \pi^{2}}\right) \frac{1}{2}\left(\mu(t)-\lambda_{0}\right)^{-1 / 2} \prod_{k \neq j}\left(\frac{\left(\lambda_{2 k}-\mu(t)\right)\left(\lambda_{2 k-1}-\mu(t)\right)}{\left(k^{2} \pi^{2}\right)^{2}}\right)^{-1 / 2} .
$$

Notice that

$$
\begin{aligned}
\left(\lambda_{2 k}-\mu(t)\right)\left(\lambda_{2 k-1}-\mu(t)\right) & =\left(\tau_{k}-\mu(t)\right)^{2}-\left(\frac{\gamma_{k}}{2}\right)^{2} \\
& =\left(\tau_{k}-\mu(t)\right)^{2}\left(1-\frac{\left(\gamma_{k} / 2\right)^{2}}{\left(\tau_{k}-\mu(t)\right)^{2}}\right)
\end{aligned}
$$

and, with $\xi_{k}^{(j)}=\mu_{k}^{(j)}-\tau_{k}$,

$$
\mu_{k}^{(j)}-\mu(t)=\left(\tau_{k}-\mu(t)\right)\left(1+\frac{\xi_{k}^{(j)}}{\tau_{k}-\mu(t)}\right)
$$

Therefore,

$$
2 \psi_{j}(t)=\left(\mu(t)-\lambda_{0}\right)^{-1 / 2} \prod_{k \neq j}\left(1+\frac{\xi_{k}^{(j)}}{\tau_{k}-\mu(t)}\right) \prod_{k \neq j}\left(1-\frac{\left(\gamma_{k} / 2\right)^{2}}{\left(\tau_{k}-\mu(t)\right)^{2}}\right)^{-1 / 2} .
$$

Similarly, as in Section 2, it is convenient to introduce

$$
\begin{aligned}
\Delta_{0}(t) & :=\mu(t)-\lambda_{0} ; \\
A_{j}(t) & :=\prod_{k \neq j}\left(1+\frac{\xi_{k}^{(j)}}{\tau_{k}-\mu(t)}\right) \\
B_{j}(t) & :=\prod_{k \neq j}\left(1-\frac{\left(\gamma_{k} / 2\right)^{2}}{\left(\tau_{k}-\mu(t)\right)^{2}}\right)^{-1 / 2} .
\end{aligned}
$$

This allows us to write $\psi_{j}(t)$ as follows

$$
2 \psi_{j}(t)=\Delta_{0}(t)^{-1 / 2} A_{j}(t) B_{j}(t) .
$$


Further, for some $\theta_{ \pm}(t)$ with $-t<\theta_{-}(t)<\theta_{+}(t)<t$

$$
2\left(\psi(t)+\psi_{j}(-t)\right)=4 \psi_{j}(0)\left(1+\frac{\psi_{j}^{\prime \prime}\left(\theta_{+}(t)\right)-\psi_{j}^{\prime \prime}\left(\theta_{-}(t)\right)}{2 \psi_{j}(0)} \frac{t^{2}}{2}\right) .
$$

Lemma 3.2. There exists $C>0$ such that, for $q$ in $U_{q_{0}}$ and $j \geq 1$,

$$
\begin{aligned}
& \mid\left(2 \psi_{j}(0)\right)^{-1}-\left(\tau_{j}-\lambda_{0}\right)^{1 / 2}\left\{1-\sum_{k \neq j} \frac{\xi_{k}^{(j)}}{\tau_{k}-\tau_{j}}\right. \\
& \left.\quad-\frac{1}{2} \sum_{k \neq j} \frac{\left(\gamma_{k} / 2\right)^{2}}{\left(\tau_{k}-\tau_{j}\right)^{2}}+\frac{1}{2}\left(\sum_{k \neq j} \frac{\xi_{k}^{(j)}}{\tau_{k}-\tau_{j}}\right)^{2}+\frac{1}{2} \sum_{k \neq j}\left(\frac{\xi_{k}^{(j)}}{\tau_{k}-\tau_{j}}\right)^{2}\right\} \mid \\
& \leq \frac{C}{j^{3}}\left(\sum_{k \geq j / 2}\left|\gamma_{k}\right|^{2}\right)^{2}+\frac{C}{j^{5}} .
\end{aligned}
$$

Proof. Notice that $\mu(0)=\tau_{j}$ and therefore

$$
\begin{aligned}
& \left(2 \psi_{j}(0)\right)^{-1} \\
& =\left(\tau_{j}-\lambda_{0}\right)^{1 / 2} \prod_{k \neq j}\left(1+\frac{\xi_{k}^{(j)}}{\left(\tau_{k}-\tau_{j}\right)}\right)^{-1} \prod_{k \neq j}\left(1-\frac{\left(\gamma_{k} / 2\right)^{2}}{\left(\tau_{k}-\tau_{j}\right)^{2}}\right)^{1 / 2} .
\end{aligned}
$$

Further

$$
\begin{aligned}
\prod_{k \neq j}\left(1+\frac{\xi_{k}^{(j)}}{\left(\tau_{k}-\tau_{j}\right)}\right)^{-1}= & 1-\sum_{k \neq j} \frac{\xi_{k}^{(j)}}{\tau_{k}-\tau_{j}}+\sum_{\substack{k_{1}, k_{2} \neq j \\
k_{1}<k_{2}}} \frac{\xi_{k_{1}}^{(j)}}{\tau_{k_{1}}-\tau_{j}} \frac{\xi_{k_{2}}^{(j)}}{\tau_{k_{2}}-\tau_{j}} \\
& +\sum_{k \neq j} \frac{\left(\xi_{k}^{(j)}\right)^{2}}{\left(\tau_{k}-\tau_{j}\right)^{2}}+O\left(\frac{1}{j^{6}}\right)
\end{aligned}
$$

where for the estimate of the error term we used that, for $|k-j| \geq j / 2$

$$
\frac{1}{\left|\tau_{k}-\tau_{j}\right|}=O\left(\frac{1}{j^{2}}\right)
$$

and for $|k-j| \leq \frac{j}{2}$

$$
\left|\xi_{k}^{(j)}\right|=\frac{\left|\gamma_{k}\right|^{2}}{k} O(1)=O\left(\frac{\left|\gamma_{k}\right|^{2}}{j}\right) \quad \text { and } \quad \frac{1}{\left|\tau_{k}-\tau_{j}\right|}=O\left(\frac{1}{j}\right)
$$


to conclude that

$$
\left|\frac{\xi_{k}^{(j)}}{\tau_{k}-\tau_{j}}\right| \leq\left|\gamma_{k}\right|^{2} O\left(\frac{1}{j^{2}}\right)
$$

Similarly

$$
\begin{aligned}
& \prod_{k \neq j}\left(1-\frac{\left(\gamma_{k} / 2\right)^{2}}{\left(\tau_{k}-\tau_{j}\right)^{2}}\right)^{1 / 2} \\
& =1-\frac{1}{2} \sum_{k \neq j} \frac{\left(\gamma_{k} / 2\right)^{2}}{\left(\tau_{k}-\tau_{j}\right)^{2}}-\frac{1}{8} \sum_{k \neq j} \frac{\left(\gamma_{k} / 2\right)^{4}}{\left(\tau_{k}-\tau_{j}\right)^{4}} \\
& \quad+\frac{1}{4} \sum_{\substack{k_{1}, k_{2} \neq j \\
k_{1}<k_{2}}} \frac{\left(\gamma_{k_{1}} / 2\right)^{2}\left(\gamma_{k_{2}} / 2\right)^{2}}{\left(\tau_{k_{1}}-\tau_{j}\right)^{2}\left(\tau_{k_{2}}-\tau_{j}\right)^{2}}+O\left(\frac{1}{j^{6}}\right) \\
& =1-\frac{1}{2} \sum_{k \neq j} \frac{\left(\gamma_{k} / 2\right)^{2}}{\left(\tau_{k}-\tau_{j}\right)^{2}}+O\left(\frac{1}{j^{4}}\right) \sum_{k \geq j / 2} \gamma_{k}^{4} \\
& \quad+O\left(\frac{1}{j^{4}}\right) \sum_{\substack{k_{1}<k_{2} \\
k_{2}, k_{1} \geq j / 2}} \gamma_{k_{1}}^{2} \gamma_{k_{2}}^{2}+O\left(\frac{1}{j^{6}}\right) .
\end{aligned}
$$

Combining (3.11)-(3.14) we obtain

$$
\begin{aligned}
& \left(2 \psi_{j}(0)\right)^{-1} \\
& =\left(\tau_{j}-\lambda_{0}\right)^{1 / 2} \cdot\left\{1-\sum_{k \neq j} \frac{\xi_{k}^{(j)}}{\tau_{k}-\tau_{j}}-\frac{1}{2} \sum_{k \neq j} \frac{\left(\gamma_{k} / 2\right)^{2}}{\left(\tau_{k}-\tau_{j}\right)^{2}}\right. \\
& \left.\quad+\frac{1}{2}\left(\sum_{k \neq j} \frac{\xi_{k}^{(j)}}{\tau_{k}-\tau_{j}}\right)^{2}+\frac{1}{2} \sum_{k \neq j}\left(\frac{\xi_{k}^{(j)}}{\tau_{k}-\tau_{j}}\right)^{2}+R_{j}\right\}
\end{aligned}
$$

where, with $\left(\sum_{k \neq j} \frac{\xi_{k}^{(j)}}{\tau_{k}-\tau_{j}}\right)\left(\sum_{k \neq j} \frac{\left(\gamma_{k} / 2\right)^{2}}{\left(\tau_{k}-\tau_{j}\right)^{2}}\right)=O\left(\frac{1}{j^{4}}\right)\left(\sum_{k \geq j / 2}\left|\gamma_{k}^{2}\right|\right)^{2}+O\left(\frac{1}{j^{6}}\right)$, we have

$$
R_{j}:=O\left(\frac{1}{j^{4}}\right) \sum_{k \geq j / 2} \gamma_{k}^{4}+O\left(\frac{1}{j^{4}}\right) \sum_{\substack{k_{1} \geq j / 2 \\ k_{2} \geq j / 2}} \gamma_{k_{1}}^{2} \gamma_{k_{2}}^{2}+O\left(\frac{1}{j^{6}}\right) .
$$

Finally use that $\frac{1}{2}\left(\tau_{j}-\lambda_{0}\right)^{1 / 2}=\frac{j \pi}{2}+O\left(\frac{1}{j^{2}}\right)$ to obtain (3.10).

\section{Lemma 3.3.}

$$
\sup _{0 \leq|t| \leq 1} \frac{\left|\frac{d^{2}}{d t^{2}} \psi_{j}(t)\right|}{\psi_{j}(0)} \leq C \frac{\left|\gamma_{j}\right|^{2}}{j^{4}}
$$


Proof. In a straightforward fashion one computes

$$
\begin{aligned}
\frac{d}{d t} \psi_{j}(t)= & \frac{\gamma_{j}}{2} \psi_{j}(t)\left\{\frac{-1 / 2}{\tau_{j}-\lambda_{0}+\frac{\gamma_{j}}{2} t}+\sum_{k \neq j} \frac{1}{1+\frac{\xi_{k}^{(j)}}{\tau_{k}-\mu(t)}} \frac{(-1) \xi_{k}^{(j)}}{\left(\tau_{k}-\mu(t)\right)^{2}}(-1)\right. \\
& \left.+\sum_{k \neq j} \frac{(-1 / 2)}{1-\frac{\left(\gamma_{k} / 2\right)^{2}}{\left(\tau_{k}-\mu(t)\right)^{2}}} \frac{(-1)(-2)\left(\gamma_{k} / 2\right)^{2}}{\left(\tau_{k}-\mu(t)\right)^{3}}(-1)\right\} \\
= & \frac{\gamma_{j}}{2} \psi_{j}(t)\left\{\frac{-1 / 2}{\tau_{j}-\lambda_{0}+\frac{\gamma_{j}}{2} t}+\sum_{k \neq j} \frac{1}{1+\frac{\xi_{k}^{(j)}}{\left(\tau_{k}-\mu(t)\right)}} \frac{\xi_{k}^{(j)}}{\left(\tau_{k}-\mu(t)\right)^{2}}\right. \\
& \left.+\sum_{k \neq j} \frac{1}{1-\frac{\left(\gamma_{k} / 2\right)^{2}}{\left(\tau_{k}-\mu(t)\right)^{2}}} \frac{\left(\gamma_{k} / 2\right)^{2}}{\left(\tau_{k}-\mu(t)\right)^{3}}\right\}
\end{aligned}
$$

$$
\begin{aligned}
\frac{d^{2}}{d t^{2}} \psi_{j}(t)= & \frac{1}{\psi_{j}(t)}\left(\frac{d}{d t} \psi_{j}(t)\right)^{2}+\left(\frac{\gamma_{j}}{2}\right)^{2} \psi_{j}(t) \\
& \cdot\left\{\frac{1 / 2}{\left(\tau_{j}-\lambda_{0}+\frac{\gamma_{j}}{2} t\right)^{2}}+\sum_{k \neq j} \frac{-1}{\left(1+\frac{\xi_{k}^{(j)}}{\tau_{k}-\mu(t)}\right)^{2}}\left(\frac{\xi_{k}^{(j)}}{\left(\tau_{k}-\mu(t)\right)^{2}}\right)^{2}\right. \\
& +\sum_{k \neq j} \frac{1}{1+\frac{\xi_{k}^{(j)}}{\left(\tau_{k}-\mu(t)\right)^{2}}} \frac{2 \xi_{k}^{(j)}}{\left(\tau_{k}-\mu(t)\right)^{3}} \\
& +\sum_{k \neq j} \frac{(-1)}{\left(1-\frac{\left(\gamma_{k} / 2\right)^{2}}{\left(\tau_{k}-\mu(t)\right)^{2}}\right)^{2}}\left(\frac{\left(\gamma_{k} / 2\right)^{2}}{\left(\tau_{k}-\mu(t)\right)^{3}}\right)^{2}(-1) \\
& \left.+\sum_{k \neq j} \frac{1}{1-\frac{\left(\gamma_{k} / 2\right)^{2}}{\left(\tau_{k}-\mu(t)\right)^{2}}} \frac{(-3)\left(\gamma_{k} / 2\right)^{2}}{\left(\tau_{k}-\mu(t)\right)^{4}}(-1)\right\}
\end{aligned}
$$

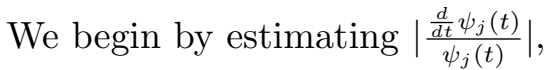

$$
\left|\frac{\frac{d}{d t} \psi_{j}(t)}{\psi_{j}(t)}\right| \leq C\left|\gamma_{j}\right|\left\{\frac{1}{j^{2}}+\sum_{k \neq j}\left|\frac{\xi_{k}^{(j)}}{\tau_{k}-\mu(t)}\right| \frac{1}{\left|\tau_{k}-\mu(t)\right|}+\sum_{k \neq j} \frac{\left|\gamma_{k}\right|^{2}}{\left|\tau_{k}-\mu(t)\right|^{3}}\right\}
$$




$$
\leq C \frac{\left|\gamma_{j}\right|}{j^{2}}
$$

Therefore,

$$
\sup _{|t| \leq 1}\left|\frac{\left(\frac{d}{d t} \psi_{j}(t)\right)^{2}}{\psi_{j}(0) \psi_{j}(t)}\right| \leq C \frac{\left|\gamma_{j}\right|^{2}}{j^{4}}
$$

All the other terms are treated similarly.

Theorem 3.4. Let $q_{0} \in L_{0}^{2}\left(S^{1}\right)$. Then there exists a neighborhood $U_{q_{0}}$ of $q_{0}$ in $L_{0}^{2}\left(S^{1} ; \mathbb{C}\right)$ and $C>0$ so that for any $q$ in $U_{q_{0}}$ and $j \geq 1$,

$$
\left|\left(\int_{\Gamma_{j}} \frac{\varphi_{j}(\mu) d \mu}{\sqrt{\Delta(\mu)^{2}-4}}\right)^{-1}-\left(2 \pi \psi_{j}(0)\right)^{-1}\right| \leq C \frac{\left|\gamma_{j}\right|^{2}}{j^{3}}
$$

and

$$
\begin{aligned}
& \left|\left(2 \psi_{j}(0)\right)^{-1}-\left\{\tau_{j}^{1 / 2}-\frac{1}{2} \frac{\lambda_{0}}{\tau_{j}^{1 / 2}}-\tau_{j}^{1 / 2} \sum_{k \leq j / 2} \frac{\xi_{k}^{(j)}}{\tau_{k}-\tau_{j}}\right\}\right| \\
& \leq \frac{C}{j^{2}}\left(\sum_{k \geq j / 2}\left|\gamma_{k}\right|^{2}+\frac{1}{j}\right) .
\end{aligned}
$$

Proof. Notice that, according to (3.5), (3.9) and as $\int_{0}^{1} \frac{d t}{\sqrt{1-t^{2}}}=\frac{\pi}{2}$

$$
\begin{aligned}
& \left(\int_{\Gamma_{j}} \frac{\varphi_{j}(\mu) d \mu}{\sqrt{\Delta(\mu)^{2}-4}}\right)^{-1} \\
& =\left(2 \pi \psi_{j}(0)\right)^{-1}\left(1+\int_{0}^{1} \frac{\psi_{j}^{\prime \prime}\left(\theta_{+}(t)\right)-\psi_{j}^{\prime \prime}\left(\theta_{-}(t)\right)}{4 \pi \psi_{j}(0)} \frac{t^{2} d t}{\sqrt{1-t^{2}}}\right)^{-1} .
\end{aligned}
$$

Thus, by Lemma 3.3

$$
\begin{aligned}
\left|\left(\int_{\Gamma_{j}} \frac{\varphi_{j}(\mu) d \mu}{\sqrt{\Delta(\mu)^{2}-4}}\right)^{-1}-\left(2 \pi \psi_{j}(0)\right)^{-1}\right| & \leq \frac{C}{\left|\psi_{j}(0)\right|} \sup _{|t| \leq 1}\left|\frac{\psi_{j}^{\prime \prime}(t)}{\psi_{j}(0)}\right| \\
& \leq C j \frac{\left|\gamma_{j}\right|^{2}}{j^{4}} \leq C \frac{\left|\gamma_{j}\right|^{2}}{j^{3}}
\end{aligned}
$$

This proves (3.20). To prove the estimates (3.21) use (3.10) and notice that

$$
\left|\left(\tau_{j}-\lambda_{0}\right)^{1 / 2} \sum_{k \leq j / 2} \frac{\frac{1}{2}\left(\gamma_{k} / 2\right)^{2}}{\left(\tau_{k}-\tau_{j}\right)^{2}}\right| \leq C j\left(\sum_{k \leq j / 2}\left|\gamma_{k}\right|^{2}\right) \frac{1}{j^{4}}=O\left(\frac{1}{j^{3}}\right)
$$


and, in view of (3.13),

$$
\left|\left(\tau_{j}-\lambda_{0}\right)^{1 / 2}\left(\sum_{k \neq j}\left|\frac{\xi_{k}^{(j)}}{\tau_{k}-\tau_{j}}\right|\right)^{2}\right| \leq C j\left(\sum_{k} \frac{\left|\gamma_{k}\right|^{2}}{j^{2}}\right)^{2}=O\left(\frac{1}{j^{3}}\right) .
$$

According to Theorem 2.7

$$
\begin{aligned}
& \left|\left(\tau_{j}-\lambda_{0}\right)^{1 / 2} \sum_{k \geq j / 2}\left(\frac{\xi_{k}^{(j)}}{\tau_{k}-\tau_{j}}+\frac{\frac{1}{2}\left(\gamma_{k} / 2\right)^{2}}{\left(\tau_{k}-\tau_{j}\right)^{2}}\right)\right| \\
& \leq C j \sum_{k \geq j / 2} \frac{\left|\gamma_{k}\right|^{2}}{\left|\tau_{k}-\tau_{j}\right|\left|\tau_{k}-\lambda_{0}\right|}+C j \sum_{k \geq j / 2} \frac{\left|\gamma_{k}\right|^{2}}{j^{3}\left|\tau_{k}-\tau_{j}\right|} \\
& =\frac{C}{j^{2}} \sum_{k \geq j / 2}\left|\gamma_{k}\right|^{2}+O\left(\frac{1}{j^{3}}\right) .
\end{aligned}
$$

Finally

$$
\begin{aligned}
\left(\tau_{j}-\lambda_{0}\right)^{1 / 2} & =\tau_{j}^{1 / 2}\left(1-\frac{\lambda_{0}}{\tau_{j}}\right)^{1 / 2}=\tau_{j}^{1 / 2}\left(1-\frac{1}{2} \frac{\lambda_{0}}{\tau_{j}}+O\left(\frac{1}{j^{4}}\right)\right) \\
& =\tau_{j}^{1 / 2}-\frac{1}{2} \frac{\lambda_{0}}{\tau_{j}^{1 / 2}}+O\left(\frac{1}{j^{3}}\right) .
\end{aligned}
$$

Combining these estimates we obtain

$$
\begin{aligned}
& \left(2 \psi_{j}(0)\right)^{-1}=\left(\tau_{j}^{1 / 2}-\frac{1}{2} \frac{\lambda_{0}}{\tau_{j}^{1 / 2}}+0\left(\frac{1}{j^{3}}\right)\right) \cdot\left(1-\sum_{k \leq j / 2} \frac{\xi_{k}^{(j)}}{\tau_{k}-\tau_{j}}\right) \\
& +O\left(\frac{1}{j^{2}}\right)\left(\sum_{k \geq j / 2}\left|\gamma_{k}\right|^{2}+\frac{1}{j}\right) \\
& =\tau_{j}^{1 / 2}-\frac{1}{2} \frac{\lambda_{0}}{\tau_{j}^{1 / 2}}-\tau_{j}^{1 / 2} \sum_{k \leq j / 2} \frac{\xi_{k}^{(j)}}{\tau_{k}-\tau_{j}}+O\left(\frac{1}{j^{3}}\right) \\
& +O\left(\frac{1}{j^{2}}\right)\left(\sum_{k \geq j / 2}\left|\gamma_{k}\right|^{2}+\frac{1}{j}\right)
\end{aligned}
$$

which proves (3.21).

Combining Theorem 3.4 and Theorem 2.7 we obtain the following result which completes the proof of Theorem 1 : 
Theorem 3.5. Let $q_{0} \in L_{0}^{2}\left(S^{1}\right)$. Then there exists a neighborhood $U_{q_{0}}$ of $q_{0}$ in $L_{0}^{2}\left(S^{1} ; \mathbb{C}\right)$ and $C>0$ so that for any $q$ in $U_{q_{0}}$ and $j \geq 1$,

$$
\left|\omega_{j}-(2 \pi j)^{3}\right| \leq C\left(\sum_{k \geq j / 2}\left|\gamma_{k}\right|^{2}+\left(\sqrt{\tau_{j}}-j \pi\right) j^{2} \pi^{2}+O\left(\frac{1}{j}\right)\right) .
$$

Proof. According to (3.1) and using (3.20)

$$
\begin{aligned}
\omega_{j}= & 8 \pi\left(\int_{\Gamma_{j}} \frac{\varphi_{j}(\mu) d \mu}{\sqrt{\Delta(\mu)^{2}-4}}\right)^{-1}\left(\tau_{j}-\sum_{k \in \mathbb{N} \backslash\{j\}}\left(\mu_{k}^{(j)}-\tau_{k}\right)+\frac{\lambda_{0}}{2}\right) \\
= & 8 \pi\left(\left(2 \pi \psi_{j}(0)\right)^{-1}\right)\left\{\tau_{j}-\sum_{k \leq j / 2}\left(\mu_{k}^{(j)}-\tau_{k}\right)+\frac{\lambda_{0}}{2}\right\}+O\left(\sum_{k \geq j}\left|\gamma_{k}\right|^{2}\right) \\
& +O\left(\frac{1}{j}\right) .
\end{aligned}
$$

By (3.21),

$$
\begin{aligned}
\omega_{j}= & \frac{8 \pi}{\pi}\left(\tau_{j}^{1 / 2}-\frac{1}{2} \frac{\lambda_{0}}{\tau_{j}^{1 / 2}}-\tau_{j}^{1 / 2} \sum_{k \leq j / 2} \frac{\xi_{k}^{(j)}}{\tau_{k}-\tau_{j}}\right)\left(\tau_{j}-\sum_{k \leq j / 2} \xi_{k}^{(j)}+\frac{\lambda_{0}}{2}\right) \\
& +O(1)\left(\sum_{k \geq j}\left|\gamma_{k}\right|^{2}+\frac{1}{j}\right) \\
= & 8\left\{\tau_{j}^{3 / 2}-\frac{1}{2} \lambda_{0} \tau_{j}^{1 / 2}-\tau_{j}^{3 / 2} \sum_{k \leq j / 2} \frac{\xi_{k}^{(j)}}{\tau_{k}-\tau_{j}}-\tau_{j}^{1 / 2} \sum_{k \leq j / 2} \xi_{k}^{(j)}+\frac{1}{2} \lambda_{0} \tau_{j}^{1 / 2}\right\} \\
& +O(1)\left(\sum_{k \geq j}\left|\gamma_{k}\right|^{2}+\frac{1}{j}\right) .
\end{aligned}
$$

Notice that

$$
\begin{aligned}
& \left|-\tau_{j}^{3 / 2} \sum_{k \leq j / 2} \frac{\xi_{k}^{(j)}}{\tau_{k}-\tau_{j}}-\tau_{j}^{1 / 2} \sum_{k \leq j / 2} \xi_{k}^{(j)}\right| \\
& =\left|\tau_{j}^{1 / 2} \sum_{k \leq j / 2} \xi_{k}^{(j)}\left(\frac{\tau_{j}}{\tau_{j}-\tau_{k}}-1\right)\right| \\
& \leq C|j| \sum_{k \leq j / 2}\left|\xi_{k}^{(j)}\right|\left|\frac{\tau_{k}}{\tau_{j}-\tau_{k}}\right|
\end{aligned}
$$




$$
\leq C \frac{1}{j} \sum_{k \leq j / 2} k^{2}\left|\xi_{k}^{(j)}\right|=O\left(\frac{1}{j}\right)
$$

where we used that, by Theorem 2.7, $\sum_{k \leq j / 2} k^{2}\left|\xi_{k}^{(j)}\right| \leq C$.

Finally, write $\sqrt{\tau_{j}}=j \pi+\frac{\hat{\tau_{j}}}{(j \pi)^{2}}$, to obtain (with $\hat{\tau}_{j} \in l^{2}(j)$ )

$$
\begin{aligned}
\tau_{j}^{3 / 2} & =\left((j \pi)^{2}+2 \frac{\hat{\tau}_{j}}{(j \pi)}+O\left(\frac{1}{j^{4}}\right)\right)\left(j \pi+\frac{\hat{\tau}_{j}}{(j \pi)^{2}}\right) \\
& =(j \pi)^{3}+3 \hat{\tau}_{j}+O\left(\frac{1}{j^{3}}\right) .
\end{aligned}
$$

Combining (3.27)-(3.29) leads to the claimed estimate.

The above results can be applied to the initial value problem for $K d V$,

$$
\begin{aligned}
\partial_{t} v: & =-\partial_{x}^{3} v+3 \partial_{x}\left(v^{2}\right) \quad\left(t>0 ; x \in S^{1}\right) \\
v(x, 0) & =q(x) .
\end{aligned}
$$

Recently, using a fixed point argument, Bourgain [Bo1] has constructed weak solutions $v_{B}(x, t)=\mathcal{S}_{B}(q)(x, t)$ of (3.30)-(3.31) for initial data in $L^{2}\left(S^{1}\right)$ globally in time and proved that these solutions are unique within a certain space of functions $f: \mathbb{R} \times[-T, T] \rightarrow \mathbb{R}$ (and $T>0$ ) and that they can be approximated within this space by smooth solutions corresponding to a smooth approximation of the initial data.

We obtain Bourgain's weak solutions $\mathcal{S}_{B}(q)$ of (3.30)-(3.31) by using action-angle coordinates as follows: Given $q$ in $L^{2}\left(S^{1}\right)$, define $p:=q-[q] \in$ $L_{0}^{2}\left(S^{1}\right)$ where $[q]=\int_{S^{1}} q(x) d x$ and introduce $(j \geq 1)$, with $\omega_{j}(p)$ given by Theorem 1,

$$
\omega_{j}(q)=\omega_{j}(p)+12[q] \pi j .
$$

In view of Theorem 3.1, the $\omega_{j}$ 's are real analytic functions on $L^{2}\left(S^{1}\right)$. Denote by $\Omega: L_{0}^{2}\left(S^{1}\right) \rightarrow \ell_{1 / 2}^{2}\left(\mathbb{R}^{2}\right)$ the symplectomorphism $\Omega(p)=(x(p), y(p))=$ $\left(x_{j}(p), y_{j}(p)\right)_{j \geq 1}$ constructed in [BBGK] and denote by $J_{j}, \alpha_{j}$ the symplectic polar coordinates corresponding to $x_{j}, y_{j}$, i.e.

$$
\left(x_{j}, y_{j}\right)=\left(\sqrt{2 J_{j}} \cos \alpha_{j}, \sqrt{2 J_{j}} \sin \alpha_{j}\right) .
$$

Define $(x(t), y(t)) \equiv(x(t, q), y(t, q))$ by setting

$$
(x(t), y(t))=\left(\sqrt{2 J_{j}} \cos \left(\omega_{j}(q) t+\alpha_{j}\right), \sqrt{2 J_{j}} \sin \left(\omega_{j}(q) t+\alpha_{j}\right)\right)_{j \geq 1}
$$


and introduce the solution operator $\mathcal{S}^{(1)}$

$$
\mathcal{S}^{(1)}: L^{2}\left(S^{1}\right) \rightarrow C\left(\mathbb{R} ; L^{2}\left(S^{1}\right)\right)
$$

defined by

$$
\mathcal{S}^{(1)}(q)(t)=[q]+\Omega^{-1}(x(t), y(t)) .
$$

To prove that (3.35) defines a weak solution of $K d V$, we approximate the initial data $q$ by finite gap potentials $\left(q_{N}\right)_{N \geq 1}$ with

$$
q_{N}:=\Omega^{-1}\left(\Pi_{N}(\Omega(p))\right)+[q]
$$

where $\Pi_{N}$ denotes the projection

$$
\Pi_{N}: \ell_{1 / 2}^{2}\left(\mathbb{R}^{2}\right) \rightarrow \ell_{1 / 2}^{2}\left(\mathbb{R}^{2}\right),\left(x_{j}, y_{j}\right)_{j \geq 1} \mapsto\left(\left(x_{j}, y_{j}\right)_{1 \leq j \leq N}, 0\right) .
$$

Note that $q=\lim _{N \rightarrow \infty} q_{N}$ in $L^{2}\left(S^{1}\right)$ and that for any $j \geq 1, \omega_{j}(q)=$ $\lim _{N \rightarrow \infty} \omega_{j}\left(q_{N}\right)$ uniformly in $j$. Therefore

$$
(x(\cdot, q), y(\cdot, q))=\lim _{N \rightarrow \infty}\left(x\left(\cdot, q_{N}\right), y\left(\cdot, q_{N}\right)\right)
$$

in $C\left([-T, T], \ell_{1 / 2}^{2}\left(\mathbb{R}^{2}\right)\right)$ (for any $T>0$ ), which in turn implies that

$$
\mathcal{S}^{(1)}(q)=\lim _{N \rightarrow \infty} \mathcal{S}^{(1)}\left(q_{N}\right)\left(\text { in } C\left([-T, T] ; L^{2}\left(S^{1}\right)\right)\right) .
$$

Notice that for arbitrary $N \geq 1, \mathcal{S}^{(1)}\left(q_{N}\right)(t)$ is an $N$-gap potential for any $t$ and therefore a classical solution of $K d V$ with initial data $q_{N}$. For $c \in \mathbb{R}$, we have introduced the symplectic leaf $L_{c}^{2}\left(S^{1}\right)=\left\{q \in L^{2}\left(S^{1}\right) ; \int_{S^{1}} q(x) d x=c\right\}$.

Theorem 3.6. Let $c \in \mathbb{R}$. Then

(i) $\mathcal{S}^{(1)}(q)=\mathcal{S}_{B}(q)$ for $q$ in $L_{c}^{2}\left(S^{1}\right)$.

(ii) Given $q_{1}, q_{2}$ in $L_{c}^{2}\left(S^{1}\right)$ there exists $M>0$ so that for any $t \in \mathbb{R}$,

$$
\left\|\left(\mathcal{S}^{(1)} q_{1}\right)(\cdot, t)-\left(\mathcal{S}^{(1)} q_{2}\right)(\cdot, t)\right\|_{L^{2}\left(S^{1}\right)} \leq M(1+|t|)\left\|q_{1}-q_{2}\right\|_{L^{2}\left(S^{1}\right)} .
$$

(iii) For any $T>0, \mathcal{S}^{(1)}: L_{c}^{2}\left(S^{1}\right) \rightarrow C\left([-T, T] ; L_{c}^{2}\left(S^{1}\right)\right)$ is real analytic.

For remarks concerning results related to Theorem 3.6 we refer the reader to the introduction.

Proof of Theorem 3.6. Let $q$ be in $L^{2}\left(S^{1}\right)$ and $q_{N}(N \geq 1)$ be defined as in (3.36). As classical solutions of $K d V$ are unique we conclude that $\mathcal{S}^{(1)}\left(q_{N}\right)=$ $\mathcal{S}_{B}\left(q_{N}\right)$ for any $N \geq 1$. Moreover Bourgain proved that $\lim _{N \rightarrow \infty} \mathcal{S}_{B}\left(q_{N}\right)=$ 
$\mathcal{S}_{B}(q)$ in $C\left([-T, T] ; L^{2}\left(S^{1}\right)\right)$. Together with (3.38) we conclude that $\mathcal{S}^{(1)}(q)=$ $\mathcal{S}_{B}(q)$.

Statements (ii) and (iii) rely both on Theorem 3.5 which, combined with (3.32) leads to the following result: Let $c \in \mathbb{R}$ and $q_{0} \in L_{c}^{2}\left(S^{1}\right)$. Then there exists a neighborhood $U_{q_{0}}$ of $q_{0}$ in $L_{c}^{2}\left(S^{1} ; \mathbb{C}\right)$ so that

$$
\omega_{j}(q) \text { can be analytically extended to } U_{q_{0}}
$$

and

$$
\omega_{j}(q)=(2 \pi j)^{3}+12 c \pi j+o(1) \quad(\text { as } j \rightarrow \infty)
$$

uniformly for $q$ in $U_{q_{0}}$.

Fix $0<T<\infty$ arbitrarily. By choosing $U_{q_{0}}$ smaller, if necessary, we can assume that $\Omega$ can be analytically extended to $\left\{\mathcal{S}^{(1)}(q)(t)-c ; q \in U ;|t| \leq T\right\}$ and that its inverse is analytic as well. Further note that $\ell_{1 / 2}^{2}\left(C\left([-T, T] ; \mathbb{C}^{2}\right)\right)$ can be embedded into $C\left([-T, T] ; \ell_{1 / 2}^{2}\left(\mathbb{C}^{2}\right)\right)$, the complexification of the Banach space $C\left([-T, T] ; \ell_{1 / 2}^{2}\left(\mathbb{R}^{2}\right)\right)$. It therefore suffices to prove that $\widetilde{\widetilde{\mathcal{S}}}^{(1)}$ : $U_{1} \rightarrow \ell_{1 / 2}^{2}\left(C\left([-T, T] ; \mathbb{C}^{2}\right)\right)$ is analytic where $U_{1}=\Omega\left(U_{q_{0}}-c\right)$ and where, with $\widetilde{\widetilde{\omega}}_{j}=\omega_{j}\left(\Omega^{-1}(x, y)\right)+12 c \pi j($ cf. $(3.32))$,

$$
\begin{aligned}
& \widetilde{\widetilde{\mathcal{S}}}^{(1)}\left(\left(\sqrt{2 J_{j}} \cos \alpha_{j}, \sqrt{2 J_{j}} \sin \alpha_{j}\right)_{j \geq 1}\right) \\
& =\left(\sqrt{2 J_{j}} \cos \left(\widetilde{\tilde{\omega}}_{j} t+\alpha_{j}\right), \sqrt{2 J_{j}} \sin \left(\widetilde{\widetilde{\omega}}_{j} t+\alpha_{j}\right)\right)_{j \geq 1} .
\end{aligned}
$$

Due to the assumption that $c$ is real and due to (3.41) we conclude that $\widetilde{\widetilde{\mathcal{S}}}^{(1)}$ is locally bounded on $U_{1}$. Moreover each component $\left(\sqrt{2 J_{j}} \cos \left(\widetilde{\tilde{\omega}}_{j} t+\right.\right.$ $\left.\left.\alpha_{j}\right) ; \sqrt{2 J_{j}} \sin \left(\widetilde{\tilde{\omega}}_{j} t+\alpha_{j}\right)\right)$ is analytic on $U_{1}$. By a version of $[\mathbf{P T}]$, Appendix A, Theorem 3, we conclude that $\widetilde{\mathcal{S}}^{(1)}$ is analytic on $U_{1}$ and (iii) is proved. To prove (ii) first note that, in particular, $\Omega$ is locally Lipschitz continuous. Taking into account that $\operatorname{Iso}\left(q_{j}\right)=\left\{\tilde{q} \in L^{2}\left(S^{1}\right) ; \operatorname{spec}\left(-\frac{d^{2}}{d x^{2}}+\tilde{q}\right)=\right.$ $\left.\operatorname{spec}\left(-\frac{d^{2}}{d x^{2}}+q_{j}\right)\right\}$ are compact in $L^{2}\left(S^{1}\right)(j=1,2)$ one concludes that there exists $M_{1}>0$, depending on $q_{1}$ and $q_{2}$, such that for any $\tilde{q}_{j} \in \operatorname{Iso}\left(q_{j}\right)$

$$
\left\|\Omega\left(\tilde{q}_{1}-c\right)-\Omega\left(\tilde{q}_{2}-c\right)\right\|_{\ell_{1 / 2}^{2}\left(\mathbb{R}^{2}\right)} \leq M_{1}\left\|\tilde{q}_{1}-\tilde{q}_{2}\right\|_{L^{2}\left(S^{1}\right)}
$$

where $c=\left[q_{1}\right]=\left[q_{2}\right]$. Using $\Omega^{-1}$ instead of $\Omega$, one concludes that there exists $M_{2}>0$ such that

$$
\left\|\tilde{q}_{1}-\tilde{q}_{2}\right\|_{L^{2}\left(S^{1}\right)} \leq M_{2}\left\|\Omega\left(\tilde{q}_{1}-c\right)-\Omega\left(\tilde{q}_{2}-c\right)\right\|_{\ell_{1 / 2}^{2}\left(\mathbb{R}^{2}\right)} .
$$


Therefore it suffices to show that, for any $t$,

$$
\begin{aligned}
& \left\|\Omega\left(\mathcal{S}^{(1)}\left(q_{1}\right)(\cdot, t)-c\right)-\Omega\left(\mathcal{S}^{(1)}\left(q_{2}\right)(\cdot, t)-c\right)\right\|_{\ell_{1 / 2}^{2}} \\
& \leq M\left\|q_{1}-q_{2}\right\|_{L^{2}}(1+|t|) .
\end{aligned}
$$

To simplify notation, write for $k=1,2$

$$
\left(x^{(k)}(t), y^{(k)}(t)\right)=\Omega\left(\mathcal{S}^{(1)}\left(q_{k}\right)(\cdot, t)-c\right) ; \omega_{j}^{(k)}=\omega_{j}\left(q_{k}\right) .
$$

Recall that for $k=1,2$ and $j \geq 1$

$$
\frac{d}{d t}\left(\begin{array}{l}
x_{j}^{(k)}(t) \\
y_{j}^{(k)}(t)
\end{array}\right)=\omega_{j}^{(k)}\left(\begin{array}{c}
y_{j}^{(k)}(t) \\
-x_{j}^{(k)}(t)
\end{array}\right)
$$

Therefore, with $\widetilde{\omega}_{j}^{(j)}:=\omega_{j}^{(k)}-(2 \pi j)^{3}-12[c] \pi j$,

$$
\begin{aligned}
& \frac{d}{d t}\left(\begin{array}{c}
x_{j}^{(1)}(t)-x_{j}^{(2)}(t) \\
y_{j}^{(1)}(t)-y_{j}^{(2)}(t)
\end{array}\right)=\tilde{\omega}_{j}^{(1)}\left(\begin{array}{c}
y_{j}^{(1)}(t) \\
-x_{j}^{(1)}(t)
\end{array}\right)-\widetilde{\omega}_{j}^{(2)}\left(\begin{array}{c}
y_{j}^{(2)}(t) \\
-x_{j}^{(2)}(t)
\end{array}\right) \\
& +\left((2 \pi j)^{3}+12[c] \pi j\right)\left(\begin{array}{cc}
y_{j}^{(1)}(t) & -y_{j}^{(2)}(t) \\
-\left(x_{j}^{(1)}(t)-x_{j}^{(2)}(t)\right)
\end{array}\right) .
\end{aligned}
$$

Scalar multiply (3.44) by $\left(\begin{array}{l}x_{j}^{(1)}(t)-x_{j}^{(2)}(t) \\ y_{j}^{(1)}(t)-y_{j}^{(2)}(t)\end{array}\right)$ to conclude that

$$
\begin{aligned}
& \frac{1}{2} \frac{d}{d t}\left|\begin{array}{c}
x_{j}^{(1)}(t)-x_{j}^{(2)}(t) \\
y_{j}^{(1)}(t)-y_{j}^{(2)}(t)
\end{array}\right|^{2} \\
& \leq\left|\tilde{\omega}_{j}^{(1)}\left(\begin{array}{c}
y_{j}^{(1)}(t) \\
-x_{j}^{(1)}(t)
\end{array}\right)-\tilde{\omega}_{j}^{(2)}\left(\begin{array}{c}
y_{j}^{(2)}(t) \\
-x_{j}^{(2)}(t)
\end{array}\right)\right|\left|\begin{array}{c}
x_{j}^{(1)}(t)-x_{j}^{(2)}(t) \\
y_{j}^{(1)}(t)-y_{j}^{(2)}(t)
\end{array}\right|
\end{aligned}
$$

Notice that $\left(\tilde{\omega}_{j}\left(y_{j}(t),-x_{j}(t)\right)\right)_{j \geq 1}$ is analytic on $U$ with values in $\ell_{1 / 2}^{2}\left(\mathbb{C}^{2}\right)$ due to the fact that $\tilde{\omega}_{j}=0(1)$ uniformly on $U$ (cf. Theorem 1$)$. Therefore

$$
\left\|\left(\tilde{\omega}_{j}^{(1)}\left(\begin{array}{c}
y_{j}^{(1)}(t) \\
-x_{j}^{(1)}(t)
\end{array}\right)-\tilde{\omega}_{j}^{(2)}\left(\begin{array}{c}
y_{j}^{(2)}(t) \\
-x_{j}^{(2)}(t)
\end{array}\right)\right)_{j \geq 1}\right\|_{\ell_{1 / 2}^{2}\left(\mathbb{C}^{2}\right)} \leq M\left\|q_{1}-q_{2}\right\|_{L^{2}}
$$

where $M>0$ is uniform for $t \in \mathbb{R}$. (Use compactness of the isospectral sets.) 
Combining (3.45) and (3.46) we conclude that

$$
\frac{d}{d t}\left\|\left(x^{(1)}(t), y^{(1)}(t)\right)-\left(x^{(2)}(t), y^{(2)}(t)\right)\right\|_{\ell_{1 / 2}^{2}} \leq M\left\|q_{1}-q_{2}\right\|_{L^{2}} .
$$

This proves estimate (3.43).

Using again properties of the map $\Omega$ one obtains in a straight forward way a result due to McKean-Trubowitz [MT1], and in its more general version, due to Bourgain [Bo1], concerning the almost periodicity of weak solutions of $K d V$. Recall (cf. [MT1], [Bo1]) that a function $u \in C\left(\mathbb{R} ; L^{2}\left(S^{1}\right)\right.$ ) is called almost periodic if for any $\epsilon>0$ there exists $0<T=T(\epsilon)<\infty$ so that, given any interval $I$ of length at least $T$, there exists $\tau_{0} \in I$ with the property

$$
\left\|u(\cdot, t)-u\left(\cdot, t+\tau_{0}\right)\right\|_{L^{2}}<\epsilon \quad \forall t \in \mathbb{R} .
$$

Proposition 3.7. Let $q \in L^{2}\left(S^{1}\right)$. The weak solution $\mathcal{S}^{(1)}(q)(\cdot, t)$ of $K d V$ is almost periodic.

Proof. For ease of exposition we assume that $[q]=0$ and write $v(\cdot, t)=$ $\mathcal{S}^{(1)}(q)(\cdot, t)$. Note that $v(\cdot, t) \in \operatorname{Iso}(q)$ and Iso $(q)$ is compact. Therefore $\Omega(\operatorname{Iso}(q))$ is compact as well and $\Omega^{-1}$ is uniformly continuous on $\Omega(\operatorname{Iso}(q))$. In particular, given $\epsilon>0$, there exists $\delta=\delta(\epsilon)>0$ such that for $z^{(1)}=$ $\left(x^{(1)}, y^{(1)}\right), z^{(2)}=\left(x^{(2)}, y^{(2)}\right)$ in $\Omega(\operatorname{Iso}(q))$ with $\left\|z^{(1)}-z^{(2)}\right\|_{\ell_{1 / 2}^{2}}<\delta$ one has $\left\|\Omega^{-1}\left(z^{(1)}\right)-\Omega^{-1}\left(z^{(2)}\right)\right\|_{L_{0}^{2}}<\epsilon$. It therefore suffices to prove that, given any $\delta>0$, there exists $0<T_{\delta}<\infty$ so that given any interval $I$ of length at least $T_{\delta}$, there exists $\tau_{0} \in I$ with the property that, for any $t \in R$,

$$
\left\|(x(t), y(t))-\left(x\left(t+\tau_{0}\right), y\left(t+\tau_{0}\right),\right)\right\|_{\ell_{1 / 2}^{2}}<\delta .
$$

To prove (3.47) we first note that there exists $N \geq 1$ such that, for any $t \in \mathbb{R}$

$$
\sum_{j=N+1}^{\infty} j\left(x_{j}(t)^{2}+y_{j}(t)^{2}\right)<(\delta / 4)^{2}
$$

where $\left(x_{j}(t), y_{j}(t)\right)$ is given by (3.34). Note that $\left(x_{j}(t), y_{j}(t)\right)_{1<j<N}$ is a quasi-periodic function of $t$. Therefore, given $\delta>0$, there exists $0<j<T_{\delta}<\infty$ so that in any interval $I$ of length at least $T_{\delta}$ one can find $\tau_{0} \in I$ with the property that, for any $t \in \mathbb{R}$,

$$
\sum_{j=1}^{N} j\left\{\left(x_{j}(t)-x_{j}\left(t+\tau_{0}\right)\right)^{2}+\left(y_{j}(t)-y_{j}\left(t+\tau_{0}\right)\right)^{2}\right\}<\left(\frac{\delta}{2}\right)^{2}
$$

and therefore (3.47) follows. 


\section{Frequencies of $K d V_{2}$.}

In this section we investigate the frequencies of the second Hamiltonian in the KdV hierarchy and apply the results to the initial value problem of $K d V_{2}$, (cf. e.g. [MM])

$$
\begin{gathered}
\partial_{t} v=\frac{1}{4} \partial_{x}^{5} v-\frac{5}{2} v \partial_{x}^{3} v-5 \partial_{x} v \partial_{x}^{2} v+\frac{15}{2} v^{2} \partial_{x} v \\
v(x ; 0)=q(x) .
\end{gathered}
$$

We want to construct weak solutions of (4.1)-(4.2) in $C\left(\mathbb{R} ; H^{1}\left(S^{1}\right)\right)$ for initial data $q$ in $H^{1}\left(S^{1}\right)$. The construction of the solution map $\mathcal{S}^{(2)}$ is very similar as in the case of $K d V$. Recall that the Hamiltonian $\mathcal{H}^{(2)}$ corresponding to $K d V_{2}$ is given by (cf. e.g. [MM])

$$
\mathcal{H}^{(2)}(q)=\int_{S^{1}}\left(\frac{1}{8}\left(\partial_{x}^{2} q\right)^{2}+\frac{5}{4} q\left(\partial_{x} q\right)^{2}+\frac{5}{8} q^{4}\right) d x .
$$

We point out that $\mathcal{H}^{(2)}$ is only defined for $q$ in $H^{2}\left(S^{1}\right)$. Nevertheless we will show that one can define the frequencies $\frac{\partial \mathcal{H}^{(2)}}{\partial J_{j}}$ corresponding to $\mathcal{H}^{(2)}$.

First notice that, with $p:=q-[q]$,

$$
\begin{aligned}
\mathcal{H}^{(2)}(q) & =\mathcal{H}^{(2)}(p+[q]) \\
& =\mathcal{H}^{(2)}(p)+\frac{5}{2}[q] \cdot \mathcal{H}^{(1)}(p)+\frac{15}{2}[q]^{2} \frac{1}{2} \int_{S^{1}} p^{2} d x+\frac{5}{8}[q]^{4} .
\end{aligned}
$$

Therefore it suffices to define the frequencies for $q \in H_{0}^{1}\left(S^{1}\right)$. Recall that for the construction of the $L_{2}$-integrable, holomorphic 1-forms $\Omega_{j}$ on $y=$ $\sqrt{\Delta^{2}-4}$ we have introduced the functions $f_{j}(\lambda):=C_{j} \frac{\varphi_{j}(\lambda)}{\sqrt{\Delta(\lambda)^{2}-4}}$ with

$$
\phi_{j}(\lambda):=\frac{1}{j^{2} \pi^{2}} \prod_{k \neq j} \frac{\mu_{k}^{(j)}-\lambda}{j^{2} \pi^{2}} ; C_{j}:=\left(\int_{\Gamma_{j}} \frac{\phi_{j}(u) d \mu}{\sqrt{\Delta(\mu)^{2}-4}}\right)^{-1}
$$

where $\Gamma_{j}$ is the counterclock oriented circle with center $\tau_{j}$ and sufficiently small radius $\rho_{j}>\gamma_{j} / 2$ and $C_{j}$ is a normalizing constant. The frequencies $\omega_{j}^{(2)}$ are then defined by

$$
\omega_{j}^{(2)}:=\frac{\pi i}{3} f_{j}^{(\mathrm{iv})}(\infty)
$$

where $f_{j}^{(\mathrm{iv})}(\infty)$ denotes the fourth deriviative of $f_{j}(\lambda)$ evaluated at infinity with respect to local coordinates $\tau^{2}=\frac{1}{\lambda}$ near $\lambda=\infty$. A straightforward 
calculation shows that

$$
\begin{aligned}
i \cdot & C_{j}^{-1} \frac{1}{4 !} f_{j}^{(\mathrm{iv})}(\infty)=\tau_{j}^{2}+\tau_{j}\left(\frac{\lambda_{0}}{2}-\sum_{k \neq j} \xi_{k}^{(j)}\right)+\frac{3}{2}\left(\frac{\lambda_{0}}{2}\right)^{2} \\
& -\frac{\lambda_{0}}{2} \sum_{k \neq j} \xi_{k}^{(j)}+\frac{1}{8} \sum_{k} \gamma_{k}^{2}+\frac{1}{2}\left(\sum_{k, l \neq j, k \neq l} \xi_{k}^{(j)} \xi_{l}^{(j)}\right)-\sum_{k \neq j} \tau_{k} \xi_{k}^{(j)}
\end{aligned}
$$

where $\xi_{k}^{(j)}:=\mu_{k}^{(j)}-\tau_{k}$ (as in Section 2). In view of Theorem 2.1, given $q_{0} \in H_{0}^{1}\left(S^{1}\right)$, there exists a neighborhood $U_{q_{0}}$ of $q_{0}$ in $H_{0}^{1}\left(S^{1} ; \mathbb{C}\right)$ so that the right hand side of (4.7) is well defined and analytic for any $j \geq 1$. In order to derive an asymptotic expansion of the $\omega_{j}^{(2)}$ 's we need a number of auxilary results.

From Theorem 2.7 we deduce the following

Lemma 4.1. Let $q_{0} \in L_{0}^{2}\left(S^{1}\right)$. Then there exist a neighborhood $U_{q_{0}}$ of $q_{0}$ in $L_{0}^{2}\left(S^{1} ; \mathbb{C}\right), j_{0} \geq 1$ and $C>0$ such that for $q \in U_{q_{0}}, j \geq j_{0}, k \geq j / 2$

$$
\left|\xi_{k}^{(j)}-\left(-\frac{1}{2} \frac{\left(\gamma_{k} / 2\right)^{2}}{\tau_{k}-\tau_{j}}-\frac{\left(\gamma_{k} / 2\right)^{2}}{4} \frac{1}{\tau_{k}-\lambda_{0}}\right)\right| \leq \frac{C}{j^{5}}\left|k \gamma_{k}\right|^{2}
$$

and, for $j \geq j_{0}, k \leq j / 2$

$$
\left|\xi_{k}^{(j)}-\left(-\frac{\left(\gamma_{k} / 2\right)^{2}}{4} \frac{1}{\tau_{k}-\lambda_{0}}\right)\right| \leq C \frac{\left|k \gamma_{k}\right|^{2}}{k^{5}} .
$$

Lemma 4.2. Let $q_{0} \in H_{0}^{1}\left(S^{1}\right)$. Then there exists a neighborhood $U_{q_{0}}$ of $q_{0}$ in $H_{0}^{1}\left(S^{1} ; \mathbb{C}\right)$ so that

$$
\left(\int_{\Gamma_{j}} \frac{\varphi_{j}(\mu) d \mu}{\sqrt{\Delta(\mu)^{2}-4}}\right)^{-1}=\left(2 \pi \psi_{j}(0)\right)^{-1}+O\left(\frac{\left|j \gamma_{j}\right|^{2}}{j^{5}}\right)
$$

$$
\begin{aligned}
\left(2 \psi_{j}(0)\right)^{-1}= & \left(\tau_{j}-\lambda_{0}\right)^{1 / 2}\left\{1-\sum_{k \leq j / 2} \frac{1}{\tau_{k}-\tau_{j}}\left(\xi_{k}^{(j)}+\frac{1}{2} \frac{\left(\gamma_{k} / 2\right)^{2}}{\tau_{k}-\tau_{j}}\right)\right. \\
& \left.+\frac{1}{2}\left(\sum_{k \leq j / 2} \frac{\xi_{k}^{(j)}}{\tau_{k}-\tau_{j}}\right)^{2}+\frac{1}{2} \sum_{k \leq j / 2}\left(\frac{\xi_{k}^{(j)}}{\tau_{k}-\tau_{j}}\right)^{2}\right\} \\
& +O\left(\frac{1}{j^{4}}\right) \sum_{k \geq j / 2}\left|k \gamma_{k}\right|^{2}+O\left(\frac{1}{j^{5}}\right) .
\end{aligned}
$$


Proof. (i) Follows from (3.20).

(ii) By Lemma 3.2,

$$
\begin{aligned}
\left(2 \psi_{j}(0)\right)^{-1}= & \left(\tau_{j}-\lambda_{0}\right)^{1 / 2}\left\{1-\sum_{k \neq j} \frac{1}{\tau_{k}-\tau_{j}}\left(\xi_{k}^{(j)}+\frac{1}{2} \frac{\left(\gamma_{k} / 2\right)^{2}}{\tau_{k}-\tau_{j}}\right)\right. \\
& \left.+\frac{1}{2}\left(\sum_{k \neq j} \frac{\xi_{k}^{(j)}}{\left(\tau_{k}-\tau_{j}\right)}\right)^{2}+\frac{1}{2} \sum_{k \neq j}\left(\frac{\xi_{k}^{(j)}}{\left(\tau_{k}-\tau_{j}\right)}\right)^{2}\right\}+O\left(\frac{1}{j^{5}}\right) .
\end{aligned}
$$

By (4.8i) one has

$$
\left|\sum_{k \geq j / 2} \frac{1}{\tau_{k}-\tau_{j}}\left(\xi_{k}^{(j)}+\frac{1}{2} \frac{\left(\gamma_{k} / 2\right)^{2}}{\tau_{k}-\tau_{j}}\right)\right| \leq O\left(\frac{1}{j^{5}}\right) \sum_{k \geq j / 2}\left|\gamma_{k} k\right|^{2} .
$$

Similarly, by (4.8i) and (4.8ii),

$$
\sum_{k \geq j / 2} \frac{\xi_{k}^{(j)}}{\tau_{k}-\tau_{j}}=O\left(\frac{1}{j^{4}}\right) ; \quad \sum_{k \leq j / 2} \frac{\xi_{k}^{(j)}}{\tau_{k}-\tau_{j}}=O\left(\frac{1}{j^{2}}\right) .
$$

This leads to (4.9ii).

Lemma 4.3. Let $q_{0} \in H_{0}^{1}\left(S^{1}\right)$. Then there exists a neighborhood $U_{q_{0}}$ of $q_{0}$ in $H_{0}^{1}\left(S^{1} ; \mathbb{C}\right)$ and $C>0$ so that for any $q$ in $U_{q_{0}}$ and $j \geq 1$

$$
\left|\omega_{j}^{(2)}-8\left((j \pi)^{5}+j \pi 5 \tilde{\tau}_{j}\right)\right| \leq C\left(\sum_{k \geq j / 2}\left|k \gamma_{k}\right|^{2}+\frac{1}{j}\right)
$$

where $\sqrt{\tau_{j}}=j \pi+\frac{\tilde{\tau}_{j}}{(j \pi)^{3}}$.

Proof. Using (4.7), (4.6) and (4.5) one obtains

$$
\begin{aligned}
\omega_{j}^{(2)}= & 8 \pi\left(\int_{\Gamma_{j}} \frac{\varphi_{j}(\mu) d \mu}{\sqrt{\Delta(\mu)^{2}-4}}\right)^{-1}\left\{\tau_{j}^{2}+\tau_{j}\left(\frac{\lambda_{0}}{2}-\sum_{k \neq j} \xi_{k}^{(j)}\right)\right. \\
& \left.+\frac{3}{8} \lambda_{0}^{2}-\frac{\lambda_{0}}{2} \sum_{k \neq j} \xi_{k}^{(j)}+\frac{1}{8} \sum_{k \neq j} \gamma_{k}^{2}+\frac{1}{2} \sum_{k, l \neq j, k \neq l} \xi_{k}^{(j)} \xi_{l}^{(j)}-\sum_{k \neq j} \tau_{k} \xi_{k}^{(j)}\right\} .
\end{aligned}
$$

Therefore with $\left(\int_{\Gamma_{j}} \frac{\varphi_{j}(\mu) d \mu}{\sqrt{\Delta(\mu)^{2}-4}}\right)^{-1}=\left(2 \pi \psi_{j}(0)\right)^{-1}+O\left(\frac{\left|j \gamma_{j}\right|^{2}}{j^{5}}\right)$ and the asymptotics of $\left(2 \pi \psi_{j}(0)\right)^{-1}$ we obtain by a straight forward computation

$$
\frac{1}{8} \omega_{j}^{(2)}=\tau_{j}^{5 / 2}+O(1)\left(\sum_{k \geq j / 2}\left|k \gamma_{k}\right|^{2}\right)+O\left(\frac{1}{j}\right) .
$$


With $\sqrt{\tau_{j}}=j \pi+\frac{\tilde{\tau}_{j}}{(j \pi)^{3}}$ we obtain

$$
\tau_{j}^{5 / 2}=(j \pi)^{5}+\left(5 \tilde{\tau}_{j}\right) j \pi+O\left(\frac{1}{j}\right)
$$

which leads to (4.10).

Lemma 4.4. Let $q_{0} \in H_{0}^{1}\left(S^{1}\right)$. Then there exists a neighborhood $U_{q_{0}}$ of $q_{0}$ in $H_{0}^{1}\left(S^{1} ; \mathbb{C}\right)$ and $C>0$ so that, uniformly for $q$ in $U_{q_{0}}$,

$$
\begin{aligned}
\tau_{j}(q) & =j^{2} \pi^{2}+\frac{1}{4} \frac{\int_{0}^{1} q(x)^{2} d x}{j^{2} \pi^{2}}+O\left(\frac{1}{j^{3}}\right) \\
\sqrt{\tau_{j}(q)} & =j \pi+\frac{1}{8} \frac{\int_{0}^{1} q(x)^{2} d x}{(j \pi)^{3}}+O\left(\frac{1}{j^{4}}\right) .
\end{aligned}
$$

Proof. Notice that (4.11ii) follows immediately from (4.11i), so let us concentrate on (4.11i). Recall that, for $j \geq j_{0}$ with $j_{0}$ sufficiently large,

$$
2 \tau_{j}=\operatorname{tr}\left(\left(-\frac{d^{2}}{d x^{2}}+q\right) P_{j}\right)
$$

where $P_{j}$ is the Riesz projector on the subspace generated by the generalized eigenfunctions corresponding to the eigenvalues $\lambda_{2 j}$ and $\lambda_{2 j-1}$. It is convenient to introduce, for $j \geq j_{0}$,

$$
\begin{aligned}
L(t) & :=-\frac{d^{2}}{d x^{2}}+t q, \\
P_{j}(t) & :=\frac{1}{2 \pi i} \int_{\Gamma_{j}}(\lambda-L(t))^{-1} d \lambda, \\
\tau_{j}(t) & :=\operatorname{tr}\left(L(t) P_{j}(t)\right)
\end{aligned}
$$

where $\Gamma_{j}$ is a circle with center $j^{2} \pi^{2}$ and radius $\rho_{j}$ so that the eigenvalues $\lambda_{2 j}(t q)$ and $\lambda_{2 j-1}(t q)$ are inside of $\Gamma_{j}$ and all other eigenvalues are outside of $\Gamma_{j}$ for any $0 \leq t \leq 1, q \in U_{q_{0}}$. (The integer $j_{0}$ has been chosen sufficiently large and the neighborhood $U_{q_{0}}$ sufficiently small.) The functions $\tau_{j}(t)$ admit a Taylor expansion,

$$
\tau_{j}(1)=\tau_{j}(0)+\tau_{j}^{\prime}(0)+\tau_{j}^{\prime \prime}(0) \frac{1}{2 !}+\tau_{j}^{\prime \prime \prime}(0) \frac{1}{3 !}+\mathcal{E}_{j}
$$

where $\mathcal{E}_{j}$ denotes the Taylor remainder term. Clearly

$$
2 \tau_{j}(0)=n^{2} \pi^{2} \operatorname{tr} P_{j}(0)=2 j^{2} \pi^{2} .
$$


Further

$$
\begin{aligned}
2 \tau_{j}^{\prime}(0) & =\operatorname{tr}\left(P_{j}(0) L^{\prime}(0) P_{j}(0)\right)=\operatorname{tr}\left(q P_{j}(0)\right) \\
& =2[q]=0
\end{aligned}
$$

To compute the second derivative it is convenient to introduce

$$
\hat{q}_{k}=\frac{1}{2} \int_{-1}^{1} e^{-i \pi k x} q(x) d x .
$$

Then

$$
\begin{aligned}
2 \tau_{j}^{\prime \prime}(0) & =\operatorname{tr}\left(q P_{j}^{\prime}(0)\right)=\sum_{m, k} \hat{q}_{m-k} \hat{q}_{k-m} \frac{1}{2 \pi i} \int_{\Gamma_{j}} \frac{d \lambda}{\left(\lambda-m^{2} \pi^{2}\right)\left(\lambda-k^{2} \pi^{2}\right)} \\
& =4 \sum_{m \neq \pm j} \frac{\hat{q}_{m-j} \hat{q}_{j-m}}{\pi^{2}\left(j^{2}-m^{2}\right)}=\frac{4}{\pi^{2}} \sum_{k \neq 0,2 j} \frac{\hat{q}_{n} \hat{q}_{-n}}{n(2 j-n)} .
\end{aligned}
$$

Writing

$$
\begin{aligned}
\frac{1}{n(2 j-n)} & =\frac{1}{n} \frac{1}{2 j}+\frac{1}{2 j-n} \frac{1}{2 j} \\
& =\frac{1}{n} \frac{1}{2 j}+\frac{1}{(2 j)^{2}}+\frac{n}{(2 j-n)(2 j)^{2}}
\end{aligned}
$$

and using that

$$
\sum_{n \neq 0,2 j} \frac{\hat{q}_{n} \hat{q}_{-n}}{n}=\sum_{n \neq 0} \frac{\hat{q}_{n} \hat{q}_{-n}}{n}-\frac{\hat{q}_{2 j} \hat{q}_{-2 j}}{2 j}=0-\frac{\hat{q}_{2 j} \hat{q}_{-2 j}}{2 j}
$$

we conclude from (4.14)

$$
\begin{aligned}
& 2 \tau_{j}^{\prime \prime}(0)=\frac{4}{\pi^{2}} \sum_{n \neq 0} \frac{\hat{q}_{n}}{\hat{q}_{-n}}(2 j)^{2}+\frac{4}{\pi^{2}} \sum_{\substack{n \neq 0 \\
n \neq 2 j}} \frac{n \hat{q}_{n}(-n) \hat{q}_{-n}}{(-n)(2 j-n)(2 j)^{2}} \\
& =\frac{1}{\pi^{2} j^{2}} \frac{1}{2} \int_{-1}^{1} q(x)^{2} d x+0\left(\frac{1}{j^{3}}\right)=\frac{1}{j^{2} \pi^{2}} \int_{0}^{1} q(x)^{2} d x+O\left(\frac{1}{j^{3}}\right) .
\end{aligned}
$$

Similarly, we compute $\tau_{j}^{\prime \prime \prime}(0)$,

$$
2 \tau_{j}^{\prime \prime \prime}(0)=\operatorname{tr}\left(q P_{j}^{\prime \prime}(0)\right)
$$




$$
\begin{aligned}
& =\operatorname{tr} \frac{1}{2 \pi i} \int_{\Gamma_{j}} q 2(\lambda-L(0))^{-1} q(\lambda-L(0))^{-1} q(\lambda-L(0))^{-1} d \lambda \\
& =2 \sum_{m, k, n} \hat{q}_{m-n} \hat{q}_{n-k} \hat{q}_{k-m} \frac{1}{2 \pi i} \int_{\Gamma_{j}} \frac{d \lambda}{\left(\lambda-n^{2} \pi^{2}\right)\left(\lambda-k^{2} \pi^{2}\right)\left(\lambda-m^{2} \pi^{2}\right)} \\
& =6 \cdot 2 \sum_{\substack{n \neq \pm j \\
k \neq \pm j}} \frac{\hat{q}_{j-n} \hat{q}_{n-k} \hat{q}_{k-j}}{\left(j^{2} \pi^{2}-n^{2} \pi^{2}\right)\left(j^{2} \pi^{2}-k^{2} \pi^{2}\right)} \\
& =\frac{6 \cdot 2}{\pi^{4}} \sum_{\substack{m, \ell \\
m, \ell \neq 0,2 j}} \frac{\hat{q}_{m} \hat{q}_{\ell-m} \hat{q}_{-\ell}}{m(2 j-m) \ell(2 j-\ell)} .
\end{aligned}
$$

Again, writing

$$
\begin{aligned}
\frac{1}{m(2 j-m)} \frac{1}{(2 j-\ell) \ell}= & \frac{1}{m \ell(2 j)^{2}}+\frac{m}{m(2 j-m) \ell(2 j)^{2}}+\frac{\ell}{m \ell(2 j-\ell)} \frac{1}{(2 j)^{2}} \\
& +\frac{m \ell}{\ell(2 j-\ell) m(2 j-m)} \frac{1}{(2 j)^{2}}
\end{aligned}
$$

and taking into account that $q \in U_{q_{0}} \subset H_{0}^{1}\left(S^{1} ; \mathbb{C}\right)$ we conclude that

$$
\begin{aligned}
2 \tau_{j}^{\prime \prime \prime}(0) & =\frac{6 \cdot 2}{\pi^{4}} \sum_{m, \ell \neq 0} \frac{\hat{q}_{m} \hat{q}_{\ell-m} \hat{q}_{-\ell}}{m \ell(2 j)^{2}}+O\left(\frac{1}{j^{3}}\right) \\
& =0+O\left(\frac{1}{j^{3}}\right) .
\end{aligned}
$$

Using the standard resolvent estimate one concludes that the Taylor remainder term $\mathcal{E}_{j}$ satisfies

$$
\mathcal{E}_{j}=O\left(\frac{1}{j^{3}}\right)
$$

Substitute (4.13), (4.14), (4.16), (4.18) and (4.19) into the Taylor expansion (4.12) we conclude

$$
\tau_{j}(1)=j^{2} \pi^{2}+\frac{\frac{1}{4} \int_{0}^{1} q(x)^{2} d x}{j^{2} \pi^{2}}+O\left(\frac{1}{j^{3}}\right) .
$$

This proves (4.11i).

Theorem 4.5. Let $q_{0} \in H_{0}^{1}\left(S^{1}\right)$. Then there exists a neighborhood $U_{q_{0}}$ of $q_{0}$ in $H_{0}^{1}\left(S^{1} ; \mathbb{C}\right)$ and $C>0$ so that uniformly for $q$ in $U_{q_{0}}$

$$
\left|\omega_{j}^{(2)}-8\left[(j \pi)^{5}-a_{1} j \pi\right]\right| \leq C
$$


where $a_{1}=a_{1}(q)$ is given by

$$
a_{1}=\frac{5}{8} \int_{0}^{1} q(x)^{2} d x
$$

Proof. Combine the estimate (4.10) for $\omega_{j}^{(2)}$ with the estimate (4.11ii) for $\tilde{\tau}_{j}$

$$
\tilde{\tau}_{j}=\frac{1}{8} \int_{0}^{1} q(x)^{2} d x+0\left(\frac{1}{j}\right)
$$

to obtain (4.20)-(4.21).

Given real numbers $c_{1}, c_{3}$ we now construct weak solutions of (4.1)-(4.2) in $C\left(\mathbb{R} ; H_{c_{1}, c_{3}}^{1}\left(S^{1}\right)\right)$ for initial data $q$ in $H_{c_{1}, c_{3}}^{1}\left(S^{1}\right):=\left\{q \in H^{1}\left(S^{1}\right) ;[q]=\right.$ $\left.c_{3} ; a_{1}(q)=c_{1}\right\}$.

Theorem 4.6. Let $c_{1}, c_{3} \in \mathbb{R}$. Then there exists a solution map $\mathcal{S}^{(2)}$

$$
\mathcal{S}^{(2)}: H_{c_{1}, c_{3}}^{1}\left(S^{1}\right) \rightarrow C\left(\mathbb{R}, H_{c_{1}, c_{3}}^{1}\left(S^{1}\right)\right)
$$

with the following properties:

(i) $\mathcal{S}^{(2)}(q)$ is a weak solution of (4.1)-(4.2);

(ii) given $q_{1}, q_{2}$ in $H^{1}\left(S^{1}\right)$ with

$$
\left[q_{1}\right]=\left[q_{2}\right]=c_{3} ; a_{1}\left(q_{1}\right)=a_{1}\left(q_{2}\right)=c_{1}
$$

there exists $M>0$ so that for any $t$

$$
\left\|\mathcal{S}^{(2)}\left(q_{1}\right)(\cdot, t)-\mathcal{S}^{(2)}\left(q_{2}\right)(\cdot, t)\right\|_{H^{1}\left(S^{1}\right)} \leq M(1+|t|)\left\|q_{1}-q_{2}\right\|_{H^{1}\left(S^{1}\right)} .
$$

(iii) For any $0<T<\infty, \mathcal{S}^{(2)}: H_{c_{1}, c_{3}}^{1}\left(S^{1}\right) \rightarrow C\left([-T, T] ; H_{c_{1}, c_{3}}^{1}\left(S^{1}\right)\right)$ is real analytic.

Remark 1. According to [Bo2], Bourgain's method cannot be applied to solve the initial value problem (4.1)-(4.2) in $H^{1}\left(S^{1}\right)$.

Remark 2. Note that the $K d V$ Hamiltonian $\mathcal{H}(q)=\int_{S^{1}}\left(\frac{1}{2}\left(\partial_{x} q\right)^{2}+q^{3}\right) d x$ is a conserved quantity for (4.1), as well as the average $[q]$ and $\int_{S^{1}} q(x)^{2} d x$. Thus, given a real valued smooth solution $v(x, t)$ of $(4.1)$ one obtains an a priori bound for $\int_{S^{1}}\left|\partial_{x} v(x, t)\right|^{2} d x$ which leads to the existence of a weak solution of (4.1)-(4.2). This solution can be approximated by finite gap solutions of (4.1). Of course, the main point of the statement in Theorem 4.6 is that the solution map $\mathcal{S}^{(2)}$ is real analytic.

Proof of Theorem 4.6. The construction of the solution map $\mathcal{S}^{(2)}$ and the proofs of its properties are similar to the ones of $\mathcal{S}^{(1)}$ and we therefore omit it. 


\section{Appendix: Proof of Theorem 2.1.}

In the case where the potential $q \in L_{0}^{2}\left(S^{1} ; \mathbb{C}\right)$ is real valued, the entire functions $\phi_{j}(\lambda, q)(j \geq 1)$ are constructed in [MT2]. Actually, instead of $\phi_{j}$, [MT2] construct entire functions $1_{j}(\lambda, q)$ which coincide with $\phi_{j}(\lambda, q)$ up to a normalization factor. (In the sequel, we use the normalization introduced by McKean-Trubowitz.) By a straightforward perturbation argument one shows that for given $q_{0} \in L_{0}\left(S^{1} ; \mathbb{R}\right)$ there exist a (sufficiently small) neighborhood $U_{q_{0}}$ of $q_{0}$ in $L_{0}^{2}\left(S^{1} ; \mathbb{C}\right)$ so that for $q \in U_{q_{0}}$ the functions $1_{j}(\lambda, q)(j \geq 1)$ are uniquely defined and analytic with respect to $q$. The main part of the proof of Theorem 2.1 consists in analyzing the zeroes of $1_{j}(\lambda, q)$ for $q$ in a neighborhood $U \subseteq U_{q_{0}}$ of $q_{0}$ in $L_{0}^{2}\left(S^{1} ; \mathbb{C}\right)$ which does not depend on $j$ and proving the estimates (2.1). We proceed in two steps:

In Section A.2 we show, using Rouché's theorem that, given $q_{0} \in L_{0}^{2}\left(S^{1} ; \mathbb{R}\right)$, $0<K \leq K\left(q_{0}\right)$ (cf. definition after (A.2)) and $N \in \mathbb{N}$ arbitrary, there exist a neighborhood $U_{q_{0}, K, N}$ of $q_{0}$ in $L_{0}^{2}\left(S^{1} ; \mathbb{C}\right.$ ) (depending on $q_{0}, K$ and $N$ ) so that, for any $j \geq 1, q \in U_{q_{0}, K, N}$ and $1 \leq k \leq N, k \neq j$, the entire function $1_{j}(\lambda, q)$ has precisely one zero, denoted by $\mu_{k}^{(j)}(q)$, inside the circle $\Gamma_{k}(K)$ (defined in Section A.1). We point out that $\bigcap_{N \geq 1} U_{q_{0}, K, N}$ might consist of $\left\{q_{0}\right\}$ only and therefore we need an additional argument for the proof of Theorem 2.1 .

In Section A.3, we show that there exist a neighborhood $U$ of $q_{0}$ in $L_{0}^{2}\left(S^{1} ; \mathbb{C}\right)$ and $N \geq 1$, depending on $U$ only, so that the system of nonlinear equations for the zeroes $\mu_{k}^{(j)}(q)$ of $1_{j}(\lambda, q)$ with $k \geq N+1, k \neq j$, obtained from (2.2), can be solved by a contraction argument. At the same time we obtain the estimates for $\mu_{k}^{(j)}(q)-\tau_{k}(q)$ claimed in Theorem 2.1. Finally the analyticity of the zeroes $\mu_{k}^{(j)}(q)$ of $1_{j}(\lambda)$ follows from Cauchy's integral formula. We remark that by using the contraction argument of section A.3 we can obtain a new proof for the existence of $1_{j}(\lambda, q)$ and their product representation for real valued potentials $\mathrm{q}$ in $L_{0}^{2}$.

In Section A.4 we present the proof of Theorem 2.1, combining the results of Sections A.1, A.2 and A.3.

In order to avoid introducing cumbersome notation the same letter $\mathrm{C}$ will denote various constants and the same letter $\mathrm{U}$ will denote various neighborhoods.

\section{A.1. Normalized Riesz basis of holomorphic differentials.}

Let us first recall some notions, notations and results from [MT1] (cf. also [MT2]). Denote by $I_{3 / 2}$ the complex Hilbert space of entire functions 
$\phi$ of order $\leq 1 / 2$ and type $\leq 1$ with

$$
\|\phi\|_{I_{3 / 2}}^{2}:=\int_{0}^{\infty}|\phi(\lambda)|^{2} \lambda^{3 / 2} d \lambda<\infty .
$$

The inner product in $I_{3 / 2}$ is given by

$$
\langle\phi, \psi\rangle:=\int_{0}^{\infty} \phi(\lambda) \overline{\psi(\lambda)} \lambda^{3 / 2} d \lambda
$$

By the Paley-Wiener theorem

$$
|\lambda \phi(\lambda)| \leq\|\phi\|_{I_{3 / 2}} e^{\mid \operatorname{Im} \sqrt{\lambda \mid}} .
$$

Introduce, for $q_{0} \in L_{0}^{2}\left(S^{1} ; \mathbb{R}\right), K\left(q_{0}\right):=\frac{1}{5} \min _{n \geq 0}\left(\lambda_{2 n+1}\left(q_{0}\right)-\lambda_{2 n}\left(q_{0}\right)\right)$ where $\left(\lambda_{n}\left(q_{0}\right)\right)_{n>0}$ is the anti/periodic spectrum of $-\frac{d^{2}}{d x^{2}}+q_{0}$. Then $K\left(q_{0}\right)>0$ and for $0<K \leq K\left(q_{0}\right)$ there exist a bounded neighborhood $V \equiv V_{q_{0}, K}$ of $q_{0}$ in $L_{0}^{2}\left(S^{1} ; \mathbb{C}\right)$ so that for $q \in V$, the anti/periodic eigenvalues $\left(\lambda_{n}(q)\right)_{n \geq 0}$ satisfy

$$
\sup _{j \geq 0}\left|\lambda_{j}(q)-\lambda_{j}\left(q_{0}\right)\right| \leq K
$$

Denote by $\Gamma_{n}=\Lambda_{n}\left(K, q_{0}\right)$ the counterclockwise oriented circle in $\mathbb{C}$ with center $\tau_{n}=\tau_{n}\left(q_{0}\right)=\frac{\lambda_{2 n}\left(q_{0}\right)+\lambda_{2 n-1}\left(q_{0}\right)}{2}$ and radius $r_{n}:=\frac{\gamma_{n}\left(q_{0}\right)}{2}+2 K$ where $\gamma_{n}\left(q_{0}\right)=\lambda_{2 n}\left(q_{0}\right)-\lambda_{2 n-1}\left(q_{0}\right)$. Notice that, because of (A.3), for $q$ in $V$, $\lambda_{2 n}(q)$ and $\lambda_{2 n-1}(q)$ are inside $\Gamma_{n}$ and all other eigenvalues $\lambda_{k}(q)$ are outside $\Gamma_{n}$.

For $q$ in $V$ and $n \geq 1$, denote by $A_{n}(q): I_{3 / 2} \rightarrow \mathbb{C}$ the bounded linear functional

$$
A_{n}(q)(\phi):=\int_{\Gamma_{n}} \frac{\phi(\lambda) d \lambda}{\sqrt{\Delta(\lambda, q)^{2}-4}} .
$$

Then $A_{n}(q)$ is an analytic function of $q \in V$ with values in the dual $I_{3 / 2}^{*}$ of $I_{3 / 2}$. Using (A.2) and (2.6) one verifies (cf.[MT 1, 2]) that there exists $C \geq 1$ such that $\frac{1}{C} \leq\left\|n A_{n}(q)\right\|_{I_{3 / 2}^{*}} \leq C$ for $q$ in $V$.

Recall (cf. [GK, p. 310]) that $\left(u_{n}\right)_{n \geq 1}$ is said to be a Riesz basis of a Hilbert space $H$ if there exist an orthonormal basis $\left(e_{j}\right)_{j \geq 1}$ of $H$ and an invertible bounded linear operator $B: H \rightarrow H$ such that $B e_{j}=u_{j}(j \geq$ 1 ). In the case where the potential $q$ is real valued McKean-Trubowitz (cf. [MT2]) showed that $\left(n A_{n}(q)\right)_{n \geq 1}$ is a Riesz basis of $I_{3 / 2}^{*}$. To be able to consider complex valued potentials as well, we need the following auxilary result. We recall (use Marchenko's asymptotics of the eigenvalues $\lambda_{j}(q)$ ) (cf 
[Ma, Theorem 1.5.2]) that the map $q \mapsto\left(\tau_{k}(q)-k^{2} \pi^{2}\right)_{k \geq 1}$ is an analytic function on $V$ with values in $\ell_{1}^{2}(\mathbb{N} ; \mathbb{C})$.

Lemma A.1. There exist $C \geq 1$ and $N \geq 1$ such that for $n \geq N$ and $q \in V(V$ defined before (A.3))

$$
\begin{aligned}
& n\left\|A_{n}(q)-A_{n}\left(q_{0}\right)\right\|_{I_{3 / 2}^{*}} \leq C\left(\left|\gamma_{n}(q)^{2}-\gamma_{n}\left(q_{0}\right)^{2}\right|\right) \\
& +\frac{C}{n^{2}}\left(\left|\lambda_{0}(q)-\lambda_{0}\left(q_{0}\right)\right|+\left(\sum_{k \geq 1} k^{2}\left|\tau_{k}(q)-\tau_{k}\left(q_{0}\right)\right|^{2}\right)^{1 / 2}\right. \\
& \left.\quad+\left(\sum_{k \geq 1}\left|\gamma_{k}(q)^{2}-\gamma_{k}\left(q_{0}\right)^{2}\right|\right)^{1 / 2}\right) .
\end{aligned}
$$

Proof. For $\phi \in I_{3 / 2}, n \geq 1$ and $q \in V$

$$
\begin{aligned}
& A_{n}(q)(\phi)-A_{n}\left(q_{0}\right)(\phi) \\
& =\int_{\Gamma_{n}} \frac{\left(\left(\Delta\left(\lambda, q_{0}\right)^{2}-4\right)-\left(\Delta(\lambda, q)^{2}-4\right)\right) \phi(\lambda) d \lambda}{\sqrt{\Delta(\lambda, q)^{2}-4} \sqrt{\Delta\left(\lambda, q_{0}\right)^{2}-4}\left(\sqrt{\Delta(\lambda, q)^{2}-4}+\sqrt{\Delta\left(\lambda, q_{0}\right)^{2}-4}\right)} .
\end{aligned}
$$

Recall that for $\lambda \in \Gamma_{n}$ (cf. e.g. [Ka, p. 547])

$$
\Delta(\lambda, q)^{2}-4=\frac{1}{n^{2} \pi^{2}}\left(\lambda_{2 n}(q)-\lambda\right)\left(\lambda-\lambda_{2 n-1}(q)\right)\left(1+\beta_{n}(\lambda, q) \frac{\log (n+1)}{n}\right)
$$

where $\beta_{n}(\lambda, q)$ satisfies $\left|\beta_{n}(\lambda, q)\right|<C$ for $n \geq 1, q \in V$ for some $C>0$.

In view of the choice of $K, \Gamma_{n}=\Gamma_{n}(K)$ and $V$, these asymptotics can be used to obtain an estimate of the denominator of the integrand in (A.5), for $q$ in $V$,

$$
\begin{aligned}
& \sup _{\lambda \in \Gamma_{n}}\left|\frac{1}{\sqrt{\Delta(\lambda, q)^{2}-4}} \cdot \frac{1}{\sqrt{\Delta\left(\lambda, q_{0}\right)^{2}-4}} \cdot \frac{1}{\sqrt{\Delta(\lambda, q)^{2}-4}+\sqrt{\Delta\left(\lambda, q_{0}\right)^{2}-4}}\right| \\
& \leq C n^{3}
\end{aligned}
$$

where $C \geq 1$ can be chosen independently of $q \in V$ and $n \geq 1$.

To estimate the nominator of the integrand in (A.5) we write

$$
\left(\Delta\left(\lambda, q_{0}\right)^{2}-4\right)-\left(\Delta(\lambda, q)^{2}-4\right)=\left(\Delta\left(\lambda, q_{0}\right)^{2}-4\right)\left(1-\frac{\Delta(\lambda, q)^{2}-4}{\Delta\left(\lambda, q_{0}\right)^{2}-4}\right)
$$


Use the product representation

$$
\Delta(\lambda, q)^{2}-4=4\left(\lambda_{0}(q)-\lambda\right) \prod_{k=1}^{\infty} \frac{\left(\lambda_{2 k}(q)-\lambda\right)\left(\lambda_{2 k-1}(q)-\lambda\right)}{k^{4} \pi^{4}}
$$

and write $\left(\lambda_{2 k}(q)-\lambda\right)\left(\lambda_{2 k-1}(q)-\lambda\right)=\left(\tau_{k}(q)-\lambda\right)^{2}-\left(\frac{\gamma_{k}(q)}{2}\right)^{2}$ to obtain

$$
\begin{aligned}
& \frac{\Delta(\lambda, q)^{2}-4}{\Delta\left(\lambda, q_{0}\right)^{2}-4}=\left(1+\frac{\lambda_{0}\left(q_{0}\right)-\lambda_{0}(q)}{\lambda-\lambda_{0}\left(q_{0}\right)}\right) \\
& \left(1+\frac{\left(\tau_{n}(q)-\tau_{n}\left(q_{0}\right)\right)\left(\tau_{n}(q)+\tau_{n}\left(q_{0}\right)-2 \lambda\right)-\left(\frac{\gamma_{n}(q)}{2}\right)^{2}+\left(\frac{\gamma_{n}\left(q_{0}\right)}{2}\right)^{2}}{\left(\tau_{n}\left(q_{0}\right)-\lambda\right)^{2}-\left(\frac{\gamma_{n}\left(q_{0}\right)}{2}\right)^{2}}\right) \cdot E_{n}
\end{aligned}
$$

where

$$
E_{n}:=\prod_{\substack{k \neq n \\ k \geq 1}}\left(1+\frac{\left(\tau_{k}(q)-\tau_{k}\left(q_{0}\right)\right)\left(\tau_{k}(q)+\tau_{k}\left(q_{0}\right)-2 \lambda\right)-\left(\frac{\gamma_{k}(q)}{2}\right)^{2}+\left(\frac{\gamma_{k}\left(q_{0}\right)}{2}\right)^{2}}{\left(\tau_{k}\left(q_{0}\right)-\lambda\right)^{2}-\left(\frac{\gamma_{k}\left(q_{0}\right)}{2}\right)^{2}}\right) .
$$

One verifies that there exist $N_{0} \geq 1$ and $C \geq 1$ such that for $n \geq N_{0}, q \in V$ and $\lambda \in \Gamma_{n}$

$$
\left|E_{n}-1\right| \leq C \sum_{\substack{k \neq n \\ k \geq 1}}\left(\frac{\left|\tau_{k}(q)-\tau_{k}\left(q_{0}\right)\right|\left|k^{2}-n^{2}\right|}{\left|k^{2}-n^{2}\right|^{2}}+\frac{\left|\left(\frac{\gamma_{k}(q)}{2}\right)^{2}-\left(\frac{\gamma_{k}\left(q_{0}\right)}{2}\right)^{2}\right|}{\left|k^{2}-n^{2}\right|^{2}}\right) .
$$

Notice that

$$
\sum_{\substack{k \neq n \\ k \geq 1}} \frac{\left|\left(\frac{\gamma_{k}(q)}{2}\right)^{2}-\left(\frac{\gamma_{k}\left(q_{0}\right)}{2}\right)^{2}\right|}{\left|k^{2}-n^{2}\right|^{2}} \leq \frac{1}{n^{2}} \sum_{k \geq 1}\left|\left(\frac{\gamma_{k}(q)}{2}\right)^{2}-\left(\frac{\gamma_{k}\left(q_{0}\right)}{2}\right)^{2}\right|
$$

and

$$
\sum_{\substack{|k-n| \geq \frac{n}{2} \\ k \geq 1}} \frac{\left|\tau_{k}(q)-\tau_{k}\left(q_{0}\right)\right|\left|k^{2}-n^{2}\right|}{\left|k^{2}-n^{2}\right|^{2}} \leq \frac{C}{n^{2}} \sum_{k \geq 1}\left|\tau_{k}(q)-\tau_{k}\left(q_{0}\right)\right| .
$$

Further

$$
\sum_{\substack{|k-n| \leq \frac{n}{2} \\ k \neq n}} \frac{\left|\tau_{k}(q)-\tau_{k}\left(q_{0}\right)\right|\left|k^{2}-n^{2}\right|}{\left|k^{2}-n^{2}\right|^{2}}
$$




$$
\begin{aligned}
& \leq \frac{C}{n^{2}} \sum_{\substack{|k-n| \leq \frac{n}{2} \\
k \neq n}} \frac{1}{|k-n|} k\left|\tau_{k}(q)-\tau_{k}\left(q_{0}\right)\right| \\
& \leq \frac{C}{n^{2}}\left(\sum_{|k-n| \geq 1} \frac{1}{|k-n|^{2}}\right)^{1 / 2}\left(\sum_{k \geq 1} k^{2}\left|\tau_{k}(q)-\tau_{k}\left(q_{0}\right)\right|^{2}\right)^{1 / 2} .
\end{aligned}
$$

Combining the above estimates we conclude that there exist $N \geq 1$ and $C \geq 1$ such that for $n \geq N, q \in V, \lambda \in \Gamma_{n}$

$$
\begin{aligned}
& \left(\mathrm{A} .6^{\prime \prime}\right) \\
& \left|1-\frac{\Delta(\lambda, q)^{2}-4}{\Delta\left(\lambda, q_{0}\right)^{2}-4}\right| \\
& \leq C\left|\tau_{n}(q)-\tau_{n}\left(q_{0}\right)\right|+C\left|\left(\frac{\gamma_{n}(q)}{2}\right)^{2}-\left(\frac{\gamma_{n}\left(q_{0}\right)}{2}\right)^{2}\right|+\frac{C}{n^{2}}\left|\lambda_{0}(q)-\lambda_{0}\left(q_{0}\right)\right| \\
& +\frac{C}{n^{2}}\left(\sum_{k \geq 1} k^{2}\left|\tau_{k}(q)-\tau_{k}\left(q_{0}\right)\right|^{2}\right)^{1 / 2}+\frac{C}{n^{2}}\left(\sum_{k \geq 1}\left|\gamma_{k}(q)^{2}-\gamma_{k}\left(q_{0}\right)^{2}\right|\right)^{1 / 2} .
\end{aligned}
$$

In view of (A.2) and $\sup _{\lambda \in \underset{n \geq 1}{\cup} \Gamma_{n}} e^{\mid \operatorname{Im} \sqrt{\lambda \mid}}<\infty$ one concludes that there exists $C \geq 1$ such that for $\phi \in I_{3 / 2}$ and $n \geq 1$

$$
\sup _{\lambda \in \Gamma_{n}}|\phi(\lambda)| \leq \frac{C}{n^{2}}\|\phi\|_{I_{3 / 2}} .
$$

Combining the estimates (A.6)-(A.6 $\left.6^{\prime \prime \prime}\right)$ we deduce from (A.5) that there exist $N \geq 1$ and $C \geq 1$ such that for $n \geq N, q \in V$ the claimed estimate for $n\left\|A_{n}(q)-A_{n}\left(q_{0}\right)\right\|_{I_{3 / 2}^{*}}$ holds.

Since $A_{n}(q)$ is continuous on $V \subseteq L_{0}^{2}\left(S^{1} ; \mathbb{C}\right)$ with values in $I_{3 / 2}^{*}$, Lemma A.1 leads to (use the asymptotics of the eigenvalues $\lambda_{j}(q)$ to estimate the right hand side of the formula in Lemma A.3).

Corollary A.2. Let $q_{0} \in L_{0}^{2}\left(S^{1} ; \mathbb{C}\right)$ be real valued. Then, for any $\epsilon>0$, there exists a neighborhhood $U \equiv U_{\epsilon} \subseteq V$ of $q_{0}$ in $L_{0}^{2}\left(S^{1} ; \mathbb{C}\right)$ so that for $q$ in $U$

$$
\sum_{n \geq 1} n^{2}\left\|A_{n}(q)-A_{n}\left(q_{0}\right)\right\|_{I_{3 / 2}^{*}}^{2} \leq \epsilon
$$

Theorem A.3. Let $q_{0} \in L_{0}^{2}\left(S^{1} ; \mathbb{C}\right)$ be a real valued potential. Then there exists a neighborhood $U$ of $q_{0}$ in $L_{0}^{2}\left(S^{1} ; \mathbb{C}\right)$ with the following properties:

(i) For any $q$ in $U,\left(n A_{n}(q)\right)_{n \geq 1}$ is a Riesz basis of $I_{3 / 2}^{*}$; 
(ii) there exists a uniquely determined sequence $\left(\frac{1}{n} 1_{n}(\cdot, q)\right)_{n \geq 1}$ in $I_{3 / 2}$, each of the elements $\frac{1}{n} 1_{n}(\cdot, q)$ depending analytically on $q \in U$, so that

$$
\left(n A_{n}(q)\right)\left(\frac{1}{k} 1_{k}(\cdot, q)\right)=\delta_{n k}
$$

(iii) for any $q$ in $U,\left(\frac{1}{n} 1_{n}(\cdot, q)\right)_{n \geq 1}$ is a Riesz basis of $I_{3 / 2}$.

Proof. Let $U$ be a neighborhood as in Corollary A.2. Since $\left(n A_{n}\left(q_{0}\right)\right)_{n \geq 1}$ is a Riesz basis of $I_{3 / 2}^{*}$, we can define a linear bounded map $T(q): I_{3 / 2}^{*} \rightarrow I_{3 / 2}^{*}$ by setting $T(q)\left(n A_{n}\left(q_{0}\right)\right):=n A_{n}(q)$. By Corollary A.2, $I d-T(q)$ is a Hilbert-Schmidt operator and by choosing $0<\epsilon<1$ sufficiently small we conclude from Corollary A.2 that $\|I d-T(q)\|_{H S}<1$ for any $q$ in $U$. (Given a linear operator $A: H \rightarrow H$ on a Hilbert space $H$ with an orthonormal basis $\left(e_{j}\right)_{j \geq 1},\|A\|_{H S}$ denotes the Hilbert-Schmidt norm $\|A\|_{H S}=\left(\sum_{j, k}\left|\left\langle e_{j}, A e_{k}\right\rangle\right|^{2}\right)^{1 / 2}$.) In particular $T(q)$ is an invertible, bounded linear operator and thus $\left(T(q)\left(n A_{n}\left(q_{0}\right)\right)\right)_{n \geq 1}$ is a Riesz basis of $I_{3 / 2}^{*}$ as well. Using the same symbols as McKean-Trubowitz [MT2], we denote by $\left(\frac{1}{n} 1_{n}\left(\lambda, q_{0}\right)\right)_{n \geq 1}$ the Riesz basis of $I_{3 / 2}$ uniquely determined by $n A_{n}\left(q_{0}\right)\left(\frac{1}{k} 1_{k}\left(\cdot, q_{0}\right)\right)=\delta_{n k}$.

Let $\frac{1}{n} 1_{n}(\cdot, q):=\left(T(q)^{-1}\right)^{*}\left(\frac{1}{n} 1_{n}\left(\cdot, q_{0}\right)\right)$ where $\left(T(q)^{-1}\right)^{*}$ denotes the adjoint of $T(q)^{-1}$. One verifies that $n A_{n}(q)\left(\frac{1}{k} 1_{k}(\cdot, q)\right)=\delta_{n k}$. It remains to prove that the $1_{n}(\cdot, q)$ 's depend analytically on $q \in U$. Denote by $\mathcal{L}_{H S}\left(I_{3 / 2}^{*}, I_{3 / 2}^{*}\right)$ the Hilbert space of Hilbert-Schmidt operators on $I_{3 / 2}^{*}$ with inner product

$$
\langle R, S\rangle_{H S}:=\sum_{i, j} R_{i j} \overline{S_{j i}}
$$

where $R_{i j}=\left\langle R e_{i}, e_{j}\right\rangle$ are the coefficients of $R$ with respect to an orthonormal basis $\left(e_{i}\right)_{i \geq 1}$ of $I_{3 / 2}^{*}$. From the analyticity of $A_{n}(q)$ it follows that the coefficients of $I d-T(q)$ are analytic on $U$. By Corollary A.2, $I d-T(q)$ is bounded on $U$ in $\mathcal{L}_{H S}\left(I_{3 / 2}^{*}, I_{3 / 2}^{*}\right)$ and therefore, $I d-T(q)$ is analytic (cf. e.g. $\left[\mathbf{P T}\right.$, Appendix A]). As $\left\|(I d-T(q))^{-1}\right\|_{H S}<1$,

$$
T(q)^{-1}=(I d-(I d-T(q)))^{-1}=\sum_{k \geq 0}(I d-T(q))^{k}
$$

where the series converges in $\mathcal{L}_{H S}\left(I_{3 / 2}^{*}, I_{3 / 2}^{*}\right)$. Therefore $T(q)^{-1}$ is analytic which implies that $\frac{1}{n} 1_{n}(\cdot, q)=\left(T(q)^{-1}\right)^{*}\left(\frac{1}{n} 1_{n}\left(\cdot, q_{0}\right)\right)$ is analytic on $U$ with values in $I_{3 / 2}$ for any $n \geq 1$. 
Remark. For $q=0$, the Riesz basis $\left(n A_{n}(q)\right)_{n \geq 1}$ of $I_{3 / 2}^{*}$ and $\left(\frac{1}{n} 1_{n}(\cdot, q)\right)_{n \geq 1}$ of $I_{3 / 2}$ are given by

$$
n A_{n}(0)=(-1)^{n} 2 n^{2} \pi^{2} \delta_{\left(n^{2} \pi^{2}\right)}
$$

and

$$
\frac{1}{n} 1_{n}(\lambda, 0)=\frac{\sin \sqrt{\lambda}}{\sqrt{\lambda}\left(\lambda-n^{2} \pi^{2}\right)} .
$$

\section{A.2 Rouché's theorem.}

Denote by $I_{-1 / 2}$ the complex Hilbert space of entire functions $\phi$ of order $\leq 1 / 2$ and type $\leq 1$ with

$$
\|\phi\|_{I_{-1 / 2}}^{2}:=\int_{0}^{\infty}|\phi(\lambda)|^{2} \frac{d \lambda}{\sqrt{\lambda}}<\infty .
$$

The inner product in $I_{-1 / 2}$ is given by

$$
\langle\phi, \psi\rangle:=\int_{0}^{\infty} \phi(\lambda) \overline{\psi(\lambda)} \frac{d \lambda}{\sqrt{\lambda}}<\infty
$$

By the Paley-Wiener theorem

$$
|\phi(\lambda)| \leq\|\phi\|_{I_{-1 / 2}} e^{\mid \operatorname{Im} \sqrt{\lambda \mid}}
$$

Denote by $I_{-1 / 2}^{*}$ the Hilbert space dual to $I_{-1 / 2}$. Notice that the identity embedding $I_{3 / 2} \hookrightarrow I_{-1 / 2}$ is continuous. As in Section A.1, denote by $V$ a neighborhood of a real valued potential $q_{0} \in L_{0}^{2}$ with the property (A.3). For $q$ in $V$, the functionals $A_{n}(q)$ introduced in (A.4) extend to linear bounded functionals on $I_{-1 / 2}, A_{n}(q): I_{-1 / 2} \rightarrow \mathbb{C}$. Using (A.7) and the definition of $A_{n}(q)$ one concludes that there exists $C>1$ such that $\frac{1}{C} \leq\left\|\frac{1}{n} A_{n}(q)\right\|_{I_{-1 / 2}^{*}} \leq$ $C$ for $q$ in $V$. By the same arguments used in the proof of Lemma A.1 one obtains

Lemma A.1'. There exist $C \geq 1$ and $N \geq 1$ such that for $n \geq N$ and $q \in V$

$$
\begin{aligned}
& \frac{1}{n}\left\|A_{n}(q)-A_{n}\left(q_{0}\right)\right\|_{I_{-1 / 2}^{*}} \\
& \leq C\left(\left|\gamma_{n}(q)-\gamma_{n}\left(q_{0}\right)\right|^{2}+\left|\tau_{n}(q)-\tau_{n}\left(q_{0}\right)\right|\right)
\end{aligned}
$$




$$
\begin{aligned}
& +\frac{C}{n^{2}}\left(\left|\lambda_{0}(q)-\lambda_{0}\left(q_{0}\right)\right|+\left(\sum_{k \geq 1} k^{2}\left|\tau_{k}(q)-\tau_{k}\left(q_{0}\right)\right|^{2}\right)^{1 / 2}\right. \\
& \left.\quad+\left(\sum_{k \geq 1}\left|\gamma_{k}(q)^{2}-\gamma_{k}\left(q_{0}\right)^{2}\right|\right)^{1 / 2}\right) .
\end{aligned}
$$

Next we need to estimate the $I_{-1 / 2}$-norm of the functions $n 1_{n}\left(\lambda, q_{0}\right)$ for a real valued potential $q_{0}$.

Lemma A.4. Let $q_{0} \in L_{0}^{2}\left(S^{1} ; \mathbb{R}\right)$. Then there exists $C^{\prime}>0$ such that

$$
\left\|n 1_{n}\left(\cdot, q_{0}\right)\right\|_{I_{-1 / 2}} \leq C^{\prime} \quad(n \geq 1) .
$$

Proof. It follows from the Paley-Wiener theorem that the Dirac measures $\sqrt{\pi} \delta_{0},\left(\sqrt{2 \pi} \delta_{n^{2} \pi^{2}}\right)_{n \geq 1}$ are an orthonormal basis of $I_{-1 / 2}^{*}$ (Kotelnikov theorem; cf. also Remark after Theorem A.3). Therefore

$$
\left\|j 1_{j}\left(\cdot, q_{0}\right)\right\|_{I_{-1 / 2}}^{2}=\pi\left|j 1_{j}\left(0, q_{0}\right)\right|^{2}+2 \pi \sum_{n \geq 1}\left|j 1_{j}\left(n^{2} \pi^{2}, q_{0}\right)\right|^{2} .
$$

Further recall that, as $q_{0}$ is real valued, ([MT1])

$$
j 1_{j}\left(\lambda, q_{0}\right)=C_{j} \prod_{k \neq j} \frac{\mu_{k}^{(j)}-\lambda}{k^{2} \pi^{2}}
$$

where $\lambda_{2 k-1}\left(q_{0}\right) \leq \mu_{k}^{(j)} \leq \lambda_{2 k}\left(q_{0}\right)$ and $\frac{1}{C} \leq C_{j} \leq C$ for all $j \geq 1$ for some constant $C \geq 1$.

For $1 \leq n \neq j$, write

$$
\begin{aligned}
j 1_{j}\left(n^{2} \pi^{2}, q_{0}\right) & =C_{j} \prod_{k \neq j} \frac{\mu_{k}^{(j)}-n^{2} \pi^{2}}{k^{2} \pi^{2}} \\
& =C_{j}\left(\prod_{k \neq n, j} \frac{\mu_{k}^{(j)}-n^{2} \pi^{2}}{k^{2} \pi^{2}}\right) \frac{\tau_{j}-n^{2} \pi^{2}}{j^{2} \pi^{2}} \frac{j^{2} \pi^{2}}{\tau_{j}-n^{2} \pi^{2}} \cdot \frac{\mu_{n}^{(j)}-n^{2} \pi^{2}}{n^{2} \pi^{2}} .
\end{aligned}
$$

Notice that there exists $C \geq 1$ (cf. e.g. [PT, Appendix E, Lemma 3]) such that for $j, n \geq 1$ with $j \neq n$

$$
\left|\left(\prod_{k \neq n, j} \frac{\mu_{k}^{(j)}-n^{2} \pi^{2}}{k^{2} \pi^{2}}\right) \frac{\tau_{j}-n^{2} \pi^{2}}{j^{2} \pi^{2}}\right| \leq C .
$$


This implies that there exists $C \geq 1$ such that for $j \neq n, j, n \geq 1$,

$$
\left|j 1_{j}\left(n^{2} \pi^{2}, q_{0}\right)\right| \leq C \frac{j^{2}}{\left|j^{2}-n^{2}\right|} \frac{\mu_{n}^{(j)}-n^{2} \pi^{2}}{n^{2} \pi^{2}} .
$$

Similarly, there exists $C \geq 1$ such that for $j \geq 1$

$$
\left|j 1_{j}\left(j^{2} \pi^{2}, q_{0}\right)\right| \leq C \quad \text { and } \quad\left|j 1_{j}\left(0, q_{0}\right)\right| \leq C .
$$

Notice that for $1 \leq|n-j| \leq j / 2, \frac{j^{2}}{\left|j^{2}-n^{2}\right|} \frac{1}{n^{2}} \leq \frac{C}{\left|j^{2}-n^{2}\right|}$ and for $|n-j|>j / 2$, $\frac{j^{2}}{j^{2}-n^{2}} \frac{1}{n^{2}} \leq C n^{2}$. Therefore we conclude that there exists $C \geq 1$ such that for any $j \geq 1$,

$$
\sum_{n \geq 0}\left|j 1_{j}\left(n^{2} \pi^{2}, q_{0}\right)\right|^{2} \leq 2 C+C \sum_{n \neq j}\left|\mu_{n}^{(j)}-n^{2} \pi^{2}\right|^{2} .
$$

As $\lambda_{2 n-1}\left(q_{0}\right) \leq \mu_{n}^{(j)} \leq \lambda_{2 n}\left(q_{0}\right)$ the claimed result then follows from the asymptotics of the eigenvalues.

In order to apply Rouché's theorem we need to prove that $\mid \frac{1}{j} 1_{j}(\lambda, q)-$ $\frac{1}{j} 1_{j}\left(\lambda, q_{0}\right)|<| \frac{1}{j} 1_{j}\left(\lambda, q_{0}\right) \mid$ for $\lambda \in \Gamma_{n}$ and $q \in U$. Notice that the difference $\psi_{j}(\lambda, q):=\frac{1}{j} 1_{j}(\lambda, q)-\frac{1}{j} 1_{j}\left(\lambda, q_{0}\right)$ is in $I_{3 / 2}$ and therefore, by Theorem A.3, can be written as

$$
\psi_{j}(\lambda, q)=\sum_{n \geq 1} n A_{n}\left(q_{0}\right)\left(\psi_{j}(\cdot, q)\right) \frac{1}{n} 1_{n}\left(\lambda, q_{0}\right) .
$$

For any $\lambda \in \mathbb{C}$, the Dirac measure $\delta_{\lambda}$ is in $I_{-1 / 2}^{*}$ and $\left|\delta_{\lambda}(\psi)\right| \leq$ $\|\psi\|_{I_{-1 / 2}} e^{\mid \operatorname{Im} \sqrt{\lambda \mid}}$ (cf. A.7). Introduce $\beta:=\sup _{\lambda \in \cup_{1}^{\infty} \Gamma_{k}} e^{\mid \operatorname{Im} \sqrt{\lambda \mid}}$. Then $\beta<\infty$ and

$$
\sup _{\lambda \in \cup_{1}^{\infty} \Gamma_{k}}\left|\psi_{j}(\lambda, q)\right| \leq \beta\left\|\psi_{j}(\cdot, q)\right\|_{I_{-1 / 2}} .
$$

Further, by Lemma A.4, there exists $C^{\prime} \geq 1$ such that for $j \geq 1$,

$$
\begin{aligned}
& \left\|\psi_{j}(\cdot, q)\right\|_{I_{-1 / 2}} \\
& \leq \sum_{n \geq 1}^{\infty}\left|n A_{n}\left(q_{0}\right)\left(\psi_{j}(\cdot, q)\right)\right|\left\|\frac{1}{n} 1_{n}\left(\cdot, q_{0}\right)\right\|_{I_{-1 / 2}} \\
& \leq C^{\prime} \sum_{n \geq 1}^{\infty} \frac{1}{n^{2}}\left|n A_{n}\left(q_{0}\right)\left(\psi_{j}(\cdot, q)\right)\right| .
\end{aligned}
$$

Rewrite $n A_{n}\left(q_{0}\right)\left(\psi_{j}(\cdot, q)\right)$, using the definition of $\psi_{j}$,

$$
n A_{n}\left(q_{0}\right)\left(\psi_{j}(\cdot, q)\right)=n A_{n}\left(q_{0}\right)\left(\frac{1}{j} 1_{j}(\cdot, q)\right)-\delta_{n j}
$$




$$
\begin{aligned}
& =\left(n A_{n}\left(q_{0}\right)-n A_{n}(q)\right)\left(\frac{1}{j} 1_{j}(\cdot, q)\right) \\
& =\left(n A_{n}\left(q_{0}\right)-n A_{n}(q)\right)\left(\frac{1}{j} 1_{j}\left(\cdot, q_{0}\right)\right)+\left(n A_{n}\left(q_{0}\right)-n A_{n}(q)\right)\left(\psi_{j}(\cdot, q)\right)
\end{aligned}
$$

to conclude that

$$
\begin{aligned}
& \sum_{n \geq 1} \frac{1}{n^{2}}\left|n A_{n}\left(q_{0}\right)\left(\psi_{j}(\cdot, q)\right)\right| \\
& \leq \sum_{n \geq 1} \frac{1}{n^{2}}\left\|n A_{n}(q)-n A_{n}\left(q_{0}\right)\right\|_{I_{-1 / 2}^{*}}\left\|\frac{1}{j} 1_{j}\left(\cdot, q_{0}\right)\right\|_{I_{-1 / 2}} \\
& \quad+\sum_{n \geq 1} \frac{1}{n^{2}}\left\|n A_{n}(q)-n A_{n}\left(q_{0}\right)\right\|_{I_{-1 / 2}^{*}}\left\|\psi_{j}(\cdot, q)\right\|_{I_{-1 / 2}} .
\end{aligned}
$$

Apply Lemma A.1' in a way similar as Lemma A.1 to Corollary A.2 to conclude that for any $0<\epsilon \leq \frac{1}{2}$ there exists a neighborhood $U \equiv U_{\epsilon} \subset V$ of $q_{0}$ in $L_{0}^{2}\left(S^{1} ; \mathbb{C}\right)$, such that, for $q \in U$,

$$
\sum_{n \geq 1}\left\|\frac{1}{n} A_{n}(q)-\frac{1}{n} A_{n}\left(q_{0}\right)\right\|_{I_{-1 / 2}^{*}} \leq \epsilon .
$$

Together, the two estimates above lead to

$$
\left\|\psi_{j}(\cdot, q)\right\|_{I_{-1 / 2}} \leq \epsilon\left\|\frac{1}{j} 1_{j}\left(\cdot, q_{0}\right)\right\|_{I_{-1 / 2}}+\frac{1}{2}\left\|\psi_{j}(\cdot, q)\right\|_{I_{-1 / 2}} .
$$

Together with Lemma A.4, this implies $\left\|\psi_{j}(\cdot, q)\right\|_{I_{-1 / 2}} \leq 2 \epsilon\left\|\frac{1}{j} 1_{j}\left(\cdot, q_{0}\right)\right\|_{I_{-1 / 2}} \leq$ $2 \epsilon \frac{C^{\prime}}{j^{2}}$. Taking into account (A.9) and the definition of $\psi_{j}$ this estimate leads to

$$
\sup _{\lambda \in \cup_{1}^{\infty} \Gamma_{k}}\left|\frac{1}{j} 1_{j}(\lambda, q)-\frac{1}{j} 1_{j}\left(\lambda, q_{0}\right)\right| \leq \beta 2 C^{\prime} \frac{\epsilon}{j^{2}}
$$

for any $q \in U$ and $j \geq 1$.

To be able to apply Rouché's theorem we also need to estimate $\frac{1}{j} 1_{j}\left(\lambda, q_{0}\right)$ from below for $\lambda \in \Gamma_{n}$. As $q_{0}$ is real valued, $\frac{1}{j} 1_{j}\left(\lambda, q_{0}\right)$ has product representation (A.8) and in view of the definition of the circles $\Gamma_{n}(n \geq 1)$, we conclude that for any integer $1 \leq N<\infty$ there exists $\rho_{N}>0$ such that for any $j \geq 1$

$$
\inf _{\lambda \in \cup_{1}^{N} \Gamma_{n}}\left|\frac{1}{j} 1_{j}\left(\lambda, q_{0}\right)\right| \geq \rho_{N} \frac{1}{j^{2}}
$$


Combining (A.10) and (A.11) we obtain

$$
\left|\frac{1}{j} 1_{j}(\lambda, q)\right| \geq\left|\frac{1}{j} 1_{j}\left(\lambda, q_{0}\right)\right|-\left|\frac{1}{j} 1_{j}(\lambda, q)-\frac{1}{j} 1_{j}\left(\lambda, q_{0}\right)\right| \geq \frac{\rho_{N}-\beta 2 C^{\prime} \epsilon}{j^{2}}
$$

for $\lambda \in \bigcup_{1}^{N} \Gamma_{n}, q \in U$ and $j \geq 1$.

Theorem A.5. Let $q_{0} \in L_{0}^{2}\left(S^{1} ; \mathbb{C}\right)$ be real valued, $1 \leq N<\infty$ an integer and $0<K \leq K\left(q_{0}\right)$. Then there exists a bounded neighborhood $U_{N, K}$ of $q_{0}$ in $L_{0}^{2}\left(S^{1} ; \mathbb{C}\right)$ with the following properties:

(i) The statements of Theorem A.3 hold and (A.3) is valid;

(ii) for any $q \in U_{N, K}$ and $j \geq 1$, the function $\frac{1}{j} 1_{j}(\lambda, q)$ has precisely one zero, denoted by $\mu_{k}^{(j)}(q)$, inside the circle $\Gamma_{k}=\Gamma_{k}(K)$ for $1 \leq k \leq$ $N, k \neq j$. In addition, if $1 \leq j \leq N, \frac{1}{j} 1_{j}(\lambda, q)$ has no zero inside $\Gamma_{j}(K)$;

(iii) the zeroes $\mu_{k}^{(j)}(q)$ are analytic functions of $q$ on $U_{N, K}$.

Proof. For ease of writing, from now on, we do not always indicate the dependence on the choice of $K$. Choose $\epsilon>0$ and a neighborhood $U \equiv U_{\epsilon, N}$ of $q_{0}$ in $L_{0}^{2}\left(S^{1} ; \mathbb{C}\right)$ sufficiently small such that the statements of Theorem A.3 and (A.10) are valid and such that $\beta C \epsilon \leq \frac{1}{2} \rho_{N}$ where the product $\beta C$ is as in (A.10) and $\rho_{N}$ is given as in (A.11). Then

$$
\sup _{\lambda \in \cup_{1}^{N} \Gamma_{n}}\left|\frac{1}{j} 1_{j}(\lambda, q)-\frac{1}{j} 1_{j}\left(\lambda, q_{0}\right)\right| \leq \frac{1}{2} \inf _{\lambda \in \cup_{1}^{N} \Gamma_{n}}\left|\frac{1}{j} 1_{j}\left(\lambda, q_{0}\right)\right|
$$

for any $j \geq 1$ and $q$ in $U$. Further notice that, due to the product representation (A.8), statement (ii) holds for $q=q_{0}$. Therefore, by Rouché's theorem, (ii) follows. Statement (iii) is a consequence of Cauchy's integral formula

$$
\mu_{k}^{(j)}(q)=\frac{1}{2 \pi i} \int_{\Gamma_{k}} \lambda \frac{\frac{d}{d \lambda} 1_{j}(\lambda, q)}{1_{j}(\lambda, q)} d \lambda
$$

together with the analyticity of $1_{j}(\lambda, q)$ (cf. Theorem A.3).

\section{A.3. Contraction mapping.}

To localize the zeroes of the $1_{j}(\lambda, q)$ outside the circles $\Gamma_{n}(1 \leq n \leq N)$, we consider the following system (cf. notation of Section 2 or (A.16) below)

$$
\xi_{k}^{(j)}(q)=\frac{\gamma_{k}(q)}{2} \frac{\int_{0}^{1} \frac{t d t}{\sqrt{1-t^{2}}}\left(C_{j k}(t)-C_{j k}(-t)\right)}{\int_{0}^{1} \frac{d t}{\sqrt{1-t^{2}}}\left(C_{j k}(t)+C_{j k}(-t)\right)} \quad(k \geq N+1, k \neq j)
$$


where $C_{j k}(t)=C_{j k}\left(t, q, \xi^{(j)}(q)\right)$ and $\mu_{k}^{(j)}(q)=\tau_{k}(q)+\xi_{k}^{(j)}(q)$. Let's first outline how we obtain a solution of (A.12) by using a fixed point argument:

Let $U$ be a neighborhood of $q_{0}$ in $L_{0}^{2}\left(S^{1} ; \mathbb{C}\right)$ with the properties of Theorem A.3. Let $N \geq 1$ and chose arbitrarily $q \in U$ and $j \geq 1$. (We will choose $N$ later sufficiently large, but $N$ will be independent of $q$ and $j$.) Let $\mathbb{N} \backslash\{j\}=$ $\mathcal{A} \cup \mathcal{B}$ where $\mathcal{A} \equiv \mathcal{A}_{N j}:=\{1 \leq k \leq N ; k \neq j\}$ and $\mathcal{B} \equiv \mathcal{B}_{N_{j}}:=(\mathbb{N} \backslash\{j\}) \backslash \mathcal{A}$.

Elements $\eta=\left(\eta_{k}\right)_{k \neq j}$ in $\ell^{2}(\mathbb{N} \backslash\{j\} ; \mathbb{C})$ are decomposed $\eta=\left(\eta_{\mathcal{A}}, \eta_{\mathcal{B}}\right)$ with $\eta_{\mathcal{A}}=\left(\eta_{k}\right)_{k \in \mathcal{A}} \in \ell^{2}(\mathcal{A} ; \mathbb{C})$ and $\eta_{\mathcal{B}}=\left(\eta_{k}\right)_{k \in \mathcal{B}} \in \ell^{2}(\mathcal{B} ; \mathbb{C})$. Given $q \in U$, denote by $\mathcal{C}_{\mathcal{A}}$ and $\mathcal{C}_{\mathcal{B}}$ the Hilbert cubes (with induced strong topology) $\mathcal{C}_{\mathcal{A}} \equiv$ $\mathcal{C}_{\mathcal{A}_{N j}}:=\left\{\eta_{\mathcal{A}} ;\left|\eta_{k}\right| \leq \gamma_{k}\left(q_{0}\right)+\frac{1}{k} K\right.$ for $\left.k \in \mathcal{A}\right\}$ and $\mathcal{C}_{\mathcal{B}} \equiv \mathcal{C}_{\mathcal{B}_{N j}}:=\left\{\eta_{\mathcal{B}} ;\left|\eta_{k}\right| \leq\right.$ $\frac{1}{2}\left|\gamma_{k}(q)\right|+\min \left(K, \frac{1}{2}\left|\gamma_{k}(q)\right|\right)$ for $\left.k \in \mathcal{B}\right\}$. (Cf. (A.3) for the constant K.)

Introduce the map $T=T_{q}^{(j)}: \mathcal{C}_{\mathcal{A}} \times \mathcal{C}_{\mathcal{B}} \rightarrow \ell^{2}(\mathcal{B} ; \mathbb{C})$ where the components of $T\left(\eta_{\mathcal{A}}, \eta_{\mathcal{B}}\right)$ are given by the righthand side of (A.12)

$$
T\left(\eta_{\mathcal{A}}, \eta_{\mathcal{B}}\right)=\left(\frac{\gamma_{k}(q)}{2} \frac{\int_{0}^{1} \frac{t d t}{\sqrt{1-t^{2}}}\left(C_{j k}(t)-C_{j k}(-t)\right)}{\int_{0}^{1} \frac{d t}{\sqrt{1-t^{2}}}\left(C_{j k}(t)+C_{j k}(-t)\right)}\right)_{k \in \mathcal{B}}
$$

with $C_{j k}(t)=C_{j k}\left(t, q, \eta_{\mathcal{A}}, \eta_{\mathcal{B}}\right)$. Using the same arguments as in Section 2 one shows that there exist a (sufficiently small) neighborhood $U$ of $q_{0}$ in $L_{0}^{2}\left(S^{1} ; \mathbb{C}\right)$ and $N \geq 1$ sufficiently large (independent of $q, j \geq 1$ ) so that $T\left(\eta_{\mathcal{A}}, \eta_{\mathcal{B}}\right) \in \mathcal{C}_{\mathcal{B}}$ (cf. Proposition A.6 below). Moreover, for any $\eta_{\mathcal{A}} \in \mathcal{C}_{\mathcal{A}}$, $T\left(\eta_{\mathcal{A}}, \cdot\right): \mathcal{C}_{\mathcal{B}} \rightarrow \mathcal{C}_{\mathcal{B}}$ is a contraction (cf. Proposition A.7 below). Therefore there exists a unique element in $\mathcal{C}_{\mathcal{B}}$, denoted by $\kappa\left(\eta_{\mathcal{A}}\right)$, satisfying

$$
\kappa\left(\eta_{\mathcal{A}}\right)=T\left(\eta_{\mathcal{A}}, \kappa\left(\eta_{\mathcal{A}}\right)\right)
$$

In the remainder of this section we prove the statements claimed above.

Proposition A.6. There exist a (sufficiently small) neighborhood $U$ of $q_{0}$ in $L_{0}^{2}\left(S^{1} ; \mathbb{C}\right)$ and $N \geq 1$ so that for $q$ in $U$ and $j \geq 1$ the map $T=$ $T_{q}^{(j)}: \mathcal{C}_{\mathcal{A}} \times \mathcal{C}_{\mathcal{B}} \rightarrow \ell^{2}(\mathcal{B} ; \mathbb{C})$ satisfies the following estimate for $k \in \mathcal{B}$ and any $\left(\eta_{\mathcal{A}}, \eta_{\mathcal{B}}\right) \in \mathcal{C}_{\mathcal{A}} \times \mathcal{C}_{\mathcal{B}}$

$$
\left|T\left(\eta_{\mathcal{A}}, \eta_{\mathcal{B}}\right)_{k}\right| \leq C \frac{\left|\gamma_{k}(q)\right|^{2}}{k} \leq \frac{1}{2}\left|\gamma_{k}(q)\right|+\min \left(K,\left|\gamma_{k}(q)\right|\right)
$$

where $C>0$ is independent of $q \in U, j \geq 1$ and $k \in \mathcal{B}$.

Proof. Recall that $T\left(\eta_{\mathcal{A}}, \eta_{\mathcal{B}}\right)$ is given by

$$
T\left(\eta_{\mathcal{A}}, \eta_{\mathcal{B}}\right)=\left(\frac{\gamma_{k}(q)}{2} \frac{\int_{0}^{1} \frac{t d t}{\sqrt{1-t^{2}}}\left(C_{j k}(t)-C_{j k}(-t)\right)}{\int_{0}^{1} \frac{d t}{\sqrt{1-t^{2}}}\left(C_{j k}(t)+C_{j k}(-t)\right)}\right)_{k \in \mathcal{B}}
$$


As in Section 2, we write $C_{j k}(t) \equiv C_{j k}\left(t, q, \eta_{\mathcal{A}}, \eta_{\mathcal{B}}\right)$ as a product of the form

$$
C_{j k}(t)=\sqrt{\frac{\Delta_{0}(0)}{\Delta_{0}(t)}} \sqrt{\frac{\Delta_{j}(0)}{\Delta_{j}(t)}} \frac{A_{j k}(t)}{A_{j k}(0)} \frac{B_{j k}(t)}{B_{j k}(0)} .
$$

By the same proof as for Lemma 2.3-Lemma 2.5 one shows that there exists a neighborhood $U$ of $q_{0}$ in $L_{0}^{2}\left(S^{1} ; \mathbb{C}\right)$ and $C>0$ independent of $j, k$ and $q \in U$ so that for $q \in U, k \neq j$

$$
\begin{aligned}
\sup _{|t| \leq 1}\left|\frac{A_{j k}(t)}{A_{j k}(0)}-1\right| & \leq C\left|\gamma_{k}(q)\right| \sum_{|\alpha-k| \geq \frac{k}{2}} \frac{\left|\gamma_{k}(q)\right|}{\alpha^{4}}+C\left|\gamma_{k}(q)\right| \sum_{|\alpha-k| \leq \frac{k}{2}} \frac{\left|\gamma_{\alpha}(q)\right|}{\alpha^{2}} \\
& \leq C \frac{\left|\gamma_{k}(q)\right|}{k}, \\
\sup _{|t| \leq 1}\left|\frac{B_{j k}(t)}{B_{j k}(0)}-1\right| & \leq \frac{C\left|\gamma_{k}(q)\right|}{k^{3}}\left(\sum_{|\alpha-k| \leq \frac{k}{2}}\left|\gamma_{\alpha}\right|^{2}+\frac{1}{k^{3}}\right) \\
& \leq C \frac{\left|\gamma_{k}(q)\right|}{k^{3}}
\end{aligned}
$$

and

$$
\begin{aligned}
& \sup _{|t| \leq 1}\left|\left(\frac{\Delta_{0}(0)}{\Delta_{0}(t)}\right)^{1 / 2}-1\right| \leq C \frac{\left|\gamma_{k}(q)\right|}{k^{2}} \\
& \sup _{|t|<1}\left|\left(\frac{\Delta_{j}(0)}{\Delta_{j}(t)}\right)^{1 / 2}-1\right| \leq C \frac{\left|\gamma_{k}(q)\right|}{k} .
\end{aligned}
$$

We proceed as in the proof of Theorem 2.7 and write

$$
\begin{aligned}
C_{j k}(t)= & \left(\frac{\Delta_{j}(0)}{\Delta_{j}(t)}\right)^{1 / 2}+\left(\frac{\Delta_{j}(0)}{\Delta_{j}(t)}\right)^{1 / 2}\left(\left(\frac{\Delta_{0}(0)}{\Delta_{0}(t)}\right)^{1 / 2}-1\right) \\
& +\left(\frac{\Delta_{j}(0)}{\Delta_{j}(t)}\right)^{1 / 2}\left(\frac{\Delta_{0}(0)}{\Delta_{0}(t)}\right)^{1 / 2}\left(\frac{B_{j k}(t)}{B_{j k}(0)}-1\right) \\
& +\left(\frac{\Delta_{j}(0)}{\Delta_{j}(t)}\right)^{1 / 2}\left(\frac{\Delta_{0}(0)}{\Delta_{0}(t)}\right)^{1 / 2} \frac{B_{j k}(t)}{B_{j k}(0)}\left(\frac{A_{j k}(t)}{A_{j k}(0)}-1\right) .
\end{aligned}
$$

Then for $|t| \leq 1,\left(\eta_{\mathcal{A}}, \eta_{\mathcal{B}}\right) \in \mathcal{C}_{\mathcal{A}} \times \mathcal{C}_{\mathcal{B}}$

$$
\begin{aligned}
& \left|\frac{\Delta_{j}(0)}{\Delta_{j}(t)}\right|^{1 / 2} \leq 1+C \frac{\left|\gamma_{k}(q)\right|}{k}, \\
& \left|\left(\frac{\Delta_{j}(0)}{\Delta_{j}(t)}\right)^{1 / 2}\left(\left(\frac{\Delta_{0}(0)}{\Delta_{0}(t)}\right)^{1 / 2}-1\right)\right| \leq\left(1+C \frac{\left|\gamma_{k}(q)\right|}{k}\right) C \frac{\left|\gamma_{k}(q)\right|}{k^{2}}
\end{aligned}
$$




$$
\begin{aligned}
& \left|\left(\frac{\Delta_{j}(0)}{\Delta_{j}(t)}\right)^{1 / 2}\left(\frac{\Delta_{0}(0)}{\Delta_{0}(t)}\right)^{1 / 2}\left(\frac{B_{j k}(t)}{B_{j k}(0)}-1\right)\right| \\
& \leq\left(1+C \frac{\left|\gamma_{k}(q)\right|}{k}\right)\left(1+C \frac{\left|\gamma_{k}(q)\right|^{2}}{k^{2}}\right) C \frac{\left|\gamma_{k}(q)\right|}{k^{3}}
\end{aligned}
$$

and

$$
\begin{aligned}
& \left|\left(\frac{\Delta_{j}(0)}{\Delta_{j}(t)}\right)^{1 / 2}\left(\frac{\Delta_{0}(0)}{\Delta_{0}(t)}\right)^{1 / 2} \frac{B_{j k}(t)}{B_{j k}(0)}\left(\frac{A_{j k}(t)}{A_{j k}(0)}-1\right)\right| \\
& \leq\left(1+C \frac{\left|\gamma_{k}(q)\right|}{k}\right)\left(1+C \frac{\left|\gamma_{k}(q)\right|}{k^{2}}\right)\left(1+C \frac{\left|\gamma_{k}(q)\right|}{k^{3}}\right) C \frac{\left|\gamma_{k}(q)\right|}{k} .
\end{aligned}
$$

We conclude, similar as in the proof of Theorem 2.7 (and using that $\left|\eta_{k}\right| \leq\left|\gamma_{k}(q)\right|$ for $\left.k \in \mathcal{B}\right)$, that for $q \in U, j \geq 1,\left(\eta_{\mathcal{A}}, \eta_{\mathcal{B}}\right) \in \mathcal{C}_{\mathcal{A}} \times \mathcal{C}_{\mathcal{B}}$

$$
\left|T_{q}^{(j)}\left(\eta_{\mathcal{A}}, \eta_{\mathcal{B}}\right)_{k}\right| \leq C \frac{\left|\gamma_{k}(q)\right|^{2}}{k}
$$

Notice that $\left|\gamma_{k}(q)\right|^{2} \leq \sum_{\alpha \geq 1}\left|\gamma_{\alpha}(q)\right|^{2}$ is bounded on $U$. Therefore there exists $N \geq 1$ independent of $q \in U, j \geq 1$ such that for $k \geq N, q \in U$

$$
\frac{C\left|\gamma_{k}(q)\right|}{k} \leq 1 ; C \frac{\left|\gamma_{k}(q)\right|^{2}}{k} \leq \frac{\left|\gamma_{k}(q)\right|}{2}+K
$$

Notice that it follows from Proposition A.6 that the range of $T_{q}^{(j)}: \mathcal{C}_{\mathcal{A}} \times$ $\mathcal{C}_{\mathcal{B}} \rightarrow \ell^{2}(\mathcal{B} ; \mathbb{C})$ is contained in $\mathcal{C}_{\mathcal{B}}$

Proposition A.7. There exist a (sufficiently small) neighborhood $U$ of $q_{0}$ in $L_{0}^{2}\left(S^{1} ; \mathbb{C}\right)$ and $N \geq 1$ so that for $q \in U$ and $j \geq 1, T=T_{q}^{(j)}: \mathcal{C}_{\mathcal{A}} \times \mathcal{C}_{\mathcal{B}} \rightarrow \mathcal{C}_{\mathcal{B}}$ satisfies, for $\left(\eta_{\mathcal{A}}, \eta_{\mathcal{B}}\right) \in \mathcal{C}_{\mathcal{A}} \times \mathcal{C}_{\mathcal{B}}$

$$
\left\|T\left(\eta_{\mathcal{A}}^{\prime}, \eta_{\mathcal{B}}^{\prime}\right)-T\left(\eta_{\mathcal{A}}, \eta_{\mathcal{B}}\right)\right\|_{\ell^{2}(\mathcal{B} ; \mathbb{C})}^{2} \leq \frac{1}{4}\left(\left\|\eta_{\mathcal{A}}^{\prime}-\eta_{\mathcal{A}}\right\|^{2}+\left\|\eta_{\mathcal{B}}^{\prime}-\eta_{\mathcal{B}}\right\|^{2}\right)
$$

In particular, $T$ is continuous and, for any $\eta_{\mathcal{A}} \in \mathcal{C}_{\mathcal{A}}$ fixed, the map $T\left(\eta_{\mathcal{A}}, \cdot\right)$ : $\mathcal{C}_{\mathcal{B}} \rightarrow \mathcal{C}_{\mathcal{B}}$ is a contraction.

Proof. Notice that in the decomposition (A.16) of $C_{j k}(t)$ only the term $\frac{A_{j k}(t)}{A_{j k}(0)}$ depends on $\eta=\left(\eta_{\mathcal{A}}, \eta_{\mathcal{B}}\right)$. Recall that $A_{j k}(t)=\prod_{\alpha \in \mathbb{N} \backslash\{j, k\}}\left(1+\frac{\eta_{\alpha}}{\tau_{\alpha}-\lambda(t)}\right)$. Thus, with $D_{j k}(t) \equiv D_{j k}\left(t, q, \eta_{\mathcal{A}}^{\prime}, \eta_{\mathcal{B}}^{\prime}, \eta_{\mathcal{A}}, \eta_{\mathcal{B}}\right)$,

$$
D_{j k}(t):=\frac{A_{j k}\left(t, q, \eta_{\mathcal{A}}^{\prime}, \eta_{\mathcal{B}}^{\prime}\right)}{A_{j k}\left(0, q, \eta_{\mathcal{A}}^{\prime}, \eta_{\mathcal{B}}^{\prime}\right)}-\frac{A_{j k}\left(t, q, \eta_{\mathcal{A}}, \eta_{\mathcal{B}}\right)}{A_{j k}\left(0, q, \eta_{\mathcal{A}}, \eta_{\mathcal{B}}\right)}
$$




$$
\begin{aligned}
&=\sum_{\substack{\beta=1 \\
\beta \neq k, j}}^{\infty}\left(\prod_{\substack{\alpha<\beta \\
\alpha \neq k, j}} \frac{\left(1+\frac{\eta_{\alpha}^{\prime}}{\tau_{\alpha}-\lambda(t)}\right)}{1+\frac{\eta_{\alpha}^{\prime}}{\tau_{\alpha}-\tau_{k}}} \prod_{\substack{\alpha>\beta \\
\alpha \neq k, j}} \frac{\left(1+\frac{\eta_{\alpha}}{\tau_{\alpha}-\lambda(t)}\right)}{1+\frac{\eta_{\alpha}}{\tau_{\alpha}-\tau_{k}}}\right) \\
& \cdot\left(\frac{1+\frac{\eta_{\beta}^{\prime}}{\tau_{\beta}-\lambda(t)}}{1+\frac{\eta_{\beta}^{\prime}}{\tau_{\beta}-\tau_{k}}}-\frac{1+\frac{\eta_{\beta}}{\tau_{\beta}-\lambda(t)}}{1+\frac{\eta_{\beta}}{\tau_{\beta}-\tau_{k}}}\right) .
\end{aligned}
$$

Using the estimates in the proof of Proposition A.6 one shows that there exists $C>1$ independent of $q \in U, j \geq 1$ and $\left(\eta_{\mathcal{A}}^{\prime}, \eta_{\mathcal{B}}^{\prime}\right),\left(\eta_{\mathcal{A}}, \eta_{\mathcal{B}}\right) \in \mathcal{C}_{\mathcal{A}} \times \mathcal{C}_{\mathcal{B}}$ such that

$$
\left|D_{j k}(t)\right| \leq C \sum_{\beta \neq k, j} \frac{\left|\eta_{\beta}-\eta_{\beta}^{\prime}\right|}{\left|\beta^{2}-k^{2}\right|} .
$$

This leads to the estimate (for some $C>0$ independent of $q, j, \eta, \eta^{\prime}$ )

$$
\begin{aligned}
& \left\|T\left(\eta_{\mathcal{A}}^{\prime}, \eta_{\mathcal{B}}^{\prime}\right)-T\left(\eta_{\mathcal{A}}, \eta_{\mathcal{B}}\right)\right\|_{\ell^{2}(\mathcal{B} ; \mathbb{C})}^{2} \\
& \leq C \sum_{k \in \mathcal{B}}\left|\frac{\gamma_{k}(q)}{2}\right|^{2}\left|\sum_{\beta \neq k, j} \frac{\left|\eta_{\beta}-\eta_{\beta}^{\prime}\right|}{\left|\beta^{2}-k^{2}\right|}\right|^{2} \leq C \sum_{k \in \mathcal{B}}\left|\frac{\gamma_{k}(q)}{2}\right|^{2} \frac{1}{k^{2}}\left\|\eta-\eta^{\prime}\right\|^{2} .
\end{aligned}
$$

By choosing $N \geq 1$ sufficiently large, but independent of $j, q$ it follows that for any $q$ in $U$

$$
C \sum_{k \in \mathcal{B}}\left|\frac{\gamma_{k}(q)}{2}\right|^{2} \frac{1}{k^{2}} \leq \frac{1}{4}
$$

Corollary A.8. There exist a (sufficiently small) neighborhood $U$ of $q_{0}$ and $N \geq 1$ so that for $q \in U$ and for all $j \geq 1, T=T_{q}^{(j)}: \mathcal{C}_{\mathcal{A}} \times \mathcal{C}_{\mathcal{B}} \rightarrow \mathcal{C}_{\mathcal{B}}$ has for any $\eta_{\mathcal{A}} \in \mathcal{C}_{\mathcal{A}}$ a unique fixed point $\kappa\left(\eta_{\mathcal{A}}\right)=\kappa^{(j)}\left(\eta_{\mathcal{A}}, q\right) \in \mathcal{C}_{\mathcal{B}}$,

$$
T\left(\eta_{\mathcal{A}}, \kappa\left(\eta_{\mathcal{A}}\right)\right)=\kappa\left(\eta_{\mathcal{A}}\right)
$$

Moreover, for any $\eta_{\mathcal{A}}, \eta_{\mathcal{A}}^{\prime}$ in $\mathcal{C}_{\mathcal{A}}$,

$$
\left\|\kappa\left(\eta_{\mathcal{A}}^{\prime}\right)-\kappa\left(\eta_{\mathcal{A}}\right)\right\|_{\ell^{2}(\mathcal{B} ; \mathbb{C})}^{2} \leq \frac{1}{3}\left\|\eta_{\mathcal{A}}^{\prime}-\eta_{\mathcal{A}}\right\|_{\ell^{2}(\mathcal{A}, \mathbb{C})}^{2} .
$$

In particular, the map $\kappa: \mathcal{C}_{\mathcal{A}} \rightarrow \mathcal{C}_{\mathcal{B}}$ is continuous.

Proof. Fix an arbitrary element $\eta_{\mathcal{A}} \in \mathcal{C}_{\mathcal{A}}$. Then, by Proposition A.7, for any $\eta_{\mathcal{B}}^{\prime}, \eta_{\mathcal{B}} \in \mathcal{C}_{\mathcal{B}}$

$$
\left\|T\left(\eta_{\mathcal{A}}, \eta_{\mathcal{B}}^{\prime}\right)-T\left(\eta_{\mathcal{A}}, \eta_{\mathcal{B}}\right)\right\|_{\ell^{2}(\mathcal{B} ; \mathbb{C})} \leq \frac{1}{4}\left\|\eta_{\mathcal{B}}^{\prime}-\eta_{\mathcal{B}}\right\|_{\ell^{2}(\mathcal{B} ; \mathbb{C})}
$$


Therefore $T\left(\eta_{\mathcal{A}}, \cdot\right)$ is a contraction map on $\mathcal{C}_{\mathcal{B}}$ and there exists a unique fixed point $\kappa\left(\eta_{\mathcal{A}}\right) \in \mathcal{C}_{\mathcal{B}}$, i.e., $\kappa\left(\eta_{\mathcal{A}}\right)=T\left(\eta_{\mathcal{A}}, \kappa_{\mathcal{B}}\left(\eta_{\mathcal{A}}\right)\right)$.

For $\eta_{\mathcal{A}}, \eta_{\mathcal{A}}^{\prime} \in \mathcal{C}_{\mathcal{A}}$ one obtains, again by Proposition A.7,

$$
\begin{aligned}
\left\|\kappa\left(\eta_{\mathcal{A}}^{\prime}\right)-\kappa\left(\eta_{\mathcal{A}}\right)\right\|^{2} & =\left\|T\left(\eta_{\mathcal{A}}^{\prime}, \kappa\left(\eta_{\mathcal{A}}^{\prime}\right)\right)-T\left(\eta_{\mathcal{A}}, \kappa\left(\eta_{\mathcal{A}}\right)\right)\right\|^{2} \\
& \leq \frac{1}{4}\left(\left\|\eta_{\mathcal{A}}^{\prime}-\eta_{\mathcal{A}}\right\|^{2}+\left\|\kappa\left(\eta_{\mathcal{A}}^{\prime}\right)-\kappa\left(\eta_{\mathcal{A}}\right)\right\|^{2}\right) .
\end{aligned}
$$

As a consequence, as claimed,

$$
\left\|\kappa\left(\eta_{\mathcal{A}}^{\prime}\right)-\kappa\left(\eta_{\mathcal{A}}\right)\right\|^{2} \leq \frac{1}{3}\left\|\eta_{\mathcal{A}}^{\prime}-\eta_{\mathcal{A}}\right\|^{2}
$$

\section{Proof of Theorem 2.1.}

To prove Theorem 2.1 we need one more auxilary result. To formulate it, introduce for a real valued potential $q_{0} \in L_{0}^{2}\left(S^{1}\right)$ circles $\tilde{\Gamma}_{k}\left(q_{0}\right)$ in $\mathbb{C}$ of radius $\frac{1}{2} \gamma_{k}\left(q_{0}\right)+\frac{1}{k} 2 K\left(q_{0}\right)$ and center $\tau_{k}\left(q_{0}\right)$ where $K\left(q_{0}\right):=\frac{1}{5} \min _{n \geq 0}\left(\lambda_{2 n+1}\left(q_{0}\right)-\right.$ $\left.\lambda_{2 n}\left(q_{0}\right)\right)$. Denote by $\tilde{D}_{k}\left(q_{0}\right)$ the closed disk with boundary $\tilde{\Gamma}_{k}\left(q_{0}\right)$.

Lemma A.9. Let $q_{0} \in L_{0}^{2}\left(S^{1} ; \mathbb{C}\right)$ be real valued. Then there exists $N\left(q_{0}\right) \geq$ 1 so that for any $N \geq N\left(q_{0}\right)$ there exists a neighborhood $U$ of $q_{0}$ in $L_{0}^{2}\left(S^{1} ; \mathbb{C}\right)$ with the following properties: For any $q \in U,\left|\lambda_{j}(q)-\lambda_{j}\left(q_{0}\right)\right| \leq \frac{1}{k} K\left(q_{0}\right)$ $(1 \leq k \leq N, j=2 k, 2 k-1)$, and, given arbitrary complex numbers $\mu_{k}(1 \leq$ $k \leq N)$ inside $\tilde{\Gamma}_{k}\left(q_{0}\right)$, the Dirac measures $\left((-1)^{k} 2 \pi^{2} k^{2} \delta_{\mu_{k}}\right)_{1 \leq k \leq N}$ together with $\left(n A_{n}(q)\right)_{n \geq N+1}$ form a Riesz basis of $I_{3 / 2}^{*}$.

Proof. The proof relies on the following three observations:

(1) For any sequence $\left(\mu_{k}\right)_{k \geq 1}$ with $\mu_{k}$ in $\tilde{D}_{k}\left(q_{0}\right)$, the Dirac measures $(-1)^{k} 2 \pi^{2} k^{2} \delta_{\mu_{k}}$ form a Riesz basis of $I_{3 / 2}^{*}$. To see this we first observe that there exists $N_{0} \geq 1$ such that

$$
\sum_{n \geq N_{0}+1}\left\|(-1)^{k} 2 \pi^{2} k^{2} \delta_{\mu_{k}}-(-1)^{k} 2 \pi^{2} k^{2} \delta_{\pi^{2} k^{2}}\right\|_{I_{3 / 2}^{*}}^{2}<\frac{1}{4}
$$

for any choice of $\mu_{k}$ in $\tilde{D}_{k}\left(q_{0}\right)$.

Recall (cf. Remark after Theorem A.3) that $\left((-1)^{k} 2 \pi^{2} k^{2} \delta_{\pi^{2} k^{2}}\right)_{k \geq 1}$ is an orthonormal basis of $I_{3 / 2}^{*}$. Thus $\left((-1)^{k} 2 \pi^{2} k^{2} \delta \mu_{k}\right)_{k \geq N_{0}+1}$ spans a subspace of $I_{3 / 2}^{*}$ of codimension $N_{0}$. By Bari's theorem, adding precisely $N_{0}$ Dirac measures $\left((-1)^{k} 2 \pi^{2} k^{2} \delta \mu_{k}\right)_{1 \leq k \leq N_{0}}$ to this set will give rise to a Riesz basis as soon as $\left((-1)^{k} 2 \pi^{2} k^{2} \delta \mu_{k}\right)_{k \geq 1}$ is $\omega$-linearly independent. To prove the linear 
independence, introduce $\psi_{j}(\lambda):=\frac{1}{j^{2}} \prod_{k \neq j} \frac{\mu_{k}-\lambda}{k^{2} \pi^{2}}$. These functions are elements of $I_{3 / 2}$ and satisfy $\delta_{\mu_{k}}\left(\psi_{j}\right)=0$ for $k \neq j$ and $\delta_{\mu_{j}}\left(\psi_{j}\right) \neq 0$. This proves that $\left((-1)^{k} 2 \pi^{2} k^{2} \delta \mu_{k}\right)_{k \geq 1}$ is $\omega$-linearly independent.

(2) Introduce for any sequence $\mu=\left(\mu_{k}\right)_{k \geq 1}$ with $\mu_{k}$ in $\tilde{D}_{k}\left(q_{0}\right)$ the map $T_{\mu}$ : $I_{3 / 2}^{*} \rightarrow I_{3 / 2}^{*}$ given by $T_{\mu}\left((-1)^{k} 2 \pi^{2} k^{2} \delta_{\pi^{2} k^{2}}\right)=(-1)^{k} 2 \pi^{2} k^{2} \delta_{\mu_{k}}$, corresponding to the change of basis from the orthonormal basis $\left((-1)^{k} 2 \pi^{2} k^{2} \delta_{\pi^{2} k^{2}}\right)_{k \geq 1}$ to the Riesz basis (cf. (1)) $\left((-1)^{k} 2 \pi^{2} k^{2} \delta_{\mu_{k}}\right)_{k \geq 1}$. Then $T_{\mu}$ is a bounded invertible operator, $\left\|T_{\mu}\right\|_{\mathcal{L}\left(I_{3 / 2}^{*}\right)}<\infty$ and $\left\|T_{\mu}^{-1}\right\|_{\mathcal{L}\left(I_{3 / 2}^{*}\right)}<\infty$. One verifies that $T_{\mu}$ depends continuously on $\mu=\left(\mu_{k}\right)_{k \geq 1} \in \prod_{k \geq 1} \tilde{D}_{k}\left(q_{0}\right) \subseteq \ell^{2}(\mathbb{N} ; \mathbb{C})$. Due to the fact that the Hilbert cube $\prod_{k \geq 1} \tilde{D}_{k}\left(q_{0}\right)$ is compact in $\ell^{2}(\mathbb{N} ; \mathbb{C})$ we conclude that there exists $C\left(q_{0}\right)>0$ such that $\left\|T_{\mu}\right\|_{\mathcal{L}\left(I_{3 / 2}^{*}\right)}^{2},\left\|T_{\mu}^{-1}\right\|_{\mathcal{L}_{\left(I_{3 / 2}^{*}\right)}}^{2} \leq C\left(q_{0}\right)$ for all $\mu \in \prod_{k \geq 1} \tilde{D}_{k}\left(q_{0}\right)$.

(3) By standard arguments one shows that there exists $N\left(q_{0}\right) \geq 1$ such that (with $C\left(q_{0}\right)>0$ given as in $(2)$ )

$$
\sum_{k \geq N\left(q_{0}\right)+1}\left\|(-1)^{k} 2 \pi^{2} k^{2} \delta_{\tau_{k}\left(q_{0}\right)}-k A_{k}\left(q_{0}\right)\right\|_{I_{3 / 2}^{*}}^{2} \leq \frac{1}{4} \frac{1}{C\left(q_{0}\right)} .
$$

We now combine (1), (2) and (3) to prove the Lemma A.9:

For $N \geq N\left(q_{0}\right)$ given, choose a neighborhood $U$ of $q_{0}$ in $L_{0}^{2}\left(S^{1} ; \mathbb{C}\right)$ so that for $q$ in $U$ (cf. Corollary A.2)

$$
\sum_{n \geq 1}\left\|n A_{n}(q)-n A_{n}\left(q_{0}\right)\right\|_{I_{3 / 2}^{*}}^{2} \leq \frac{1}{4} \frac{1}{C\left(q_{0}\right)}
$$

and, in addition, for $1 \leq k \leq N$ and $j \in\{2 k, 2 k-1\},\left|\lambda_{j}(q)-\lambda_{j}\left(q_{0}\right)\right| \leq \frac{1}{k} K$ as well as $\left|\lambda_{0}(q)-\lambda_{0}\left(q_{0}\right)\right| \leq K$. Combining these estimates with (A.18) and (A.17) one concludes that, for $q \in U$,

$$
\sum_{n \geq N+1}\left\|n A_{n}(q)-(-1)^{n} 2 n^{2} \pi^{2} \delta_{\tau_{n}\left(q_{0}\right)}\right\|_{I_{3 / 2}^{*}}^{2} \leq \frac{1}{2} \frac{1}{C\left(q_{0}\right)} .
$$

In view of observation (2) and Bari's theorem (cf. [GK, p. 310]) one sees that $\left(\delta_{\mu_{k}}\right)_{1 \leq k \leq N(\epsilon)^{\prime}}\left(n A_{n}(q)\right)_{n \geq N(\epsilon)+1}$ is a Riesz basis for any $q \in U$ and arbitrary $\mu_{k} \in \tilde{D}_{k}\left(q_{0}\right), 1 \leq k \leq N(\epsilon)$.

Proof of Theorem 2.1. For a given real valued potential $q_{0} \in L_{0}^{2}\left(S^{1} ; \mathbb{C}\right)$ let $K_{1}:=K\left(q_{0}\right)$ and choose a neighborhood $U_{1}$ of $q_{0}$ in $L_{0}^{2}\left(S^{1}: \mathbb{C}\right)$ so that the statements of Theorem A.3 hold. Next choose $N \geq 1$ and a neighborhood $U_{2} \subset U_{1}$ of $q_{0}$ in $L_{0}^{2}\left(S^{1} ; \mathbb{C}\right)$ so that the statements of Propositions A.6, A.7, 
Corollary A.8 and Lemma A.9 are valid. For this $N$, let $K_{2}:=\frac{1}{N} K\left(q_{0}\right)$ and choose a neighborhood $U_{3} \subset U_{2}$ of $q_{0}$ in $L_{0}^{2}\left(S^{1} ; \mathbb{C}\right)$ so that the statements of Theorem A.5 hold. In particular, for any $q$ in $U_{3}$ and $j \geq 1$, the function $\frac{1}{j} 1_{j}(\lambda, q)$ has precisely one zero $\mu_{k}^{(j)}(q)$ inside the circle $\Gamma_{k}\left(K_{2}\right)$ for $k$ in $\mathcal{A}_{N j}:=$ $\{1 \leq k \leq N, k \neq j\}$. Let $U_{\mathcal{A}_{N j}}:=\left(\mu_{k}^{(j)}(q)\right)_{k \in \mathcal{A}_{N j}}\left(q \in U_{3}, j \geq 1\right)$ and denote by $\eta_{\mathcal{B}} \equiv \eta_{\mathcal{B}_{N j}}$ the unique fixed point $\kappa\left(\eta_{\mathcal{A}_{N j}}\right)$, provided by Corollary A.8. Define the entire function $\psi_{j}$ in $I_{3 / 2}$ given by

$$
\psi_{j}(\lambda, q):=\frac{1}{j^{2}} \prod_{k \in \mathcal{A}_{N j}} \frac{\mu_{k}^{(j)}(q)-\lambda}{k^{2} \pi^{2}} \prod_{k \in \mathcal{B}_{N j}} \frac{\left(\eta_{\mathcal{B}}\right)_{k}-\lambda}{k^{2} \pi^{2}} .
$$

By construction,

$$
\delta_{\mu_{k}^{(j)}(q)}\left(\psi_{j}(\cdot, q)\right)=0=\delta_{\mu_{k}^{(j)}(q)}\left(\frac{1}{j} 1_{j}(\cdot, q)\right) \quad\left(k \in \mathcal{A}_{N j}\right)
$$

and

$$
A_{k}(q)\left(\psi_{j}(\cdot, q)\right)=0=A_{k}(q)\left(\frac{1}{j} 1_{j}(\cdot, q)\right) \quad\left(k \in \mathcal{B}_{N j}\right) .
$$

For $1 \leq j \leq N$ it follows from Theorem A.5 that $\delta_{\tau_{j}\left(q_{0}\right)}\left(\frac{1}{j} 1_{j}(\cdot, q)\right) \neq 0$ as $\frac{1}{j} 1_{j}(\cdot, q)$ has no zeroes inside $\Gamma_{j}$. By construction $\delta_{\tau_{j}\left(q_{0}\right)}\left(\psi_{j}\right) \neq 0$. Therefore, there exists $C_{j} \equiv C_{j}(q) \neq 0$ such that

$$
\delta_{\tau_{j}\left(q_{0}\right)}\left(C_{j} \psi_{j}\right)=\delta_{\tau_{j}\left(q_{0}\right)}\left(\frac{1}{j} 1_{j}(\cdot, q)\right) .
$$

By Lemma A.9, $\left((-1)^{k} 2 \pi^{2} k^{2} \delta_{\mu_{k}^{(j)}(q)}\right)_{k \in \mathcal{A}_{N j}},(-1)^{j} 2 \pi^{2} j^{2} \delta_{\tau_{j}\left(q_{0}\right)},\left(k A_{k}(q)\right)_{k \geq N+1}$ is a Riesz basis of $I_{3 / 2}^{*}$. This implies that $C_{j} \psi_{j}(\lambda, q)=\frac{1}{j} 1_{j}(\lambda, q)$ for $1 \leq j \leq N$ and $q \in U_{3}$. For $j \geq N+1$, one shows, using standard asymptotic results, that $A_{j}(q)\left(\psi_{j}(\cdot, q)\right) \neq 0$. Recall that $j A_{j}(q)\left(\frac{1}{j} 1_{j}(\cdot, q)\right)=1$ and thus there exists $C_{j} \equiv C_{j}(q) \neq 0$ with $j A_{j}(q)\left(C_{j} \psi_{j}(\cdot, q)\right)=1$.

By Lemma A.9, $\left((-1)^{k} 2 \pi^{2} k^{2} \delta_{\mu_{k}^{(j)}(q)}\right)_{1 \leq k \leq N},\left(\frac{1}{k} A_{k}(q)\right)_{k \geq N+1}$ is a Riesz basis of $I_{3 / 2}^{*}$. This implies that $C_{j} \psi_{j}(\lambda, q)=\frac{1}{j} 1_{j}(\lambda, q)$ for $j \geq N+1$ and $q \in U_{3}$. By definition of the Hilbert cube $\mathcal{C}_{\mathcal{B}}$ (cf. Section A.3) $\left(\mu_{k}^{(j)}(q)-\tau_{k}(q)\right)=: \xi_{k}^{(j)}$ $(k \in \mathcal{B})$ satisfy the estimates stated in Theorem 2.1 and the analyticity of the zeroes $\mu_{k}^{(j)}(q)(k \in \mathcal{B})$ follows again from Cauchy's integral formula

$$
\mu_{k}^{(j)}(q)=\frac{1}{2 \pi i} \int_{\Gamma_{k}} \lambda \frac{\frac{d}{d \lambda} 1_{j}(\lambda, q)}{1_{j}(\lambda, q)} d \lambda
$$




\section{References}

[BBGK] D. Bättig, A. Bloch, J.C. Guillot and T. Kappeler, On the symplectic structure of the phase space for periodic KdV, Toda and defocusing NLS, Duke Math. J., 79 (1995), 549-604.

[BKM] D. Bättig, T. Kappeler and B. Mityagin, On the Korteweg de Vries equation: Convergent Birkhoff normal form, to appear in J.of Funct. Anal.

[Bo1] J. Bourgain, Fourier transform restriction phenomena for certain lattice subsets and applications to nonlinear evolution equations, part II: KdV-equation, GAFA, 3 (1993), 209-262.

[Bo2] J. Bourgain, private communication.

[DKN] B.A. Dubrovnin, I.M. Krichever and S.P. Novikov, in Integrable systems I, Dynamical Systems IV, ed. V.I. Arnold, S.P. Novikov, Encyl. of Math. Sci., Springer Verlag, 1990.

[EM] N. Ercolani and H.P. McKean, Geometry of KdV(4). Abel sums, Jacobi variety and theta function in the scattering case, Invent. Math., 99 (1990), 483-544.

[FKT] J. Feldman, H. Knörrer and E. Trubowitz, Riemann surfaces of infinite genus I. $L^{2}$-cohomology, exhaustions with finite charge and theta series, ETH-preprint.

[FFM] H. Flaschka, M.G. Forest and D. McLaughlin, Multiphase averaging and the inverse spectral solution of the Korteweg-de Vries equation, CPAM, 33 (1980), 739-784.

[GK] I.C. Gohberg and M.G. Krein, Introduction to the theory of linear nonselfadjoint operators, Vol. 18, Translation of Math. Monographs, AMS, 1969.

[Ka] T. Kappeler, Foliation of the phase space for the Korteweg-de Vries equation, Ann. Inst. Fourier, 41 (1991), 539-575.

[Ma] V.A. Marcenko, Sturm Liouville Operators and Applications, Birkhäuser, Basel, 1986.

[MM] H.P. McKean and P. van Moerbeke The spectrum of Hill's operator, Invent. Math., 30 (1975), 217-274.

[MT1] H. P. McKean and E. Trubowitz, Hill's operator and hyperelliptic function theory in the presence of infinitely many branch points, CPAM, 24 (1976), 143-226.

[MT2] _ Hill's surfaces and their theta functions, Bull. A.M.S., 84 (1978), 10421085.

[PT] J. Poschel and E. Trubowitz, Inverse Spectral Theory, Academic Press, 1987.

[Zh] B.Y. Zhang, A remark on the Cauchy problem for the KdV equation on a periodic domain, IMA Preprint, 1993, \#1155.

Received February 26, 1996.

Université PARIS-Nord, France

Ohio State University

Columbus, OH 43210 